\title{
Food intake biomarkers for berries and grapes
}

\author{
M. Ulaszewska ${ }^{1,2+}$ D, M. Garcia-Aloy ${ }^{3,4^{*}+}$, N. Vázquez-Manjarrez ${ }^{5,6,7}$, M. T. Soria-Florido ${ }^{3}$, R. Llorach ${ }^{3,4}$, F. Mattivi $i^{1,8}$ \\ and C. Manach ${ }^{5}$
}

\begin{abstract}
Grapes and berries are two types of widely consumed fruits characterized by a high content in different phytochemicals. However, their accurate dietary assessment is particularly arduous, because of the already wide recognized bias associated with self-reporting methods, combined with the large range of species and cultivars and the fact that these fruits are popularly consumed not only in fresh and frozen forms but also as processed and derived products, including dried and canned fruits, beverages, jams, and jellies. Reporting precise type and/or quantity of grape and berries in FFQ or diaries can obviously be affected by errors. Recently, biomarkers of food intake (BFIs) rose as a promising tool to provide accurate information indicating consumption of certain food items. Protocols for performing systematic reviews in this field, as well as for assessing the validity of candidate BFIs have been developed within the Food Biomarker Alliance (FoodBAll) Project. This paper aims to evaluate the putative BIFs for blueberries, strawberries, raspberries, blackberries, cranberries, blackcurrant, and grapes. Candidate BFIs for grapes were resveratrol metabolites and tartaric acid. The metabolites considered as putative BFI for berries consumption were mostly anthocyanins derivatives together with several metabolites of ellagitannins and some aroma compounds. However, identification of BFls for single berry types encountered more difficulties. In the absence of highly specific metabolites reported to date, we suggested some multi-metabolite panels that may be further investigated as putative biomarkers for some berry fruits.
\end{abstract}

Keywords: Grape, Raisin, Strawberry, Blueberry, Blackberry, Cranberry, Raspberry, Blackcurrant, Biomarkers, Intake

\section{Introduction}

Fruits are important components of a healthy diet [1]. Increased consumption of fruits and vegetables is recommended in dietary guidelines worldwide, and the intake of fruits like berries, which are rich in nutrients, vitamins, minerals, and phytochemicals, may play a role in disease prevention [116]. In terms of production worldwide, according to FAOSTAT (http://www.fao.org/ faostat/en/\# consulted on 28.02.2020), strawberries were

\footnotetext{
* Correspondence: mar.garcia.aloy@gmail.com

${ }^{+}$M. Ulaszewska and M. Garcia-Aloy contributed equally to this work

${ }^{3}$ Biomarkers and Nutrimetabolomic Laboratory, Department of Nutrition,

Food Sciences and Gastronomy, Food Technology Reference Net (XaRTA),

Nutrition and Food Safety Research Institute (INSA-UB), Faculty of Pharmacy

and Food Sciences, University of Barcelona, Barcelona, Spain

${ }^{4}$ CIBER de Fragilidad y Envejecimiento Saludable (CIBERFES), Instituto de

Salud Carlos III, Barcelona, Spain

Full list of author information is available at the end of the article
}

the most intensively produced berry, dominated by China, USA, Mexico, and Turkey with production in kilotonnes (Kt): $2955.4 \mathrm{Kt}, 1296.3 \mathrm{Kt}, 653.6 \mathrm{Kt}$, and 440.9 $\mathrm{Kt}$, respectively. Cranberries were the second in the ranking with USA and Canada producing $404.9 \mathrm{Kt}$ and 195.2 Kt, respectively. Production of raspberries was dominated by Russia, Mexico, Serbia, and Poland with $165.8 \mathrm{Kt}, 130.2 \mathrm{Kt}, 127.0 \mathrm{Kt}$, and $115.6 \mathrm{Kt}$, respectively. The top three producers of grapes worldwide in 2018 were China, Italy and USA with the following kilotonnes: $13,397.0 \mathrm{Kt}, 8513.6 \mathrm{Kt}$, and $6890.9 \mathrm{Kt}$. According to the European food consumption database, northern countries, especially Finland and Latvia but also Spain and Romani are the biggest consumers of berries within Europe, consuming $1.38 \mathrm{~kg} /$ year, $1.24 \mathrm{~kg} /$ year, $1.28 \mathrm{~kg} /$ year, and $1.17 \mathrm{~kg} /$ year. The strawberries $(4.77 \mathrm{~g} /$ day $)$ are

(c) The Author(s). 2020 Open Access This article is licensed under a Creative Commons Attribution 4.0 International License, which permits use, sharing, adaptation, distribution and reproduction in any medium or format, as long as you give appropriate credit to the original author(s) and the source, provide a link to the Creative Commons licence, and indicate if changes were made. The images or other third party material in this article are included in the article's Creative Commons licence, unless indicated otherwise in a credit line to the material. If material is not included in the article's Creative Commons licence and your intended use is not permitted by statutory regulation or exceeds the permitted use, you will need to obtain permission directly from the copyright holder. To view a copy of this licence, visit http://creativecommons.org/licenses/by/4.0/. 
consumed most frequently, followed by blueberries (1.34 g/day) [42]. Berry fruits are popularly consumed not only in fresh and frozen forms but also as processed and derived products, including dried and canned fruits, yogurts, beverages, jams, and jellies, making the evaluation of their intake rather delicate when using questionnaires. On the other hand, grapes, excluding wine, are mainly consumed as fresh fruits, and mean consumption in European countries, according to comprehensive European food consumption database, is $4.93 \mathrm{~g} /$ day [42]. Among them, Hungary and Romania consume the highest amount of grapes $9.7 \mathrm{~g} /$ day and $8.8 \mathrm{~g} /$ day, respectively $[42,180]$.

Berry fruits have attracted considerable attention due to their potential benefits to human health. Botanical classification defines berry as a fruit with seeds produced from the ovary of a single flower. According to this definition category, berries include blueberries, cranberries, lingonberries, Ribes species, grapes, but also bananas, tomatoes or eggplants. On the other hand, strawberries, raspberries, and blackberries are not botanical berries, however enter this category in common nomenclature. This review includes both botanical and commonly called berries: strawberries (Fragaria $\times$ ananassa), raspberries (Rubus idaeus) and blackberries (Rubus fruticosus, other Rubus spp.), blueberries (Vaccinium fruits, i.e, V. myrtillus or V. corymbosum), American cranberries (Vaccinium macrocarpon), and blackcurrant (Ribes nigrum). They provide high levels of antioxidants, vitamins, minerals, and fibres and are amongst the richest sources of phenolic compounds in the human diet $[10,43,120]$. The biological activities of berries have been mainly attributed to their high content of a diverse range of flavonoids (anthocyanins, flavonols, and flavanols), tannins (proanthocyanidins, ellagitannins, and gallotannins), phenolic acids (hydroxybenzoic and hydroxycinnamic acid derivatives), and lignans. In particular, cranberries are known for their effect on urinary tract infections, while blueberries (a proanthocyanidin-rich fruit) and strawberries (an ellagitannin-rich fruit) are actively studied for their impact on neuronal function and behavior [8, 152, 154, 159].

Grape also contains a wide variety of polyphenols with demonstrated cardioprotective properties. Their polyphenolic content is higher than in other fruits, in the range of $50-490 \mathrm{mg} / 100 \mathrm{~g}$ of fresh matter [18]. However, an important percentage of these are contained in the seeds [102]. Several studies have been focused on the polyphenolic composition of this food revealing its complexity, mainly consisting of proanthocyanidins, anthocyanins, stilbenes, flavan-3ols, and phenolic acids and complex condensation structures [21, 170]. It has been demonstrated that polyphenolic compounds from red grapes could improve endothelial function in patients with coronary heart disease [93], age-related cognitive and motor function [90], or decreases in oxidative stress and inflammatory markers [24, 118]. Considering both the health benefits of grape consumption [180] and the metabolic fate of their components, it became interesting to be able to monitor its intake with an accurate measurement tool.

This work aimed to provide an overview of the metabolites found in biological fluids that could potentially act as BFIs for grape and different berry types, further evaluating their validity considering the current available information and identifying the aspects that require further investigation. There are several examples where a single metabolite could be a biomarker of a particular food intake, such as phloretin for apple intake [166]. However, the composition of each plant food is very complex (from the quantitative and qualitative point of view) and many of the compounds are widely distributed among a variety of foods [62]. Several polyphenols can be considered semi-ubiquitary, they are present in a wide range of plant foods, e.g. chlorogenic acids (in coffee and apple and other fruits) or flavan-3-ols (in cocoa, tea fruit, and wine), and thus, using a single-biomarker strategy is very often not appropriate. On the other hand, the intake of different foods may give rise to similar metabolites in biofluids losing their specificity for a certain dietary source [57]. The ellagitannins and procyanidins represent such cases. Ellagitannins are present in foods such as walnuts, strawberries, Rubus, and pomegranate and are largely metabolized by the microbiota, producing urolithin derivatives. Finally, urolithins indicate the intake of a food containing ellagitannins, but are not specific for any food. Similarly, procyanidins, metabolized by the gut microbiota produce a wide range of valerolactones and valeric acids, which have been proposed as biomarkers of procyanidin-rich foods-tea, cocoa, apple, walnuts, or wine. Considering those examples, the usefulness of these polyphenol metabolites as single biomarkers for dietary intake appears limited. For this reason, a multi-metabolite biomarker panel (MBP) was recently proposed as a tool to capture dietary exposure and improve the accuracy and precision of dietary assessment [62]. This concept has been successfully applied in a number of studies relating the urine metabolite profile to the intake of walnuts [59], cocoa [60], and bread [61]. The results from the aforementioned studies showed that MBP performs better than unique compounds in terms of predicting dietary exposure. The present review thus considered single metabolites acting as BFI, as well as the possibility of using multiple metabolite panels for assessing the intake of berries and grape. 
This review has been performed in the frame of the FoodBAll project (Food Biomarkers Alliance, http:// www.foodmetabolome.org/) funded by the Joint Programming Initiative "A Healthy Diet for a Healthy Life" (JPI-HDHL).

\section{Methods}

\section{Selection of food groups}

For this review, grapes (Vitis vinifera) together with raisins (i.e., dried grapes) were selected as widely consumed members of the Vitaceae family. The second family of fruits included in this work was botanical berries: blueberries, cranberries, blackcurrant and redcurrant, and commonly called berries: strawberries and Rubus fruits (raspberries and blackberries). The selection of berry types was based on the frequency of consumption reported in the FoodEx database [42] and in the literature [110]. The chokeberries and goji berries, as consumed less frequently, were not included in this review.

\section{Search for relevant FIB research papers}

An extensive literature search was carried out to collect all available information on the existing and new candidate BFIs for the selected fruits. The FIBRev protocol (Food Intake Biomarker Reviews) elaborated with guidance of the PRISMA statement (Preferred Reporting Items for Systematic Reviews and Meta-Analyses) and described in Pratico et al. [128] was followed. Briefly, a primary search was performed in the three databases Scopus, PubMed central, and Web of Science to identify compounds that are already used as or may represent potential BFIs. Search for each berry type was performed independently. The name of the specific fruit and its botanical genus, i.e (strawberr* OR fragaria), (blackberr* OR rubus), (raspberr* OR rubus), (redcurrant OR ribes), (blackcurrant OR ribes), (blueberr* OR vaccinium), (cranberr* OR vaccinium), and (grape* OR rasin*) along with the common keywords: AND (urine or plasma or serum or excretion or blood) AND (human* OR men OR women OR patient* OR volunteer* OR participant*) AND (biomarker* OR marker* OR metabolite* OR biokinetics OR biotransformation OR pharmacokinetics OR bioavailability OR ADME) AND (Intake OR meal OR diet OR ingestion OR administration OR consumption OR eating OR drink*) were applied. Keywords were used in the fields [Topic], [All fields], and [Article Title/Abstract/Keywords] for Web of Science, PubMed, and Scopus, respectively. All searches were carried out in March 2016 and updated in December 2019. Only papers in English language were considered eligible, and no restriction on the date of publication was applied. Articles showing results of human intervention studies (randomized controlled trials, acute, short-term or long-term studies) or observational studies (cohort, case-control, cross-sectional studies) were considered eligible. A first selection of papers was performed according to abstract and title relevance. Full texts were obtained for the selected articles and further assessed for eligibility according to their relevance in determining BFIs for grapes and berry fruits. Some of the publications found in the reference list of the selected articles were also included at this stage. The main exclusion criteria were papers that discussed the health effects and in-vitro studies. The research papers identifying or using potential biomarkers of intake were selected according to the process outlined in Fig. 1.

\section{Identification and characterization of candidate BFIs}

A secondary search allowed to retrieve relevant information to assess the quality of every individual candidate biomarker, regarding specificity, biokinetics, dose-response relationship, robustness, and reliability of the method of analysis, in order to validate its use as BFI according to the scoring validating scheme established by Dragsted et al. [40].

The name of the candidate biomarkers and their synonyms were queried in the previously mentioned databases along with AND (biomarker* OR marker* OR metabolite* OR biokinetics OR biotransformation OR pharmacokinetics OR bioavailability OR ADME). Additionally, the compounds were searched manually in the online databases HMDB (https://www.hmdb.ca), FooDB (http://foodb.ca/), Phenol-Explorer (http://phenol-explorer.eu/), Dictionary of Food Compounds (http://dfc. chemnetbase.com/faces/chemical/ChemicalSearch.

xhtml), Duke's phytochemical and ethnobotanical databases (https://phytochem.nal.usda.gov/phytochem/ search), eBASIS (http://ebasis.eurofir.org/Default.asp), Knapsack (http://kanaya.naist.jp/knapsack_jsp/top.html), and PhytoHub (http://phytohub.eu) to determine all the possible dietary or metabolic origins of the candidate BFIs.

Specific and non-specific metabolites were discussed in the text, while only the most plausible candidate BFIs have been reported in Table 1 including the information related to study designs and analytical methods. The non-retained compounds are listed in Table 2, with main reasons for exclusion and references for an exhaustive presentation of the results. The tables have been reviewed and agreed upon by all authors.

\section{Application of validation criteria}

According to Dragsted et al. [40], a set of criteria was applied on the candidate BFIs reported in Table 1, to assess their current status of validation and identify the missing information for a full validation of each of them. 


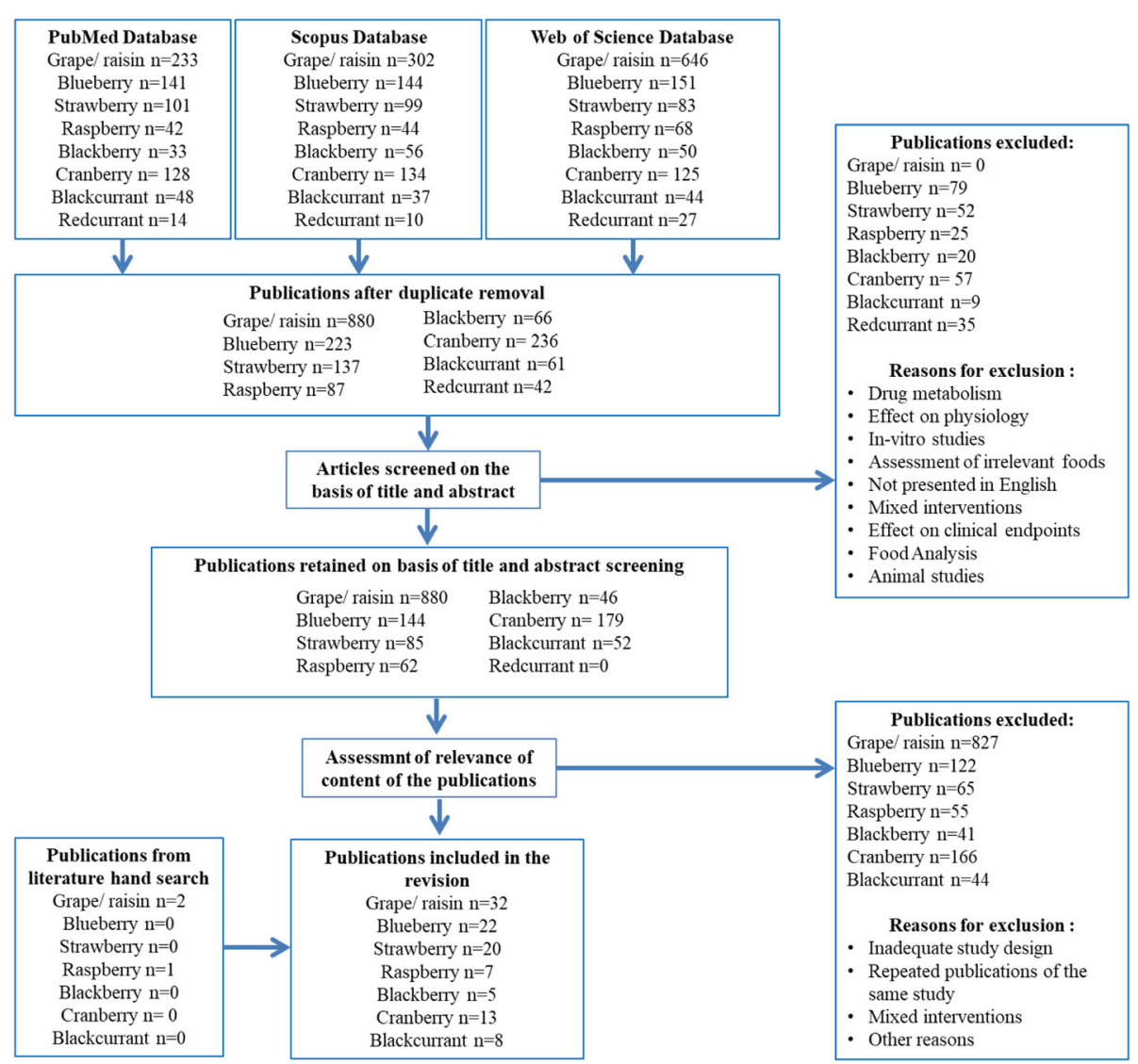

Fig. 1 Flow diagram of study selection according to the BFIRev procedure

\section{Results}

\section{Berry biomarkers}

From 852 citations, 75 human studies were selected for this review (Fig. 1), most of them employing a targeted analysis, while only 7 used an untargeted approach $[2,5$, $7,31,64,94,95]$. Targeted analyses have mainly quantified anthocyanins in their native glycosidic or aglycone forms, as well as ellagitannins and their microbial metabolites. Some investigations also included quantification of phenolic acids and their conjugates.

After careful application of the inclusion and exclusion criteria to the citations collected with the databases search, the numbers of human studies selected for this review were 22 studies out of 206 for blueberry intake $[2,3,12,13,32,34,37,50,81-83,91,103,105,106$, $112,137,153,160,178,185]$; 20 out of 137 for strawberries $[4,5,9,14,20,22,26,31,41,48,74,75,113$, $126,129,141,147,148,165,179]$, 7 out of 87 for raspberry [70, 78, 86, 95, 98, 156, 184], 5 out of 66 for blackberries [47, 64, 89, 100, 161], 13 out of 236 for cranberries $[7,27,49,51,94,108,119,127,135,174-$ $176,183]$, and 8 out of 61 for blackcurrant [44, 76, 80, $105,114,115,139,164]$. No publication on redcurrant could be found relevant for this review.

\section{Grape biomarkers}

From the 880 unique citations screened, 32 original papers fulfilled the inclusion criteria of this review (Fig. 1) $[6,15,19,20,23,24,29,33,55,66,69,71,79,84,85,96$, $97,99,107,117,118,121,122,131,138,142,143,149$, $150,157,158,168,171,177]$. The grape extract was included in this review, considering that elimination of water fraction during desiccation does not drastically change the overall polyphenol content. Most of these studies were nutritional intervention studies with grape juice or grape extract and used a targeted approach to analyze specific metabolites associated with their intake in urine and/or plasma samples. Only few studies used an untargeted metabolomics approach to obtain a holistic view of the metabolites associated with grape intake.

\section{Discussion}

\section{Berries}

Berries, both botanical and aggregate, share several polyphenol families and indeed most of the investigations reported in this review focused on anthocyanins, procyanidins, and other small phenolic metabolites as the main compounds recovered in biological fluids after berry intake. Certain attention was also put on berry 
Table 1 List of studies reporting candidate biomarkers for grape/raisin and berry consumption

\begin{tabular}{|c|c|c|c|c|c|c|}
\hline Dietary factor & Study design & $\begin{array}{l}\text { Number of } \\
\text { subjects }\end{array}$ & $\begin{array}{l}\text { Analytical } \\
\text { method }\end{array}$ & Sample type & $\begin{array}{l}\text { Discriminating } \\
\text { metabolites/candidate } \\
\text { biomarkers }\end{array}$ & Reference(s) \\
\hline \multicolumn{7}{|c|}{ Grapes and raisins } \\
\hline \multirow[t]{2}{*}{ Red grapes (50-200 g) } & \multirow{2}{*}{$\begin{array}{l}\text { Single-dose study, } \\
\text { short-term controlled } \\
\text { study ( } 4 \text { days), } \\
\text { crossover design }\end{array}$} & $6(3 F, 3 M)$ & NMR & $\begin{array}{l}\text { Urine }(0-24 \mathrm{~h} \\
\text { after intake) }\end{array}$ & \multirow[t]{2}{*}{ - Tartaric acid } & \multirow[t]{2}{*}[66]{} \\
\hline & & $19(9 \mathrm{~F}, 10 \mathrm{M})$ & NMR & $\begin{array}{l}\text { Urine (fasting \& } \\
24-h \text { urine) }\end{array}$ & & \\
\hline $\begin{array}{l}\text { Grape juice (200- } \\
1200 \mathrm{~mL})\end{array}$ & Single dose study & $1 \mathrm{M}$ & LC-MS & $\begin{array}{l}\text { Urine (0-4 h after } \\
\text { intake) }\end{array}$ & - Resveratrol & [107] \\
\hline Grape juice (12-18 oz) & Intervention study & $2 M$ & NMR & Urine (overnight) & - Tartaric acid & [96] \\
\hline Grape juice (28 mL) & Intervention study & 23 (not specified) & LC-MS & Urine (overnight) & - Tartaric acid & [97] \\
\hline Red grape juice (1 L) & $\begin{array}{l}\text { Single-dose study, } \\
\text { crossover design }\end{array}$ & $11 \mathrm{M}$ & GC-MS & $\begin{array}{l}\text { Plasma (0-24 h } \\
\text { after intake) } \\
\text { Urine (0-24 h } \\
\text { after intake) }\end{array}$ & $\begin{array}{l}\text { - cis-Resveratrol } \\
\text { - trans-Resveratrol }\end{array}$ & [121] \\
\hline $\begin{array}{l}\text { Red grape juice } \\
\text { (Concord) }(350 \mathrm{~mL} \text { ) }\end{array}$ & Single-dose study & $12(7 F, 5 M)$ & GC-MS & $\begin{array}{l}\text { Urine }(0-24 \mathrm{~h} \\
\text { after intake) }\end{array}$ & - Tartaric acid & {$[158]$} \\
\hline $\begin{array}{l}\text { Grape pomace } \\
\text { beverage }(500 \mathrm{~mL})\end{array}$ & $\begin{array}{l}\text { Crossover, placebo- } \\
\text { controlled }\end{array}$ & $12(6 \mathrm{~F}, 6 \mathrm{M})$ & LC-MS & Urine & $\begin{array}{l}\text { - cis-Resveratrol-3-O- } \\
\text { glucuronide, } \\
\text { - cis-Resveratrol-3-O-sulfate, } \\
\text { - cis-Resveratrol-4"-O- } \\
\text { glucuronide, } \\
\text { - cis-Resveratrol- 4-O-sulfate } \\
\text { - trans-Resveratrol-3-O- } \\
\text { glucuronide, } \\
\text { - trans-Resveratrol-3-O-sulfate, } \\
\text { - trans-Resveratrol-4"-O- } \\
\text { glucuronide, } \\
\text { - trans-Resveratrol-4-O-sulfate } \\
\text { - Dihydroresveratrol-3-O- } \\
\text { sulfate, } \\
\text { - Dihydroresveratrol-4-O- } \\
\text { sulfate, } \\
\text { - Dihydroresveratrol- } \\
\text { sulfoglucuronide }\end{array}$ & [150] \\
\hline $\begin{array}{l}\text { Grape skin extract } \\
\text { beverage (187 mL) }\end{array}$ & $\begin{array}{l}\text { Single dose \& long-term } \\
\text { study ( } 2 \text { weeks), cross } \\
\text { over, placebo-controlled }\end{array}$ & $26(13 \mathrm{~F}, 13 \mathrm{M})$ & UPLC MS/MS & $\begin{array}{l}\text { Urine: } 0-4 \mathrm{~h} \text { after intake } \\
\text { (acute study) and } 24-\mathrm{h} \\
\text { urine (long-term study) }\end{array}$ & $\begin{array}{l}\text { - trans-Resveratrol-3-O- } \\
\text { glucuronide, } \\
\text { - trans-Resveratrol-4'-O- } \\
\text { glucuronide, } \\
\text { - trans-Resveratrol-3'-O- } \\
\text { sulfate, } \\
\text { - trans-Resveratrol-4'-O- } \\
\text { sulfate, } \\
\text { - trans-Resveratrol-3,4'-O- } \\
\text { disulfate } \\
\text { - cis-Resveratrol-3-O- } \\
\text { glucuronide, } \\
\text { - cis-Resveratrol-4'-O- } \\
\text { glucuronide, } \\
\text { - cis-Resveratrol-3'-O-sulfate, } \\
\text { - cis-Resveratrol-4'-O-sulfate } \\
\text { - Resveratrol sulfoglucuronide } \\
\text { - trans and cis-piceid } \\
\text { - Piceid glucuronide, } \\
\text { - Piceid sulfate } \\
\text { - Dihydroresveratrol-3-O- } \\
\text { sulfate, } \\
\text { - Dihydroresveratrol-4-O- } \\
\text { sulfate, } \\
\text { - Dihydroresveratrol- } \\
\text { sulfoglucuronide }\end{array}$ & [143] \\
\hline $\begin{array}{l}\text { Grape extract tablets } \\
\text { (15 tablets with } \\
400 \mathrm{~mL} \text { of water) }\end{array}$ & $\begin{array}{l}\text { Single-dose study, } \\
\text { parallel design }\end{array}$ & $3 \mathrm{M}$ & $\begin{array}{l}\text { LC-ESI-MS/ } \\
\text { MS }\end{array}$ & $\begin{array}{l}\text { Plasma (0-48 h } \\
\text { after intake) } \\
\text { Urine ( } 0-48 \mathrm{~h} \\
\text { after intake) }\end{array}$ & $\begin{array}{l}\text { - trans-Resveratrol-3-O- } \\
\text { glucuronide }(u, p), \\
\text { - trans-Resveratrol-4'-O- } \\
\text { glucuronide }(u, p), \\
\text { - trans-Resveratrol-3-O-sulfate } \\
\text { (u), } \\
\text { - trans-Resveratrol-4'-O-sulfate } \\
\text { (u) } \\
\text { - cis-Resveratrol-3-O- } \\
\text { glucuronide }(u, p),\end{array}$ & {$[142]$} \\
\hline
\end{tabular}


Table 1 List of studies reporting candidate biomarkers for grape/raisin and berry consumption (Continued)

\begin{tabular}{|c|c|c|c|c|c|c|}
\hline Dietary factor & Study design & $\begin{array}{l}\text { Number of } \\
\text { subjects }\end{array}$ & $\begin{array}{l}\text { Analytical } \\
\text { method }\end{array}$ & Sample type & $\begin{array}{l}\text { Discriminating } \\
\text { metabolites/candidate } \\
\text { biomarkers }\end{array}$ & Reference(s) \\
\hline & & & & & $\begin{array}{l}\text { - cis-Resveratrol-4'-O- } \\
\text { glucuronide (u, p), } \\
\text { - cis-Resveratrol-3-O-sulfate } \\
\text { (u), } \\
\text { - cis-Resveratrol-4'-O-sulfate } \\
\text { (u) } \\
\text { - trans- and cis-piceid (u) } \\
\text { - piceid-glucuronide (u, p), } \\
\text { - piceid sulfate (u) } \\
\text { - Dihydroresveratrol- } \\
\text { glucuronide (u, p), } \\
\text { - Dihydroresveratrol- } \\
\text { sulfate (u) }\end{array}$ & \\
\hline $\begin{array}{l}\text { Resveratrol }(250 \mathrm{mg}) \text {, } \\
\text { grape seed extract } \\
(900 \mathrm{mg}) \text { vs grape } \\
\text { juice }(20 \mathrm{oz})\end{array}$ & $\begin{array}{l}\text { Pharmacokinetic } \\
\text { challenge, } 10 \text { days with } \\
\text { repeated doses }\end{array}$ & $7 \mathrm{M}, 5 \mathrm{~F}$ & LC-MSMS & $\begin{array}{l}\text { Blood: } 0.5 \mathrm{~h}, 1 \mathrm{~h}, 1.5 \mathrm{~h}, 2 \\
\mathrm{~h}, 2.5 \mathrm{~h}, 3 \mathrm{~h}, 4 \mathrm{~h}, 5 \mathrm{~h}, 6 \mathrm{~h}\end{array}$ & • Resveratrol & [117] \\
\hline \multicolumn{7}{|l|}{ Blueberry } \\
\hline $\begin{array}{l}\text { Bluberry extract } 25 \mathrm{~g} \text {, } \\
\text { single-dose kinetic } \\
\text { study }\end{array}$ & Single-dose study & 10 & $\begin{array}{l}\text { LC-ESI-MS- } \\
\text { Orbitrap } \\
\text { Untargeted } \\
\text { study }\end{array}$ & $\begin{array}{l}\text { Serum: } 30,60,120, \\
\text { 240, and } 360 \mathrm{~min} \\
\text { Urine: } 30,60,120,240 \\
\text { and } 360 \mathrm{~min}\end{array}$ & $\begin{array}{l}\text { - Delphinidin hexoside }(\mathrm{u})^{*} \\
\text { - Cyanidin hexoside }(\mathrm{u})^{*}\end{array}$ & [2] \\
\hline $\begin{array}{l}300 \mathrm{~g} \text { of blanched vs } \\
\text { unblanched blueberries }\end{array}$ & $\begin{array}{l}\text { Randomized } \\
\text { crossover design }\end{array}$ & $10 \mathrm{M}$ & HPLC-UV & $\begin{array}{l}\text { Blood plasma: } 0 \mathrm{~h}, 1 \mathrm{~h} . \\
2 \mathrm{~h}, 24 \mathrm{~h}\end{array}$ & $\begin{array}{l}\text { - Malvidin-3-glucoside (p) } \\
\text { - Cyanidin-3-glucoside }(p) \\
\text { - Phenyl-y-valerolactone } \\
\text { metabolite }\end{array}$ & [37] \\
\hline Blueberry $250 \mathrm{~mL}$ juice & Single-dose kinetic study & $17(13 \mathrm{~F}, 4 \mathrm{M})$ & $\begin{array}{l}\text { HPLC-ESI-MS/ } \\
\text { MS }\end{array}$ & $\begin{array}{l}\text { Urine: } 0 \mathrm{~h} \text {, every void } \\
\text { during } 24 \mathrm{~h}\end{array}$ & $\begin{array}{l}\text { - Cyanidin metabolites } \\
\text { (including glucuronide and } \\
\text { methyl glucuronide) } \\
\text { - Pelargonidin metabolites } \\
\text { - Delphinidin (including } \\
\text { glucuronide and methyl } \\
\text { glucuronide) } \\
\text { - Malvidin (including } \\
\text { glucuronide and methyl } \\
\text { glucuronide) } \\
\text { - Peonidine (including } \\
\text { glucuronide and methyl } \\
\text { glucuronide) } \\
\text { - Petunidin (including } \\
\text { glucuronide and methyl } \\
\text { glucuronide) }\end{array}$ & [81] \\
\hline $\begin{array}{l}250 \mathrm{~mL} \text { of blueberry } \\
\text { juice per day, for } 28 \\
\text { days ( } 216 \mathrm{mg}(448 \\
\mu \mathrm{mol}) \text { cyn 3-glucoside) }\end{array}$ & $\begin{array}{l}\text { Randomized one-arm } \\
\text { study }\end{array}$ & $17(13 \mathrm{~F}, 4 \mathrm{M})$ & $\begin{array}{l}\text { HPLC-ESI-MS/ } \\
\quad \text { MS }\end{array}$ & $\begin{array}{l}\text { Urine } 24 \mathrm{~h} \text { : days } 0,7 \text { th, } \\
14 \text { th, } 28 \text { th, and } 36 \text { th }\end{array}$ & $\begin{array}{l}\text { - Cyanidin glucuronide } \\
\text { - Delphinidin glucuronide } \\
\text { - Malvidin arabinose } \\
\text { - Malvidin glucuronide } \\
\text { - Malvidin glycoside } \\
\text { - Malvidin methyl } \\
\text { glucuronide } \\
\text { - Pelargonidin glucuronide } \\
\text { - Pelargonidin methyl } \\
\text { glucuronide } \\
\text { - Pelargonidin methyl } \\
\text { glycoside } \\
\text { - Peonidin glucuronide } \\
\text { - Peonidin glycoside } \\
\text { - Peonidin methyl } \\
\text { glucuronide } \\
\text { - Petunidin } \\
\text { - Petunidin glucuronide } \\
\text { - Petunidin methyl } \\
\text { glucuronide }\end{array}$ & [83] \\
\hline $\begin{array}{l}\text { Blueberry powder in } \\
\text { combination with } \\
\text { placebo oil and fish }\end{array}$ & $\begin{array}{l}\text { Randomized, double } \\
\text { blind, parallel groups, } \\
\text { placebo-controlled trial }\end{array}$ & 76 & HPLC-MS & $\begin{array}{l}\text { Urine, morning samples, } \\
\text { after overnight fasting, } \\
\text { day } 0 \text {, day } 12 \text { th }\end{array}$ & $\begin{array}{l}\text { - Total antocyanins including } \\
\text { glucuronide conjugates }\end{array}$ & [106] \\
\hline
\end{tabular}


Table 1 List of studies reporting candidate biomarkers for grape/raisin and berry consumption (Continued)

\begin{tabular}{|c|c|c|c|c|c|c|}
\hline Dietary factor & Study design & $\begin{array}{l}\text { Number of } \\
\text { subjects }\end{array}$ & $\begin{array}{l}\text { Analytical } \\
\text { method }\end{array}$ & Sample type & $\begin{array}{l}\text { Discriminating } \\
\text { metabolites/candidate } \\
\text { biomarkers }\end{array}$ & Reference(s) \\
\hline $\begin{array}{l}\text { blueberry } 100 \mathrm{~g} \\
\text { (containing } 1.2 \mathrm{~g} \\
\text { antocyanins) }\end{array}$ & $\begin{array}{l}\text { Single-blind crossover } \\
\text { with control meal }\end{array}$ & 5 (not specified) & HPLC-UV & $\begin{array}{l}\text { Blood serum: } 0 \mathrm{~h}, 1 \mathrm{~h}, \\
2 \mathrm{~h}, 3 \mathrm{~h}, 4 \mathrm{~h}\end{array}$ & $\begin{array}{l}\text { - Cyanidin-3-glycosides } \\
\text { - Delphinidin-3-glycosides } \\
\text { - Malvidin-3-glycosides } \\
\text { - Petunidin-3-glycosides }\end{array}$ & [103] \\
\hline $\begin{array}{l}300 \mathrm{~mL} \text { blueberry } \\
\text { extract }\end{array}$ & Single-dose crossover & $5 \mathrm{M}$ & HPLC-UV & $\begin{array}{l}\text { Urine: } 0 \mathrm{~h}, 60 \mathrm{~min}, 120 \\
\mathrm{~min}, 180 \mathrm{~min}, 240 \mathrm{~min}, \\
300 \mathrm{~min}\end{array}$ & $\begin{array}{l}\text { - Cyanidin-3-glycosides } \\
\text { - Delphinidin-3-glycosides } \\
\text { - Malvidin-3-glycosides } \\
\text { - Petunidin-3-glycosides }\end{array}$ & {$[105]$} \\
\hline $10 \mathrm{~g}$ bilberry extract & Single dose & $10 \mathrm{~F}$ & HPLC-MS/MS & $\begin{array}{l}\text { Urine: } 0-2 h, 2-4 h, 4-8 h \text {, } \\
\text { Blood: } 0-2 h, 2-4 h \text {, } \\
4-8 h, 8-24 h \\
\text { Ileostomy fluid: } 0-1 h, 1- \\
2 h, 2-4 h, 4-6 h, 6-8 h\end{array}$ & $\begin{array}{l}\text { - Malvidin glucuronide } \\
\text { - Peonidin glucuronide }\end{array}$ & [112] \\
\hline $\begin{array}{l}\text { 189g frozen blueberry } \\
\text { blended with } 315 \mathrm{ml} \\
\text { water }\end{array}$ & $\begin{array}{l}\text { Parallel randomized } \\
\text { study }\end{array}$ & $5 \mathrm{~F}$ & HPLC-DAD & $\begin{array}{l}\text { Blood plasma } 10 \mathrm{~min}, 20 \\
\mathrm{~min}, 30 \mathrm{~min}, 45 \mathrm{~min}, 1 \mathrm{~h} \text {, } \\
2 \mathrm{~h}, 4 \mathrm{~h}, 6 \mathrm{~h}, \text { and } 24 \mathrm{~h} \\
\text { postprandial } \\
\text { Urine: } 0 \mathrm{~h}, 0-2 \mathrm{~h}, 2-4 \mathrm{~h} \text {, } \\
4-6 \mathrm{~h}, 6-8 \mathrm{~h}, 8-12 \mathrm{~h}, 12- \\
24 \mathrm{~h}\end{array}$ & $\begin{array}{l}\text { - Cyanidin-3-glycosides } \\
\text { - Cyanidin-3-glucoside } \\
\text { monoglucuronide } \\
\text { - Delphinidin-3-glycosides } \\
\text { - Malvidin-3-glycosides } \\
\text { - Petunidin-3-glycosides }\end{array}$ & {$[178]$} \\
\hline $\begin{array}{l}25 \mathrm{~g} \text { freeze dried wild } \\
\text { blueberry powder }\end{array}$ & $\begin{array}{l}\text { Single blind, randomized, } \\
\text { two-arm crossover- } \\
\text { controlled study }\end{array}$ & $6 \mathrm{M}, 6 \mathrm{~F}$ & $\begin{array}{l}\text { UHPLC-Q- } \\
\text { TOF-MS'; } \\
\text { UHPLC-QQQ- } \\
\text { MS }^{2}\end{array}$ & $\begin{array}{l}\text { Blood: } 0.25 \mathrm{~h}, 0.5 \mathrm{~h}, 1 \mathrm{~h}, \\
2 \mathrm{~h}, 4 \mathrm{~h}, 6 \mathrm{~h}, 8 \mathrm{~h}, 10 \mathrm{~h}, \\
24 \mathrm{~h} \text { fasting }\end{array}$ & $\begin{array}{l}\text { - Malvidin-3-(6"-acetyl- } \\
\text { glucoside) } \\
\text { - Chlorogenic acid } \\
\text { - Cyanidin 3-arabinose } \\
\text { - Cyanidin-3-galactoside } \\
\text { - Cyanidin-3-glucoside } \\
\text { - Cyanidin glucuronide } \\
\text { - Delphinidin-3-arabinose } \\
\text { - Delphinidin-3-galactoside } \\
\text { - Delphinidin-3-glucoside } \\
\text { - Delphinidin glucuronide } \\
\text { - Malvidin-3-arabinose } \\
\text { - Malvidin-3-galactoside } \\
\text { - Malvidin-3-glucoside } \\
\text { - Peonidin-3-arabinose } \\
\text { - Peonidin-3-galactoside } \\
\text { - Peonidin-3-glucoside } \\
\text { - Peonidin glucuronide } \\
\text { - Petunidin-3-arabinose } \\
\text { - Petunidin-3-galactoside } \\
\text { - Petunidin-3-glucoside } \\
\text { - Petunidin glucuronide }\end{array}$ & {$[185]$} \\
\hline \multicolumn{7}{|l|}{ Strawberry } \\
\hline $\begin{array}{l}300 \mathrm{~g} \text { Fresh strawberries } \\
(\mathrm{fs}) \text { and stored } \\
\text { strawberries (ss) }\end{array}$ & Single-dose,crossover & 13 (not specified) & LC-MS/MS & $\begin{array}{l}\text { Blood plasma: } 0.5,1,2,3 \text {, } \\
5, \text { and } 8 \mathrm{~h} \\
\text { Urine: } 0-2,2-4,4-6,6-8 \text {, } \\
8-12 \text {, and } 12-24 \mathrm{~h}\end{array}$ & $\begin{array}{l}\text { - Pelargonidin (u) } \\
\text { - Pelargonidin glucoside (u) } \\
\text { - Pelargonidin glucuronide } \\
\text { (u) }\end{array}$ & {$[4] ;$} \\
\hline $\begin{array}{l}\text { Strawberry powder: } \\
10 \mathrm{~g}, 20 \mathrm{~g}, 40 \mathrm{~g}\end{array}$ & $\begin{array}{l}\text { Single-center, } \\
\text { randomized, single-blind, } \\
\text { four-arm, crossover }\end{array}$ & 5 (not specified) & $\begin{array}{c}\text { Q-TOF LC/MS } \\
\text { and LC-MS/ } \\
\text { MS }\end{array}$ & $\begin{array}{l}\text { Blood plasma: } 0 \mathrm{~h}, 30 \\
\mathrm{~min}, 60 \mathrm{~min}, 90 \mathrm{~min}, 120 \\
\mathrm{~min}, 180 \mathrm{~min}, 240 \mathrm{~min}, \\
300 \mathrm{~min}, 360 \mathrm{~min}\end{array}$ & $\begin{array}{l}\text { - Pelargonidin-O-glucuronide } \\
\text { - Pelargonidin sulfate } \\
\text { - Pelargonidin glycosides } \\
\text { - Cyanidin glycosides }\end{array}$ & {$[5]$} \\
\hline $\begin{array}{l}50 \mathrm{~g} \text { Daily of freeze- } \\
\text { dried strawberry } \\
\text { powder for } 4 \text { weeks }\end{array}$ & 4-week one-arm study & $16 \mathrm{~F}$ & HPLC-UV & $\begin{array}{l}\text { Fasting serum and } \\
\text { plasma }\end{array}$ & • Ellagic acid & {$[9]$} \\
\hline $\begin{array}{l}500 \mathrm{~g} \text { Strawberries: } \\
\text { integrated food } \\
\text { production vs } \\
\text { organic production }\end{array}$ & $\begin{array}{l}\text { Open-label crossover } \\
\text { design with run-in and } \\
\text { washout periods and } \\
\text { consisted of } 8 \text { visits at } \\
\text { the 0th, } 10 \text { th, } 25 \text { th, } 40 \text { th, } \\
50 \text { th, } 65 \text { th, 80th, and } \\
\text { 90th day of observation }\end{array}$ & $33(16 \mathrm{M} ; 17 \mathrm{~F})$ & $\begin{array}{l}\text { HPLC-UVNIS, } \\
\text { HPLC-ECD }\end{array}$ & $\begin{array}{l}\text { Fasting plasma and } \\
\text { spot morning urine }\end{array}$ & - Urolithin A & {$[14]$} \\
\hline $\begin{array}{l}\text { Strawberry juice, } 55 \mathrm{~g} \\
\text { of fruits }\end{array}$ & Single-intake study & $3 \mathrm{M}, 3 \mathrm{~F}$ & HPLC-MS & $\begin{array}{l}\text { Urine: } 2 \mathrm{~h}, 4 \mathrm{~h}, 6 \mathrm{~h} \text {, } \\
8 \mathrm{~h} \text {, and } 24 \mathrm{~h}\end{array}$ & $\begin{array}{l}\text { - Pelargonidin-3-glucoside } \\
\text { - Pelargonidin-3-rutinoside } \\
\text { - Pelargonidin glucuronide }\end{array}$ & [20] \\
\hline $\begin{array}{l}\text { Strawberry puree } \\
100 \mathrm{~g}, 200 \mathrm{~g}, 400 \mathrm{~g}\end{array}$ & $\begin{array}{l}\text { Dose-response crossover } \\
\text { study dosing }\end{array}$ & 12 (not specified) & HPLC-UV-MS & $\begin{array}{l}\text { Urine: } 0,2,4,6,8,10 \text {, } \\
12 \text {, and } 24 \mathrm{~h}\end{array}$ & $\begin{array}{l}\text { - Pelargonidin-3-glucoside } \\
\text { - Pelargonidin glucuronide } \\
\text { (3 isomers) }\end{array}$ & [22] \\
\hline
\end{tabular}


Table 1 List of studies reporting candidate biomarkers for grape/raisin and berry consumption (Continued)

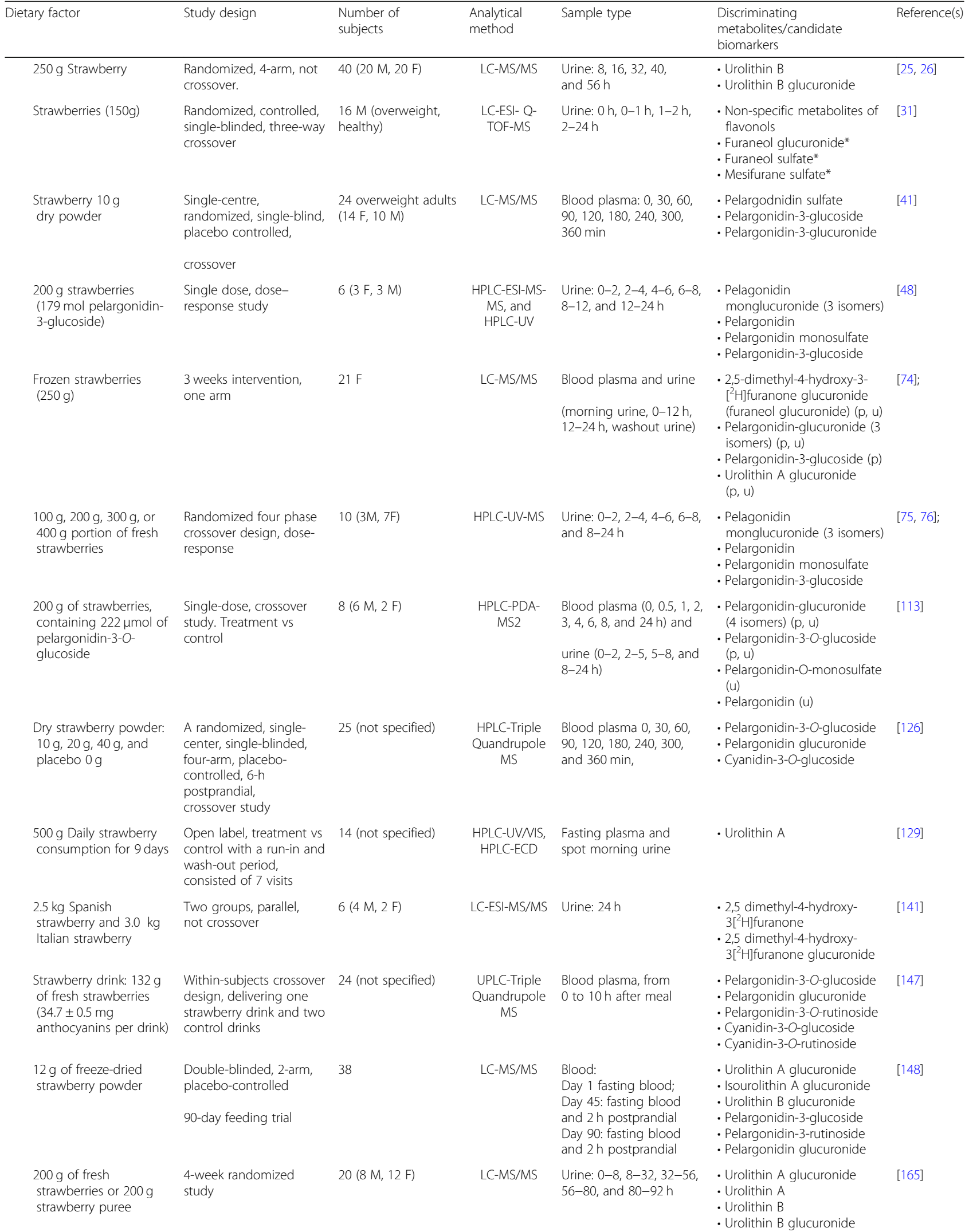


Table 1 List of studies reporting candidate biomarkers for grape/raisin and berry consumption (Continued)

\begin{tabular}{|c|c|c|c|c|c|c|}
\hline Dietary factor & Study design & $\begin{array}{l}\text { Number of } \\
\text { subjects }\end{array}$ & $\begin{array}{l}\text { Analytical } \\
\text { method }\end{array}$ & Sample type & $\begin{array}{l}\text { Discriminating } \\
\text { metabolites/candidate } \\
\text { biomarkers }\end{array}$ & Reference(s) \\
\hline $\begin{array}{l}\text { Strawberry milk } \\
\text { beverage and } \\
\text { strawberry water } \\
\text { beverage; } 12 \mathrm{~g} \text { of } \\
\text { powder in each drink }\end{array}$ & $\begin{array}{l}\text { Single-center, } \\
\text { randomized, single- } \\
\text { blinded, crossover study }\end{array}$ & 9 & & $\begin{array}{l}\text { Blood } 0 \mathrm{~h}, 0.25 \mathrm{~h}, 0.5 \mathrm{~h}, \\
1 \mathrm{~h}, 1.5 \mathrm{~h}, 2 \mathrm{~h}, 3 \mathrm{~h}, 4 \mathrm{~h}, \\
5 \mathrm{~h}, 6 \mathrm{~h}\end{array}$ & $\begin{array}{l}\text { - Pelargonidin-3-glucoside } \\
\text { - Pelargonidin-3-rutinoside } \\
\text { - Pelargonidin glucuronide }\end{array}$ & [179] \\
\hline \multicolumn{7}{|l|}{ Raspberry } \\
\hline $300 \mathrm{~g}$ of raspberries & Acute, 1-arm study & $\begin{array}{l}10 \text { healthy } \\
\text { persons } \\
(6 \mathrm{M}, 4 \mathrm{~F}) \text {; } \\
4 \text { with } \\
\text { ileostomy }\end{array}$ & $\begin{array}{l}\text { HPLC-PDA- } \\
\quad \text { MS2 }\end{array}$ & $\begin{array}{l}\text { Blood plasma: 0, 1.0, 1.5, } \\
2.0,3.0,5.0,6.5,7.5 \text {, and } \\
24 \mathrm{~h} \\
\text { Urine: } 0-4 \mathrm{~h}, 4-7 \mathrm{~h} \text {, } \\
7-24 \mathrm{~h}, 24-32 \mathrm{~h} \text {, and } \\
32-48 \mathrm{~h} \\
\text { Ileal fluid: } 0-4 \mathrm{~h}, 4-7 \mathrm{~h} \text {, } \\
7-24 \mathrm{~h} \text {, and } 24-48 \mathrm{~h}\end{array}$ & $\begin{array}{l}\text { - Cyanidin-3-O-(2"-O-gluco } \\
\text { syl)rutinoside (if, u) } \\
\text { - Cyanidin-3-O-(2"-O-xylosyl) } \\
\text { rutinoside (if) } \\
\text { - Cyanidin-3-O-glucoside } \\
\text { (if, u) } \\
\text { - Cyanidin-3-O-rutinoside (if) } \\
\text { - Cyanidin-3-O-sophoroside } \\
\text { (if, u) } \\
\text { - Ellagic acid(if, u) } \\
\text { - Ellagic acid-O-glucuronide } \\
\text { (u) } \\
\text { - Pelargonidin-3-O-(2"-O-glu } \\
\text { cosyl)rutinoside (if) } \\
\text { - Pelargonidin-3-O-glucoside } \\
\text { (if) } \\
\text { - Pelargonidin-3-O-sophoro } \\
\text { side (if) } \\
\text { - Sanguiin H-6 (if) } \\
\text { - Urolithin A glucuronide (u) }\end{array}$ & [70] \\
\hline $\begin{array}{l}200 \mathrm{~g}, 400 \mathrm{~g} \text { of } \\
\text { raspberries, + placebo }\end{array}$ & $\begin{array}{l}\text { 3-arm double-blind } \\
\text { randomized controlled } \\
\text { crossover }\end{array}$ & $10 \mathrm{M}$ & UPLC-QTOF & $\begin{array}{l}\text { Blood: } 0 \mathrm{~h}, 2 \mathrm{~h} \text { and } 24 \mathrm{~h} \\
\text { postprandial }\end{array}$ & $\begin{array}{l}\text { - Urolithin A } \\
\text { - Urolithin A-3-glucuronide } \\
\text { - Urolithin A-sulfate } \\
\text { - Urolithin B glucuronide } \\
\text { - (Iso)urolithin A } \\
\text { - (Iso)urolithin A-3- } \\
\text { glucuronide }\end{array}$ & [78] \\
\hline $\begin{array}{l}300 \mathrm{~g} \text { of homogenized } \\
\text { raspberries }\end{array}$ & Acute, single dose & $9(4 \mathrm{M}, 5 \mathrm{~F})$ & $\begin{array}{l}\text { UHPLC-MSn } \\
\text { and UHPLC- } \\
\text { MS2 }\end{array}$ & $\begin{array}{l}\text { Urine: } 0-4 \mathrm{~h}, 4-8 \mathrm{~h}, \\
8-24 \mathrm{~h}, 24-32 \mathrm{~h}, 32-48 \mathrm{~h} \\
\text { Blood plasma: } 0.5 \mathrm{~h}, 1 \mathrm{~h}, \\
1.5 \mathrm{~h}, 2 \mathrm{~h}, 3 \mathrm{~h}, 4 \mathrm{~h}, 5 \mathrm{~h}, 6 \\
\mathrm{~h}, 8 \mathrm{~h}, 24 \mathrm{~h}\end{array}$ & $\begin{array}{l}\text { - (Iso)urolithin A-sulfate-O- } \\
\text { glucuronide (u, p) } \\
\text { - Isourolithin A-O-glucuronide } \\
\text { (u, p) } \\
\text { - Peonidin-3-O-glucoside (u) } \\
\text { - Urolithin A (u) } \\
\text { - Urolithin A-O-glucuronide } \\
\text { (u, p) } \\
\text { - Urolithin A-sulfate (u, p) } \\
\text { - Urolithin B (u) } \\
\text { - Urolithin B-3-O-glucuronide } \\
\text { (u, p) } \\
\text { - Dimethylellagic acid-O- } \\
\text { glucuronide (u, p) } \\
\text { - Cyanidin-3-O-glucoside } \\
\text { (u, p) } \\
\text { - Cyanidin-3-O-glucuronide } \\
\text { (p) } \\
\text { - Peonidin-3-O-glucoside (u) }\end{array}$ & [98] \\
\hline $\begin{array}{l}\text { Intragastric dosage of } \\
\text { raspberry ketone, } 1 \\
\mathrm{mmol} / \mathrm{kg}\end{array}$ & Single dose & (not specified) & $\begin{array}{l}\text { GC-MS after } \\
\text { derivatization }\end{array}$ & $\begin{array}{l}\text { Urine of rat,rabbit, } \\
\text { and guinea pig }\end{array}$ & $\begin{array}{l}\cdot \text { 2-(4-hydroxyphenyl)ethanol } \\
\cdot \text { 4-(4-hydroxyphenpl)butan- } \\
\text { 2-one } \\
\cdot \text { 4-hydroxybenzoic acid } \\
\cdot \text { 4-hydroxyphenylacetic acid } \\
\cdot \text { 4-(4-hydroxyphenyl)butan- } \\
\text { 2-ol } \\
\cdot \text { 4-(4-hydroxy-3- } \\
\text { methoxyphenyl)butan-2- } \\
\text { one } \\
\cdot \text { 4-hydroxy-4-(4- } \\
\text { hydroxyphenyl)butan-2-one } \\
\cdot 3-(4- \\
\text { hydroxyphenyl)propionic } \\
\text { acid } \\
\cdot \text { 4-(3,4- } \\
\text { dihydroxyphenyl)butan-2- } \\
\text { one } \\
\cdot \text { 4-(4-hydroxy-3- } \\
\text { methoxpphenyl)butan-2-ol }\end{array}$ & [156] \\
\hline
\end{tabular}


Table 1 List of studies reporting candidate biomarkers for grape/raisin and berry consumption (Continued)

\begin{tabular}{|c|c|c|c|c|c|c|}
\hline Dietary factor & Study design & $\begin{array}{l}\text { Number of } \\
\text { subjects }\end{array}$ & $\begin{array}{l}\text { Analytical } \\
\text { method }\end{array}$ & Sample type & $\begin{array}{l}\text { Discriminating } \\
\text { metabolites/candidate } \\
\text { biomarkers }\end{array}$ & Reference(s) \\
\hline & & & & & $\begin{array}{l}\cdot \text { - 4-(4-hydroxyphenyl)butan-2, } \\
\text { 3-diol } \\
\cdot \text { 4-(3,4- } \\
\text { dihydroxyphenyl)butan-2-ol } \\
\cdot \text { 1-hydroxy-4-(4-hydroxphe } \\
\text { nyl)butan-2-one } \\
\cdot \text { 4-(4-hydroxyphenyl)butan-l, } \\
\text { 2-diol }\end{array}$ & \\
\hline $\begin{array}{l}125 \mathrm{~g} \text { raspberries per } \\
\text { day for } 4 \text { weeks. }\end{array}$ & Not specified & 3 & $\begin{array}{l}\text { UHPLC-QTOF } \\
\text { (MSSMS); } \\
\text { UHPLC-QQQ } \\
\text { (MS/MS) }\end{array}$ & $\begin{array}{l}\text { Blood: } 0 \mathrm{~h}, 0.5 \mathrm{~h}, 1 \mathrm{~h}, \\
2 \mathrm{~h}, 3 \mathrm{~h}, 4 \mathrm{~h}, 5 \mathrm{~h}, 5.5 \mathrm{~h}, \\
6 \mathrm{~h}, 7 \mathrm{~h}, 8 \mathrm{~h} \text {, and } 24 \mathrm{~h} \\
\text { Urine: }-12 \mathrm{~h}-0 \mathrm{~h} ; 0-4 \mathrm{~h}, \\
4-8 \mathrm{~h}, 8-24 \mathrm{~h}, 24-36 \mathrm{~h}, \\
36-48 \mathrm{~h}\end{array}$ & $\begin{array}{l}\text { - Cyanidin-3-O-sophoroside } \\
\text { - Cyanidin-3-O-(2G } \\
\text { glucosylrutinoside) } \\
\text { - Cyanidin-3-O-glucoside } \\
\text { - Pelargonidin-3-O- } \\
\text { sophoroside } \\
\text { - Cyanidin-3-O-rutinoside } \\
\text { - Cyanidin-3-O-glucuronide } \\
\text { - Methyl cyanidin-3-O- } \\
\text { sophoroside } \\
\text { - Peonidin-3-O-glucoside } \\
\text { - Urolithin A glucuronide } \\
\text { - Urolithin B glucuronide } \\
\text { - Urolithin A }\end{array}$ & [184] \\
\hline \multicolumn{7}{|l|}{ Blackberry } \\
\hline $\begin{array}{l}200 \mathrm{~g} \text { of blackberries } \\
\text { (containing } 960 \mu \mathrm{mol} \text { of } \\
\text { anthocyanins expressed } \\
\text { as cyanidin 3-glucoside } \\
\text { equivalents) }\end{array}$ & $\begin{array}{l}\text { Acute, single dose, } \\
\text { kinetic study }\end{array}$ & $5(3 F, 2 M)$ & $\begin{array}{l}\text { HPLC-ESI-MS- } \\
\text { MS and HPLC } \\
\text { with UV-vis }\end{array}$ & $\begin{array}{l}\text { Urine: } 0-2,2-4,4-6,6-8 \text {, } \\
8-12 \text {, and } 12-24 \mathrm{~h}\end{array}$ & $\begin{array}{l}\text { - Cyanidin glycosides } \\
\text { - Cyanidin glucuronide } \\
\text { - Cyanidin diglucuronide } \\
\text { - Cyanidin sulfate } \\
\text { - Peonidin glycosides } \\
\text { - Peonidin glucuronide, } \\
\text { - Peonidin-3-glucoside } \\
\text { glucuronide }\end{array}$ & [47] \\
\hline $\begin{array}{l}250 \mathrm{~mL} \text { of tropical } \\
\text { highland blackberry } \\
\text { juice }\end{array}$ & $\begin{array}{l}\text { Acute study, repeated } \\
\text { over the year, no } \\
\text { placebo, different } \\
\text { kinetics design }\end{array}$ & $\begin{array}{l}26(16 \mathrm{~F} \\
10 \mathrm{M})\end{array}$ & $\begin{array}{l}\text { UPLC-DAD/ } \\
\text { ESI-Q-TOF/ } \\
\text { MS2 }\end{array}$ & Spot urine samples & $\begin{array}{l}\text { - Urolithin A } \\
\text { - Urolithin B } \\
\text { - Urolithin C } \\
\text { - Urolithin D } \\
\text { - Urolithin M5 } \\
\text { - Methyl urolithin A } \\
\text { - Urolithin glucuronide A } \\
\text { - Urolithin glucuronide B } \\
\text { - Urolithin glucuronide C } \\
\text { - Urolithin glucuronide D } \\
\text { - Ellagic acid } \\
\text { - Ellagic acid dimethyl ether } \\
\text { glucuronide } \\
\text { - Ellagic acid dimethyl ether }\end{array}$ & {$[64,65]$} \\
\hline $\begin{array}{l}\text { Freeze-dried } \\
\text { blackberries: } 32 \mathrm{~g} \text { for } \\
\text { females and } 45 \mathrm{~g} \text { for } \\
\text { males }\end{array}$ & $\begin{array}{l}26 \text { weeks pilot study } \\
\text { with daily consumption } \\
\text { of supplement }\end{array}$ & 20 & LC-MS/MS & $\begin{array}{l}3 \mathrm{~h} \text { pooled urine } \\
\text { collected in the } \\
\text { morning: week } 0 \text {, } \\
\text { week } 12 \text {, week } 26\end{array}$ & $\begin{array}{l}\text { - Urolithin A glucuronide } \\
\text { - Urolithin A-sulfate } \\
\text { - Dimethylellagic acid } \\
\text { glucuronide }\end{array}$ & [89] \\
\hline $250 \mathrm{~g}$ of blackberries & $\begin{array}{l}\text { Randomized and } \\
\text { crossover intervention }\end{array}$ & $\begin{array}{l}18 \text { (not } \\
\text { specified) }\end{array}$ & HPLC-DAD & $\begin{array}{l}\text { Blood plasma: } 15,30, \\
60 \text {, and } 12 \mathrm{~min} \\
\text { Urine: } 0,120 \mathrm{~min}\end{array}$ & $\begin{array}{l}\text { - Cyanidin glycosides (u) } \\
\text { - Cyanidin glucoside } \\
\text { glucuronide ( }(u, p) \\
\text { - Cyanidin glucoside sulfate } \\
\text { ( } p, u) \\
\text { - Cyanidin glucuronide (two } \\
\text { isomers) }(p, u) \\
\text { - Methyl cyanidin glucoside } \\
\text { glucuronide }(p, u) \\
\text { - Methyl cyanidin } \\
\text { glucuronide }(p, u)\end{array}$ & [100] \\
\hline $\begin{array}{l}20 \mathrm{~g} \text { freeze-dried } \\
\text { blackberry powder, } \\
\text { three times a day, for } \\
9 \text { weeks }\end{array}$ & $\begin{array}{l}\text { One-arm study, patients } \\
\text { with colorectal cancer }\end{array}$ & $\begin{array}{l}20(17 \mathrm{M} \\
3 \mathrm{~F})\end{array}$ & $\begin{array}{l}\text { UHPLC-MS/ } \\
\text { MS, Orbitrap } \\
\text { LTQ, and GC- } \\
\text { MS }\end{array}$ & $\begin{array}{l}\text { Blood plasma, urine at } \\
\text { baseline and after } \\
\text { treatment }\end{array}$ & - Cyanidin glycosides (u) & [123] \\
\hline $\begin{array}{l}45 \mathrm{~g} \text { of freeze-dried } \\
\text { blackberries }\end{array}$ & $\begin{array}{l}\text { One arm, } 1 \text { week study } \\
\text { with } 45 \mathrm{~g} \text { of meal daily }\end{array}$ & $10 \mathrm{M}$ & $\begin{array}{l}\text { LC-MS/UV-Vis; } \\
\text { HPLC-ESI-MS/ } \\
\text { MS }\end{array}$ & $\begin{array}{l}\text { Urine: } 4 \mathrm{~h}, 8 \mathrm{~h}, 12 \mathrm{~h} \text {, at } \\
1 \mathrm{st} \text { and } 7 \text { th day }\end{array}$ & - Cyanidin glycosides & [161] \\
\hline
\end{tabular}


Table 1 List of studies reporting candidate biomarkers for grape/raisin and berry consumption (Continued)

\begin{tabular}{|c|c|c|c|c|c|c|}
\hline Dietary factor & Study design & $\begin{array}{l}\text { Number of } \\
\text { subjects }\end{array}$ & $\begin{array}{l}\text { Analytical } \\
\text { method }\end{array}$ & Sample type & $\begin{array}{l}\text { Discriminating } \\
\text { metabolites/candidate } \\
\text { biomarkers }\end{array}$ & Reference(s) \\
\hline \multicolumn{7}{|l|}{ Cranberry } \\
\hline Juice $480 \mathrm{~mL}$ cranberry & $\begin{array}{l}\text { Acute, single-dose, } \\
\text { kinetic study }\end{array}$ & $15(13 \mathrm{M}, 2 \mathrm{~F})$ & $\begin{array}{l}\text { LC-Diode } \\
\text { array/ ion trap } \\
\text { MS/MS }\end{array}$ & $\begin{array}{l}\text { Blood plasma: } 0 \mathrm{~h}, 1 \mathrm{~h}, \\
2 \mathrm{~h}, \mathrm{~h}, 4 \mathrm{~h} \\
\text { urine: } 0-4 \mathrm{~h}\end{array}$ & $\begin{array}{l}\text { - Cyanidin-3-glycosides }(u, p) \\
\text { - Malvidine-3-glycosides }(u, p) \\
\text { - Peonidin-3-glycosides }(u, p)\end{array}$ & {$[108] ;$} \\
\hline $200 \mathrm{~mL}$ cranberry juice & $\begin{array}{l}\text { Acute, single-dose, } \\
\text { kinetic study }\end{array}$ & $11(9 \mathrm{M}, 2 \mathrm{~F})$ & $\begin{array}{l}\text { HPLC-ESI-MS- } \\
\text { MS }\end{array}$ & Urine $24 \mathrm{~h}$ & $\begin{array}{l}\text { - Cyanidin-3-glycosides } \\
\text { - Malvidine-3-glycosides } \\
\text { - Peonidin-3-glycosides }\end{array}$ & {$[119]$} \\
\hline $\begin{array}{l}250 \mathrm{~mL} \text { cranberry juice } \\
\text { twice a day }\end{array}$ & Crossover, 2-arm study & $17 \mathrm{~F}$ & $\begin{array}{l}\text { UHPLC-Q- } \\
\text { Orbitrap- } \\
\text { HRMS }\end{array}$ & $\begin{array}{l}\text { Blood: fasting base line, } \\
2 \mathrm{~h} \text { postprandial on third } \\
\text { consumption day }\end{array}$ & $\begin{array}{l}\text {-5-(trihydroxphenyl)- } \gamma^{-} \\
\text {valerolactone* } \\
\text { - Phenolics derivatives* }\end{array}$ & [94] \\
\hline $450 \mathrm{~mL}$ & $\begin{array}{l}\text { Double-blind, placebo- } \\
\text { controlled parallel trial }\end{array}$ & 78 & LC-MS/MS & $\begin{array}{l}\text { Urine: } 0 \mathrm{~h}, 3 \mathrm{~h}, 5 \mathrm{~h}, 8 \mathrm{~h} \\
\text { postprandial }\end{array}$ & $\begin{array}{l}\text { - Cyanidin-3-glucoside } \\
\text { - Peonidin-3-glucoside } \\
\text { - Cyanidin-3-galactoside } \\
\text { - Peonidin-3-galactoside } \\
\text { - Peonidin-3-arabinoside } \\
\text { - Cyanidin-3-arabinoside }\end{array}$ & [27] \\
\hline $\begin{array}{l}2 \text { types of capsules of } \\
\text { cranberry extract, intake } \\
\text { for } 7 \text { days; } 36 \mathrm{mg} \text { vs } 12 \\
\text { mg extract per capsule }\end{array}$ & $\begin{array}{l}\text { Randomized, double- } \\
\text { blind, 2-arm repeated } \\
\text { measure crossover } \\
\text { design }\end{array}$ & 13 & $\begin{array}{l}\text { LC-LTQ- } \\
\text { Orbitrap XL }\end{array}$ & $\begin{array}{l}\text { Urine: } 0 \mathrm{~h}, 1 \mathrm{~h}, 2 \mathrm{~h}, 4 \mathrm{~h} \text {, } \\
6 \mathrm{~h}, 10 \mathrm{~h}, 12 \mathrm{~h}, 24 \mathrm{~h} \text {, at } \\
\text { day } 0 \text { and day } 7 \mathrm{th}\end{array}$ & $\begin{array}{l}\text { - Hydroxyphenyl- } \\
\text { valerolactone derivatives* }\end{array}$ & [7] \\
\hline \multicolumn{7}{|l|}{ Blackcurrant } \\
\hline $\begin{array}{l}100 \mathrm{~g} \text { whole } \\
\text { blackcurrant vs } 50 \mathrm{~g} \\
\text { diluted blackcurrant } \\
\text { syrup }\end{array}$ & $\begin{array}{l}\text { Randomized two-phase } \\
\text { crossover }\end{array}$ & $10(3 \mathrm{M}, 7 \mathrm{~F})$ & HPLC-ESI-MS & $\begin{array}{l}\text { Blood plasma: } 15 \mathrm{~min}, 30 \\
\mathrm{~min}, 45 \mathrm{~min} \text { and } 1 \mathrm{~h}, 1.5 \\
\mathrm{~h}, 2 \mathrm{~h}, 3 \mathrm{~h}, 4 \mathrm{~h}, 6 \mathrm{~h}, 8 \mathrm{~h} \text {, } \\
24 \mathrm{~h} \text {, and } 48 \mathrm{~h} \\
\text { Urine: } 0-2 \mathrm{~h}, 2-4 \mathrm{~h}, 4-6 \mathrm{~h} \text {, } \\
6-8 \mathrm{~h}, 8-24 \mathrm{~h} \text { and } \\
24-48 \mathrm{~h}\end{array}$ & $\begin{array}{l}\text { - Cyanidin-3-O-rutinoside (u) } \\
\text { - Delphinidin 3-O-rutinoside } \\
\text { (u) }\end{array}$ & {$[75,76]$} \\
\hline $\begin{array}{l}250 \mathrm{~mL} \text { black currant } \\
\text { juice vs placebo drink }\end{array}$ & $\begin{array}{l}\text { Randomized, cross-over, } \\
\text { double-blind, placebo- } \\
\text { controlled acute meal } \\
\text { study }\end{array}$ & $20(11 F, 9 M)$ & GC-MS & $\begin{array}{l}\text { urine: collected every } \\
2 \mathrm{~h} \text { and at } 24 \mathrm{~h}\end{array}$ & $\begin{array}{l}\text { - Cyanidin-3-O-rutinoside } \\
\text { - Delphinidin 3-O-glucoside } \\
\text { - Delphinidin 3-O-rutinoside }\end{array}$ & {$[80]$} \\
\hline $\begin{array}{l}300 \mathrm{~mL} \text { blackcurrant } \\
\text { extract }\end{array}$ & Acute crossover & $5 \mathrm{M}$ & HPLC-UV & $\begin{array}{l}\text { Urine: } 0 \mathrm{~h}, 60 \mathrm{~min}, 120 \\
\mathrm{~min}, 180 \mathrm{~min}, 240 \mathrm{~min}, \\
300 \mathrm{~min}\end{array}$ & $\begin{array}{l}\text { - Cyanidin-3-O-rutinoside } \\
\text { - Cyanidin-3-O-glucoside } \\
\text { - Delphinidin 3-O-glucoside } \\
\text { - Delphinidin 3-O-rutinoside }\end{array}$ & {$[105]$} \\
\hline $\begin{array}{l}253 \mathrm{mg} \text { of blackcurrant } \\
\text { extracts }\end{array}$ & Acute single dose & $4(3 F, 1 M)$ & LC-MS/MS & $\begin{array}{l}\text { Blood plasma: } 0 \mathrm{~h}, 0.5 \mathrm{~h}, \\
1 \mathrm{~h}, 2 \mathrm{~h}, 3 \mathrm{~h}, 4 \mathrm{~h}, 6 \mathrm{~h}\end{array}$ & $\begin{array}{l}\text { - Cyanidin-3-O-rutinoside } \\
\text { - Cyanidin-3-O-rutinoside } \\
\text { - Delphinidin 3-O-glucoside } \\
\text { - Delphinidin 3-O-rutinoside }\end{array}$ & [114] \\
\hline $\begin{array}{l}200 \mathrm{~mL} \text { blackcurrant } \\
\text { juice }\end{array}$ & $\begin{array}{l}\text { Acute, single-dose, } \\
\text { kinetic study }\end{array}$ & $4(2 \mathrm{M}, 2 \mathrm{~F})$ & HPLC-UV-VIS & $\begin{array}{l}\text { Urine: } 0 \mathrm{~h}, 0.5 \mathrm{~h}, 1 \mathrm{~h}, \\
1.5 \mathrm{~h}, 2 \mathrm{~h}, 2.5 \mathrm{~h}, 3 \mathrm{~h}, \\
3.5 \mathrm{~h}, 4 \mathrm{~h}, 4.5 \mathrm{~h}, 5 \mathrm{~h}\end{array}$ & $\begin{array}{l}\text { - Cyanidin-3-O-rutinoside } \\
\text { - Cyanidin-3-O-glucoside } \\
\text { - Delphinidin 3-O-glucoside } \\
\text { - Delphinidin 3-O-rutinoside }\end{array}$ & {$[115]$} \\
\hline $\begin{array}{l}1.6 \mathrm{~g} \text { blackcurrant } \\
\text { extract powder in } \\
200 \mathrm{~mL} \text { water }\end{array}$ & $\begin{array}{l}\text { Acute single-dose } \\
\text { kinetic study }\end{array}$ & $5 \mathrm{M}$ & $\begin{array}{l}\text { HPLC-ESI- } \\
\text { MS/MS }\end{array}$ & $\begin{array}{l}\text { Plasma } 0 \mathrm{~h}, 1 \mathrm{~h}, 2 \mathrm{~h}, 4 \mathrm{~h} \text {, } \\
6 \mathrm{~h}, 8 \mathrm{~h} \\
\text { Urine: } 24-0 \mathrm{~h} \text { prior to } \\
\text { ingestion; } 0-2 \mathrm{~h}, 2-4 \mathrm{~h} \text {, } \\
4-6 \mathrm{~h}, 6-8 \mathrm{~h}, 8-12 \mathrm{~h}, \\
12-24 \mathrm{~h}, \\
24-48 \mathrm{~h} \text { postprandial }\end{array}$ & $\begin{array}{l}\text { - Delphinidin 3-O-rutinoside } \\
\text { - Delphinidin 3-O- } \\
\text { glucuronide } \\
\text { - Cyanidin-3-O-glucoside } \\
\text { - Cyanidin 3-O-glucuronide }\end{array}$ & [139] \\
\hline
\end{tabular}

aroma compounds. Supplementary Table 1 reports the composition of various berries, highlighting the similarities among them, as well as some characteristic compounds, especially specific aroma compounds, which could potentially be of interest as candidate BFIs.

In the case of anthocyanins, the parent glycosides as well as the corresponding aglycones cyanidin, delphinidin, pelargonidin, malvidin, and peonidin have been analyzed, while their sulfates and glucuronide conjugates were rarely quantified because of the lack of commercial standards. Only four studies included sulfate and glucuronide conjugates of cyanidin, peonidin, delphinidin, and petunidin in the analysis [5, 47, 81, 98]

Ellagitannins were the second family of interest found in the literature related to non-botanical berry consumption. The most frequently reported ellagitannin metabolites included ellagic acid and several microbial metabolites, such as urolithin A, urolithin B, urolithin $\mathrm{C}$, 
Table 2 List of non-retained compounds for grapes/raisins and berries

\begin{tabular}{ll}
\hline Food item & Compound \\
\hline Raisin & p-Hydroxybenzoic acid \\
Grape & Anthocyanins and its derivatives: \\
& cyanidin-3-glucoside, cyanidin-3- \\
& glucuronide, \\
& delphinidin-3-glucoside, delphinidin- \\
& 3-glucuronide, \\
& malvidin-3-glucoside, malvidin-3- \\
& glucuronide, \\
& peonidin-3-glucoside, peonidin-3- \\
& glucuronide, \\
& petunidin-3-glucoside, petunidin-3- \\
& glucuronide
\end{tabular}

\section{Flavan-3-ol metabolites and its derivatives:}

catechin, catechin glucuronide, catechin sulfate, catechin sulfate glucuronide, epicatechin, epicatechin glucuronide, epicatechin sulfate, epicatechin sulfate glucuronide, epicatechin-3-O-gallate, O-methyl(epi)catechin-O-glucuronide,

O-methyl-(epi)catechin-O-sulfate, gallocatechin, epigallocatechin, epigallocatechin-O-glucuronide, O-methyl-(epi)gallocatechin-O-sulfate, 4"-O-methyl-(epi)gallocatechin-Osulfate, 4-hydroxy-5-(3,4-

dihydroxyphenyl)valeric acid, 4hydroxy-5-(dihydroxyphenyl)-valeric acid glucuronide, 4-hydroxy-5-(dihy droxyphenyl)-valeric acid sulfate, 5-(3,4'-Dihydroxyphenyl)-g-

valerolactone, 5-(3',4'-

dihydroxyphenyl)- $\gamma$-valerolactone, 5-

(3',5'-dihydroxyphenyl)-- $\gamma$-valerolactone,

5-(dihydroxyphenyl)- $\gamma-$

valerolactone glucuronide, 5-

(hydroxyphenyl)- $-\gamma$-valerolactone glucuronide, 5-(hydroxy-methoxyphenyl)- $\gamma$-valerolactone glucuronide

Procyanidin B1

Benzoic acids and its derivatives: gallic acid, O-methylgallic acid, $\mathrm{O}$ methylgallic acid-3-O-sulfate, 3,4-Odimethylgallic acid, hydroxybenzoic acid, hydroxy-dimethoxybenzoic acid glucuronide, 3,4-dihydroxybenzoic acid, 3-methoxy-4-hydroxybenzoic aicd, 3,5-dimethoxy-4-

hydroxybenzoic aicd, syringic acid, vanillic acid, vanillic acid-glucuronide, vanillic acid 4-sulfate, isovanillic acid, homovanillic acid, homovanillic acid 4-sulfate, pyrogallol, 2-O-

methylpyrogallol,

protocatechuic-O-glucoside,

hippuric acid, hydroxyhippuric acid

Quercetin and its derivatives: quercetin, quercetin glucuronide, 3'-O-methyl quercetin,

dihydroquercetin

Cinnamic acids and its derivtives: caffeic acid, caffeic acid sulfate,

\begin{tabular}{lll} 
Biofluid & Reasons for exclusion & References \\
\hline Plasma & Unspecific & {$[84]$} \\
$\begin{array}{l}\text { Plasma/ urine/ } \\
\text { ileal fluid }\end{array}$ & $\begin{array}{l}\text { Unspecific, low } \\
\text { bioavailability }\end{array}$ & {$[19,55,15,157]$}
\end{tabular}

Plasma/ urine/ Unspecific, common for ileal fluid many fruits and vegetables

$[171,157,158,99,85,150,117,23,69,29]$

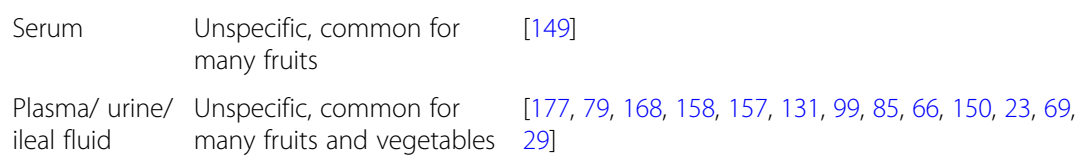

Plasma/ urine Unspecific, common for many fruits and vegetables
Plasma/ urine/ Unspecific, common for ileal fluid
$[79,168,157,150,23,69,29]$ 
Table 2 List of non-retained compounds for grapes/raisins and berries (Continued)

\begin{tabular}{|c|c|c|c|c|}
\hline Food item & Compound & Biofluid & Reasons for exclusion & References \\
\hline & $\begin{array}{l}\text { dihydrocaffeic acid, dihydrocaffeic } \\
\text { acid glucuronide, dihydrocaffeic acid } \\
\text { sulfate, } \\
\text { ferulic acid, ferulic acid sulfate, } \\
\text { dihydroferulic acid, isoferulic acid } \\
\text { glucuronide, isoferulic acid sulfate, } \\
\text { dihydroferulic acid, dihydroferulic } \\
\text { acid glucuronide, dihydroferulic acid } \\
\text { sulfate } \\
\text { p-coumaric acid, p-coumaric acid } \\
\text { sulfate, m-dihydrocoumaric acid, } \\
\text { dihydrocoumaric acid-O-sulfate, } \\
\text { trans-caftaric acid, trans-coutaric } \\
\text { acid, trans-fertaric acid, } \\
\text { dihydrosinapic acid glucuronide }\end{array}$ & & many fruits and vegetables & \\
\hline & $\begin{array}{l}\text { Phenylacetic-, and } \\
\text { phenylpropionic acids and its } \\
\text { derivatives: } \\
\text { 2-hydroxyphenylacetic acid, } \\
\text { 3-hydroxyphenylacetic acid, } \\
\text { 4-hydroxyphenylacetic acid, } \\
\text { 3,4-dihydroxyphenylacetic acid, } \\
\text { 3,4-dihydroxyphenylacetic acid, } \\
\text { hydroxyphenylacetic acid, } \\
\text { hydroxyphenylpropionic acid, } \\
\text { dihydroxyphenylpropionic acid, } \\
\text { dihydrosinapic acid glucuronide, } \\
\text { hydroxyphenylpropionic aicd, } \\
\text { dihydroxyphenylpropionic acid }\end{array}$ & Plasma/urine & $\begin{array}{l}\text { Unspecific, common for } \\
\text { many fruits and vegetables }\end{array}$ & {$[177,168,158,85,150,69]$} \\
\hline & $\begin{array}{l}\text { Mandelic acid metabolites } \\
\text { mandelic acid, 4-hydroxymandelic } \\
\text { acid, vanilmandelic acid }\end{array}$ & Urine & Unspecific & $[168,158,69])$ \\
\hline & $\begin{array}{l}\text { Other metabolites: } \\
\text { feruloylglycine, vanilloylglycine, } \\
\text { 6-sulfatoxymelatonin, enterodiol, } \\
\text { tyrosine }\end{array}$ & Urine & Unspecific & {$[79,71,157,85]$} \\
\hline & Total phenols, conjugated phenols & Plasma & Unspecific & {$[118,6]$} \\
\hline & Naringenin & Urine & Unspecific & [79] \\
\hline & Urolithin A & Plasma & $\begin{array}{l}\text { Unspecific, common for } \\
\text { many fruits and nuts }\end{array}$ & [122] \\
\hline Blueberry & $\begin{array}{l}\text { Anthocyanidins, and its } \\
\text { derivatves: } \\
\text { cyanidin, cyanidin glucuronide, } \\
\text { cyanidin glycoside, cyanidin methyl } \\
\text { glucoside, cyanidin methyl } \\
\text { glucuronide, } \\
\text { delphinidin, delphinidin glucuronide, } \\
\text { delphinidin glycoside, delphinidin } \\
\text { glycoside methyl glucuronide, } \\
\text { delphinidin methyl glucuronide, } \\
\text { delphinidin methyl glycoside, } \\
\text { malvidin 3-glucoside, malvidin } \\
\text { glucuronide, malvidin glycoside, } \\
\text { malvidin glycoside glucuronide, } \\
\text { malvidin glycoside sulfate, malvidin } \\
\text { methyl glucuronide, malvidin methyl } \\
\text { glycoside, pelargonidin glycoside, } \\
\text { pelargonidin glucuronide, } \\
\text { pelargonidin glycoside sulfate, } \\
\text { pelargonidin methyl glucuronide, } \\
\text { pelargonidin methyl glycoside, } \\
\text { pelargonidin, } \\
\text { peonidin, peonidin glucuronide, } \\
\text { peonidin glycoside, peonidin }\end{array}$ & Urine, plasma & $\begin{array}{l}\text { Unspecific, common for } \\
\text { many fruits and vegetables, } \\
\text { low bioavailability }\end{array}$ & {$[37,80-83,103,105,178,112]$} \\
\hline
\end{tabular}


Table 2 List of non-retained compounds for grapes/raisins and berries (Continued)

\begin{tabular}{ll}
\hline Food item & Compound \\
\hline & glycoside sulfate, peonidin methyl \\
& glucuronide, petunidin, petunidin \\
glucuronide, petunidin glycoside, & petunidin glycoside glucuronide, \\
petunidin glycoside methyl, \\
petunidin glycoside methyl \\
glucuronide, petunidin methyl \\
glucuronide \\
Flavan-3-ol metabolites and \\
its derivatives: \\
(4r)-5-(3-hydroxyphenyl)- \\
valerolactone-4-O-sulfate, \\
hydroxyphenyl-valerolactone*, \\
dihydroxyphenyl-valerolactone, \\
hydroxy-(dihydroxyphenyl) pentenoic \\
acid glucuronide*, hydroxy- \\
(dihydroxyphenyl) valeric acid \\
glucuronide I *, hydroxy- \\
(dihydroxyphenyl) valeric acid \\
glucuronide II *, hydroxy- \\
(dihydroxyphenyl) valeric acid \\
sulphate*, hydroxy-(dihydroxyphenyl) \\
valeric acid glucuronide I ${ }^{*}$, \\
hydroxy-(dihydroxyphenyl) valeric \\
acid glucuronide II*, hydroxy- \\
(dihydroxyphenyl) valeric acid \\
sulphate*
\end{tabular}

Benzoic acids and its derivatives: benzoic acid, 2,3-dihydroxybenzoic acid, 2,4-dihydroxybenzoic acid, 2,5-dihydroxybenzoic acid, 2-

hydroxybenzoic acid, 3hydroxybenzoic acid, 4-

hydroxybenzoic acid, 3methoxybenzoic acid-3-sulfates

4-methoxybenzoic acid-4-sulfates

3,4-dihydroxybenzaldehyde,

3,5-dihydroxybenzyl alcohol, 3,5-

dimethoxybenzoic acid methyl ester,

4-methylcatechol-O-sulfate, gallic

acid, 3-O-methylgallic acid, 4-

methylgallic acid-3-O-sulfate,

protocatechuic acid, catechol glucuronide*, catechol sulphate* hydroxy-(hydroxyphenyl)pentenoic acid glucuronide*, hydroxy-

(hydroxy-methoxyphenyl)-pentenoic acid glucuronide I*, $^{*}$

hydroxy-(hydroxy-methoxyphenyl)pentenoic acid glucuronide $\|^{*}$, hydroxy-(hydroxyphenyl)pentenoic acid sulphate $\|^{*}$, hydroxy-(hydroxymethoxyphenyl)-pentenoic acid sulphate*, hydroxy-(dihydroxyphenyl) pentenoic acid sulphate*, hydroxy-

(hydroxyphenyl)pentenoic acid sulphate*, hydroxy-(dihydroxyphenyl) pentenoic acid glucuronide*, chlorogenic acid*,

1-methylpyrogallol-O-sulfate, 2methylpyrogallol-O-sulfate, pyrogallol-0-1-sulfate, pyrogallol-O-2-sulfate 2,4,6-trihydroxybenzaldehyde, 4-hydroxybenzaldehyde, Benzoylglutamic acid hippuric acid, 2-hydroxyhippuric acid,
Unspecific, common for many fruits and vegetables
Urine, plasma

Unspecific, common for many fruits and vegetables
References

$[49,50,12,2]$ 
Table 2 List of non-retained compounds for grapes/raisins and berries (Continued)

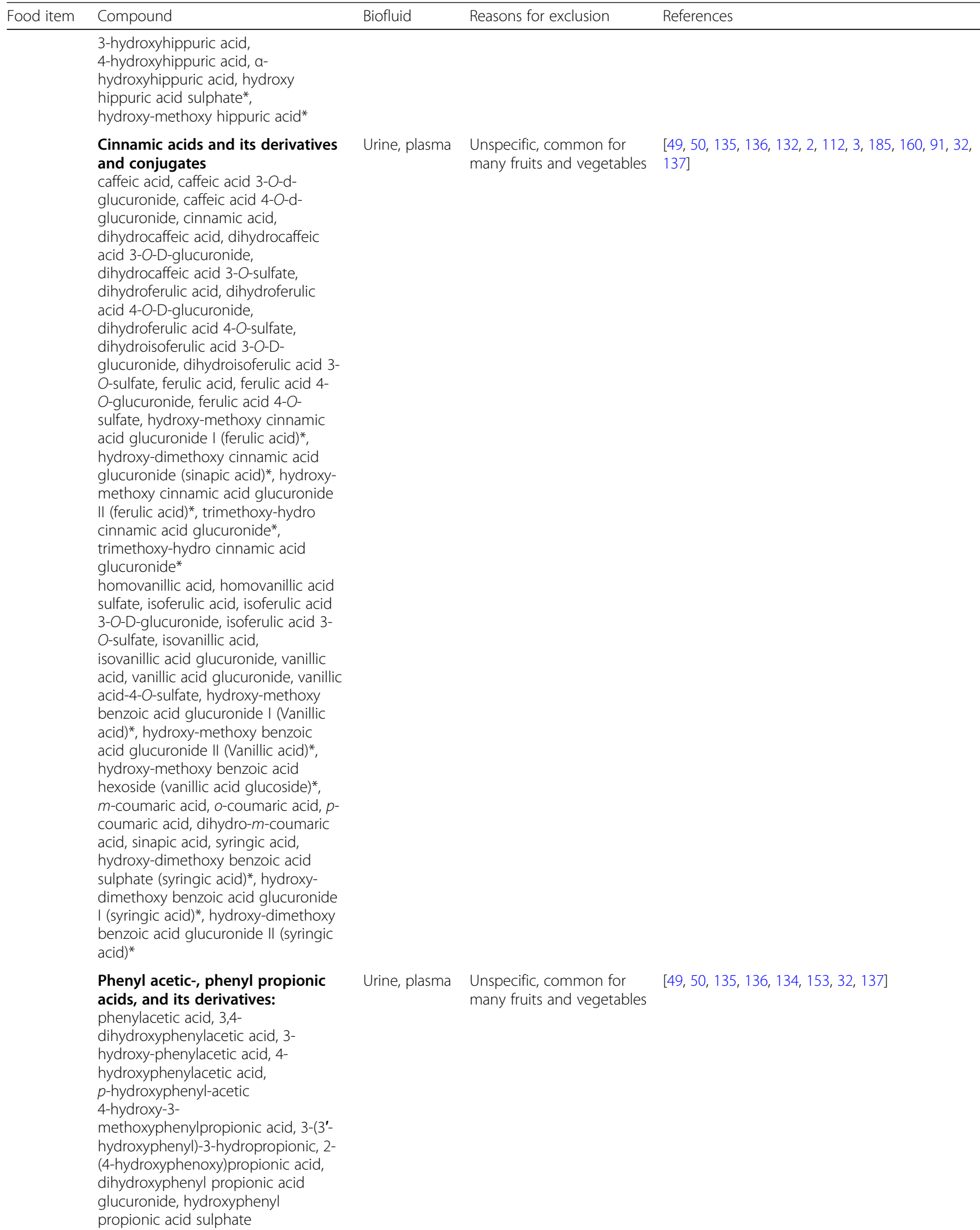


Table 2 List of non-retained compounds for grapes/raisins and berries (Continued)

\begin{tabular}{|c|c|c|c|c|}
\hline Food item & Compound & Biofluid & Reasons for exclusion & References \\
\hline & $\begin{array}{l}\text { Flavonols, its derivatives and } \\
\text { conjugates } \\
\text { kaempferol, kaempferol-3-O-D- } \\
\text { glucuronide, } \\
\text { quercetin, quercetin-3-O-D- } \\
\text { glucuronide } \\
\text { Methyl-dihydro myricetin* }\end{array}$ & Urine, plasma & $\begin{array}{l}\text { Unspecific, common for } \\
\text { many fruits and vegetables }\end{array}$ & {$[49,50,135,136,134,137,2]$} \\
\hline & $\begin{array}{l}\text { Flavan-3-ol metabolites } \\
\text { Catechin, epicatechin, (epi)catechin } \\
\text { glucuronide, (epi)catechin sulfate, } \\
\text { (epi)catechin glucuronide sulfate, } \\
\text { (epi)catechin methyl } \\
\text { glucuronide, (epi)catechin } \\
\text { methyl-sulfate }\end{array}$ & Urine, plasma & & [12] \\
\hline & $\begin{array}{l}\text { Abscisic acid*, hydroxy-abscisic acid } \\
\text { glucuronide*, hydroxy-abscisic acid } \\
\text { glucuronide*, hydroxy-abscisic acid* }\end{array}$ & Urine, serum & & {$[91,2]$} \\
\hline \multirow[t]{5}{*}{ Strawberry } & $\begin{array}{l}\text { Ellagic acids/ urolithins, its } \\
\text { derivatives and conjugates } \\
\text { ellagic acid, urolithin A, urolithin A } \\
\text { glucuronide, urolithin B, urolithin B } \\
\text { glucuronide }\end{array}$ & Urine, plasma & $\begin{array}{l}\text { Unspecific, common for } \\
\text { many fruits and vegetables }\end{array}$ & {$[9,14,25,26,74,129,165,148]$} \\
\hline & $\begin{array}{l}\text { Anthocyanidins, its derivatves } \\
\text { and conjugates } \\
\text { pelargonidin, pelargonidin- } \\
\text { 3-O-glucoside, pelargonidin- } \\
\text { 3-O-rutinoside, pelargonidin } \\
\text { glucuronide four isomers, } \\
\text { pelargodnidin sulfate, } \\
\text { 5-carboxypyranopelargonidin- } \\
\text { 3-o-beta-glucopyranoside, } \\
\text { cyanidin-3,5-diglucoside, cyanidin- } \\
\text { 3-O-glucoside, cyanidin-3-O-rutinoside }\end{array}$ & Urine, plasma & $\begin{array}{l}\text { Unspecific, common for } \\
\text { many fruits and vegetables, } \\
\text { low bioavailability }\end{array}$ & {$[4,5,22,41,48,73-76,113,126,147,179,20]$} \\
\hline & $\begin{array}{l}\text { Benzoic acids, its derivatives } \\
\text { and conjugates } \\
\text { 4-hydroxybenzoic acid, 3- } \\
\text { hydroxybenzoic acid, 3,4- } \\
\text { dihydroxybenzoic acid, gallic acid, } \\
\text { syringic acid, 4-hydroxyhippuric acid, } \\
\text { salicylic acid, syringic acid, gentisic } \\
\text { acid, vanillic acid, 3,4- } \\
\text { dihydroxybenzaldehyde, 4- } \\
\text { hydroxybenzaldehyde, }\end{array}$ & Urine, plasma & $\begin{array}{l}\text { Unspecific, common for } \\
\text { many fruits and vegetables }\end{array}$ & {$[4,5,129,145,148]$} \\
\hline & $\begin{array}{l}\text { Cinnamic acids, its derivatives } \\
\text { and conjugates } \\
\text { coumaric acid, caffeic acid, } \\
\text { homovanillic acid, ferulic acid, } \\
\text { synapic acid, vanillic acid, trans- } \\
\text { cinnamic acid, isovanillic acid }\end{array}$ & Urine, plasma & $\begin{array}{l}\text { Unspecific, common for } \\
\text { many fruits and vegetables }\end{array}$ & {$[4,5,14,145,148]$} \\
\hline & $\begin{array}{l}\text { Flavonols, its derivatives } \\
\text { and conjugates } \\
\text { fisetin, fisetin-3-rutinoside, } \\
\text { kaempferol, kaempferol-3- } \\
\text { (6"malonylglucoside), kaempferol-3- } \\
\text { coumaroylglucoside, kaempferol-3- } \\
\text { glucoside, kaempferol-3-O-sulfate, } \\
\text { kaempferol-glucuronide, dihydro } \\
\text { kaempferol glucuronide, kaempferol- } \\
\text { 3-coumaroyl-glucoside } \\
\text { quercetin, quercetin-3-(6"- } \\
\text { caffeoylgalactoside), quercetin-3- } \\
\text { galactoside, quercetin-3-glucoside, } \\
\text { quercetin-glucuronide, quercetin- } \\
\text { rutinoside, quercetin-sulfate, }\end{array}$ & Urine, plasma & $\begin{array}{l}\text { Unspecific, common for } \\
\text { many fruits and vegetables }\end{array}$ & {$[5,31,41]$} \\
\hline
\end{tabular}


Table 2 List of non-retained compounds for grapes/raisins and berries (Continued)

\begin{tabular}{ll}
\hline Food item $\quad$ Compound \\
\hline & methylquercetin, \\
& catechin \\
& hippuric acid \\
Raspberry & $\begin{array}{l}\text { Anthocyanidins, its derivatves and } \\
\text { conjugates } \\
\text { cyanidin-3-O-(2"-O-glucosyl)rutinoside, } \\
\text { cyanidin-3-O-(2"-O-xylosyl)rutinoside, } \\
\text { cyanidin-3-O-glucoside, cyanidin-3-O- } \\
\text { rutinoside, cyanidin-3-O-sophoroside, } \\
\text { cyanidin-3-O-glucuronide, } \\
\text { pelargonidin-3-O-(2"-O-glucosyl) } \\
\text { rutinoside, pelargonidin-3-O-gluco } \\
\text { side, pelargonidin-3-O-sophoroside, } \\
\text { peonidin-3-O-glucoside }\end{array}$ \\
\end{tabular}

Ellagic acids/ urolithins, its
derivatives and conjugates

ellagic acid, ellagic acid-O-

glucuronide, dimethylellagic acid-Oglucuronide, urolithin $A$, urolithin A-

O-glucuronide, urolithin A-sulfate, isourolithin A-O-glucuronide, uro

lithin $\mathrm{B}$, urolithin B-3-O-glucuronide,

(iso)urolithin A-sulfate-O-glucuronide

sanguiin $\mathrm{H} 6$

Cinnamic acids and its derivatives: caffeic acid, caffeic acid sulfate, caffeic acid-3'-sulfate, caffeoyl sulfate*, ferulic acid, ferulic acid-4'sulfate, ferulic acid-4'-O-glucuronide, isoferulic acid-3'-O-glucuronide, isoferulic acid-3'-sulfate, isoferulic acid-3'-O-glucuronide, dihydrocaffeic acid-3'-sulfate, dihydroferulic acid glucuronide, dihydroferulic acid sulfate

Benzoic acids and its derivatives: 3-hydroxybenzoic acid-4-sulfate, 4-hydroxybenzoic acid

4-hydroxybenzoic acid-3-sulfate,

2,6-dihydroxybenzoic acid,

2,3-dihydroxybenzoic acid,

2,4-dihydroxybenzoic acid, 3,4-

dihydroxybenzoic acid, methyl-4hydroxybenzoic acid, methyl-3,4-

dihydroxybenzoic acid, 4-

hydroxybenzoic acid glucuronide,

3-hydroxybenzoic acid glucuronide, gallic acid hippuric acid,

4-hydroxyhippuric acid, 3-

hydroxyhippuric acid,

3,4-dihydroxybenzoic acid sulfate

3,4-dihydroxybenzaldehyde, 4hydroxybenzaldehyde glucuronide, 4-hydroxybenzaldehyde

Phenyl acetic-, phenyl propionic acids, and its derivatives:

3,4-dihydroxyphenyl acetic acid, 3'methoxy-4'-hydroxyphenylacetic acid, 3,4-dihydroxyphenylacetic, 4-hydroxyphenylacetic acid,

Biofluid Reasons for exclusion

References

Plasma Unspecific, common for many fruits and vegetables

Plasma

Unspecific, common for many fruits and vegetables

Urine, plasma ileal fluid

Unspecific, common for many fruits and vegetables, low bioavailability

$[70,98,184]$ low bioavailability

Urine, plasma

Unspecific "common for

$[70,98,184]$ many fruits and nuts

Ileal fluid

low bioavailability, low concentrations in biological fluids

Urine, plasma Unspecific, common for many fruits and vegetables

$[95,98,184]$

Urine, plasma

Unspecific, common for many fruits and vegetables

$[95,98,184]$
Urine, plasma

Unspecific, common for many fruits and vegetables
$[98,184]$ 
Table 2 List of non-retained compounds for grapes/raisins and berries (Continued)

\begin{tabular}{l} 
Food item $\quad$ Compound \\
\hline 3-hydroxyphenylacetic acid, \\
2-hydroxyphenylacetic acid, 3- \\
hydroxyphenylpropionic acid sulfate, \\
3-hydroxyphenylpropionic acid \\
Vitamin C \\
naringenin glucuronide \\
methyl-epicatechin sulfate* \\
catechin glucuronide \\
Endogenous metabolites: \\
indoxyl sulfate, adenine, alanine, \\
asparagine, betaine, carnitine, citrate, \\
formate, glutamine, glycine, histidine, \\
lysine, N-phenylacetylglycine, \\
phenylacetate, serine
\end{tabular}

\section{Blackberry Anthocyanins and its derivatives:}

cyanidin 3-glucoside, cyanidin 3xyloside, cyanidin glucuronide, cyanidin diglucuronide, cyanidin sulfate, 4'-methyl-cyanidin3-glucoside, cyanidin 3-glucoside, cyanidin 3-rutinoside, cyanidin glucoside glucuronide, cyanidin glucoside sulfate, cyanidin glucuronide (two isomers), methyl cyanidin glucoside glucuronide, methyl cyanidin glucuronide, cyanidin-3-rutinoside, cyanidin-3-xylosylrutinoside, cyanidin-3-glucoside, cyanidin-3sambubioside, cyanidin 3-rutinoside, cyanidin 3-sambubioside, cyanidin 3xylosylrutinoside, cyanidin-3-Oglucoside, methyl cyanidin 3glucoside, methyl cyanidin 3rutinoside, methyl cyanidin 3sambubioside, methyl cyanidin 3-xylosylrutinoside, peonidin-3-glucoside, peonidin 3xyloside, peonidin glucuronide, peonidin-3-glucoside glucuronide, 3' methyl-cyanidin-3-glucoside

\section{Ellagic acids/ urolithins, and} its derivatives:

ellagic acid, urolithin $A$, urolithin $A$ glucuronide, urolithin $B$, urolithin $B$ glucuronide, urolithin $C$, urolithin C glucuronide, urolithin $D$, urolithin $D$ glucuronide, urolithin M5, methyl urolithin A, ellagic acid dimethyl ether glucuronide, ellagic acid dimethyl ether, hexahydroxydiphenyl

Others metabolites (2,3-

dihydroxyisovaleric acid, 2,5furandicarboxylic acid, 2

isopropylmalate, 2-oxindole-3acetate, 3-[3-(sulfooxy)phenyl] propanoic acid, 3hydroxyphenylacetic acid, 3methylhistidine, 4-acetylphenol sulfate, 5-hydroxymethyl-2-furoic acid, abscisic acid, anthranilate,

Biofluid Reasons for exclusion

References

$\begin{array}{lll}\text { Urine } & \begin{array}{l}\text { Unspecific, common for } \\ \text { many fruits and vegetables }\end{array} & {[95]} \\ \text { Urine } & \begin{array}{l}\text { Unspecific, common for } \\ \text { many fruits }\end{array} & {[95]} \\ \text { Urine } & \begin{array}{l}\text { Unspecific, common for } \\ \text { many fruits and vegetables }\end{array} & {[95,184]} \\ \text { Plasma } & \text { Endogenous metabolites } & {[86]}\end{array}$

Urine, plasma

Unspecific, common for many fruits and vegetables

$[47,100,123,161]$
Spot urine

Unspecific, common for many fruits and nuts

Unspecific, common for many fruits and vegetables
$[64,65]$ 
Table 2 List of non-retained compounds for grapes/raisins and berries (Continued)

\begin{tabular}{l} 
Food item Compound \\
\hline chiro-inositol, cinnamoylglycine, \\
cis-aconitate, cortisol, cortisone, \\
methyl-beta-glucopyranoside, $n$-(2- \\
furoyl)glycine, N-acety- \\
3-methylhistindine, N-acetylproline, \\
xylitol, xylose, glycerol 3-phosphate \\
Benzoic acids, and its derivatives: \\
4-methylcatechol sulfate, catechol, \\
catechol sulfate, \\
hippuric acid, 3-hydroxyhippuric acid, \\
4-hydrixyhippuric acid, \\
2,4,6-trihydroxybenzoic acid
\end{tabular}

Cranberry Anthocyanins and its derivatives: cyanidin-3-arabinoside, cyanidin-3galactoside, cyanidin-3-glucoside, malvidine-3-glucoside, peonidin-3-arabinoside, peonidin-3galactoside, peonidin-3-glucoside

Cinnamic acids and its derivatives: ferulic acid, ferulic acid sulfate*, dihydro ferulic acid, dihydro ferulic acid 4-O-D-glucuronide, dihydro ferulic acid 4-O-sulfate, dihydro isoferulic acid 3-O-D-glucuronide, dihydro isoferulic acid 3-O-sulfate, ferulic acid 4-O-glucuronide, ferulic acid 4-O-sulfate, isoferulic acid, isoferulic acid 3-O-D-glucuronide, isoferulic acid 3-O-sulfate, coumaric acid sulfate*, $m$-coumaric acid, o-coumaric acid, p-coumaric acid, cinnamic acid, 3,4-

dihydroxyhydrocinnamic acid*, dihydroxyhydrocinnamic acid-3-Oglucuronide* caffeic acid, caffeic acid 3-O-Dglucuronide, caffeic acid 4-O-Dglucuronide, dihydro caffeic acid, dihydro caffeic acid 3-O-Dglucuronide, dihydro caffeic acid 3O-sulfate, dihydro caffeic acid 4-0sulfate, vanillic acid, vanillic acid-4-Osulfate, homovanillic acid, homovanillic acid sulfate, isovanillic acid, sinapic acid*, sinapic acid glucuronide, syringic acid,

Flavonols and its derivatives: kaempferol, kaempferol-3-O-Dglucuronide, quercetin, quercetin-3O-D-glucuronide, quercetin 3galactose, quercetin 3-rhaminoside, quercetin 3 -arabinose myricetin, myricetin 3-arabinose, myricetin 3-galactoside epicatechin, isorhamnetin,

Benzoic acids, and its derivatives: benzoic acid, 2-hydroxybenzoic acid, 3-hydroxybenzoic acid, 4hydroxybenzoic acid, 2,3dihydroxybenzoic acid, 2,4dihydroxybenzoic acid, 2,5dihydroxybenzoic acid, trihydroxybenzoic acid, quinic acid, 4-methylgallic acid-3-O-

Biofluid Reasons for exclusion

References

Urine, plasma

Unspecific, common for many fruits and vegetables

Urine, plasma

Unspecific, common for many fruits

Urine, plasma Unspecific, common for many fruits and vegetables
$[64,65,123]$

[108] [119];

$[94,135,136,48-51,174,175,183,27,7]$
Urine, plasma
Unspecific, common for many fruits and vegetables
$[135,136,48-51,174,175,27]$
Unspecific, common for many fruits and vegetables
$[94,135,136,48-51,174,175,183,27,7]$ 
Table 2 List of non-retained compounds for grapes/raisins and berries (Continued)

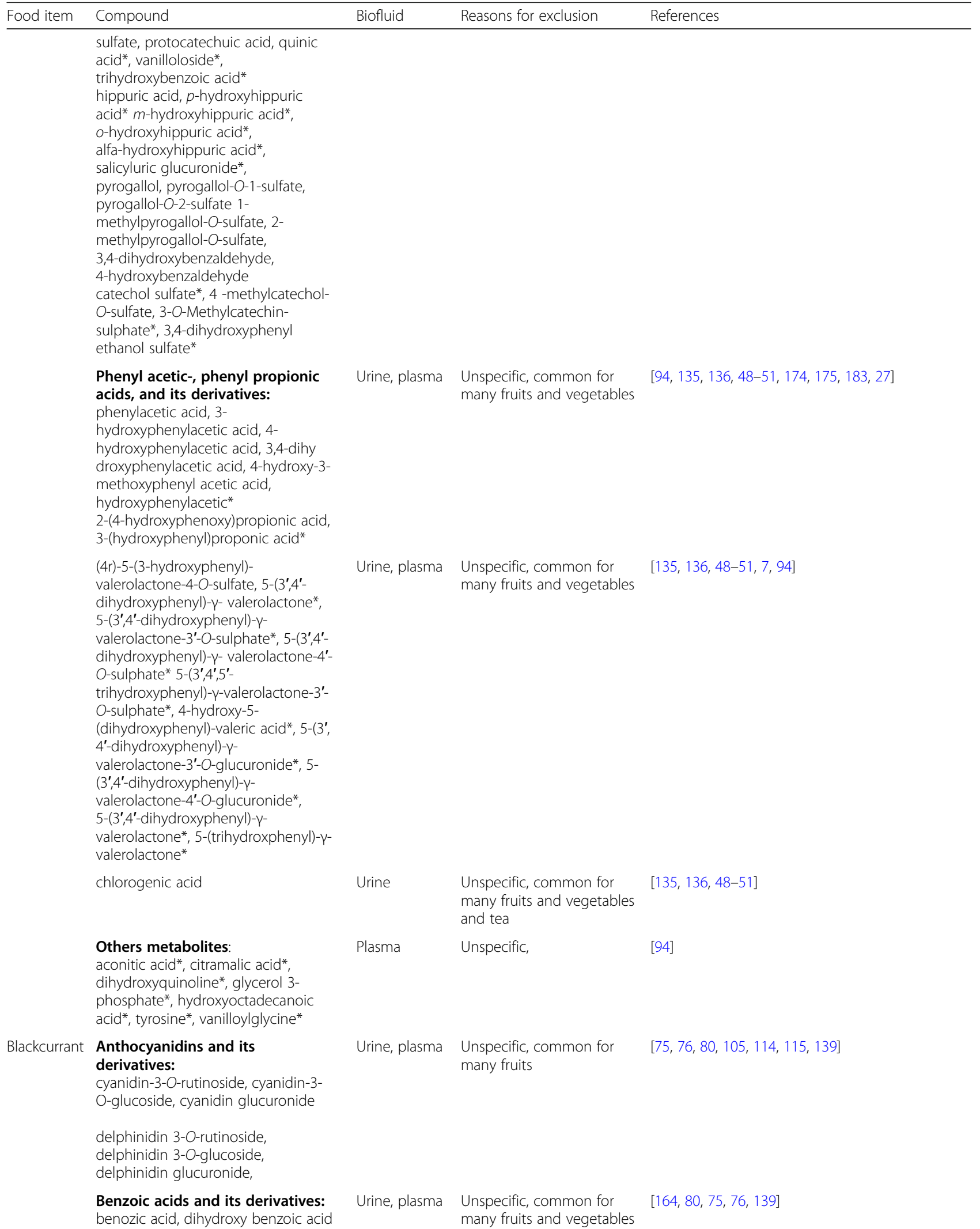


Table 2 List of non-retained compounds for grapes/raisins and berries (Continued)

\begin{tabular}{|c|c|c|c|c|}
\hline Food item & Compound & Biofluid & Reasons for exclusion & References \\
\hline & \multicolumn{4}{|l|}{$\begin{array}{l}\text { acid, 4-hydroxyhippuric acid, cat } \\
\text { echol sulfate* }\end{array}$} \\
\hline & $\begin{array}{l}\text { Cinnamic acids and its derivatives: } \\
\text { dimethoxy cinnamic acid sulfate*, } \\
\text { ferulic acid, ferulic acid sulfate* }\end{array}$ & Urine, plasma & $\begin{array}{l}\text { Unspecific, common for } \\
\text { many fruits and vegetables }\end{array}$ & {$[80,164]$} \\
\hline & \multicolumn{4}{|l|}{ caffeic acid sulfate* } \\
\hline & $\begin{array}{l}\text { Phenylacetic acid and its } \\
\text { derivatives: } \\
\text { dihydroxy phenylacetic acid sulfate* }\end{array}$ & Urine & $\begin{array}{l}\text { Unspecific, common for } \\
\text { many fruits and vegetables }\end{array}$ & [164] \\
\hline & Quercetin & Plasma & $\begin{array}{l}\text { Unspecific, common for } \\
\text { many fruits and vegetables }\end{array}$ & [44] \\
\hline & Vitamin C & Plasma & Unspecific & [80] \\
\hline & uric acid & Plasma & $\begin{array}{l}\text { Unspecific, endogenous } \\
\text { compound }\end{array}$ & {$[80]$} \\
\hline
\end{tabular}

* Metabolites found using untargeted metabolomics

urolithin D, and urolithin M5. Additionally, three studies included glucuronide and sulfate conjugates of urolithins $([25,26,98] \mathrm{A}, \mathrm{B},[165])$. A reason for considering urolithins as putative BFIs for berries is the presence of ellagitanins (from several hundreds of $\mathrm{mg} / \mathrm{kg}$ to $>1 \mathrm{~g} / \mathrm{kg}$ ) in strawberries, raspberries, and blackberries, with a limited number of other food sources which could interfere.

We examined the relevance of these compounds and others as putative BFIs for every type of berry.

\section{Blueberries}

Eight studies investigated anthocyanin bioavailability after consumption of blueberries in different forms: frozen blueberries, fresh bluberries, blueberry extract, blanched and unblanched blueberries, blueberry juice, and freeze dried berries [37, 81, 82, 103, 105, 112, $178,185]$. The major anthocyanins in blueberries are glycosides of delphinidin and malvidin (Supplementary Table 1). In most studies, the anthocyanin profile in plasma or urine matched the composition of the ingested blueberry. Three malvidin glycosides were the dominant urine anthocyanins in the study by McGhie et al. [105]. Mazza et al. [103] and Del Bo et al. [36] reported malvidin-3-galactoside and malvidin-3-glucoside as the most concentrated metabolites in serum, followed by delphinidin-3-glucoside and petunidin-3-glucoside, this pattern perfectly reflecting the composition of the blueberry extract used in the study. Zhong et al. [185] found delphinidin glucuronide and dephinidin glucoside followed by malvidin glucoside and petunidin glucoside as major metabolites in urine. Unexpectedly, Kalt et al. [81-83] found pelargonidin glucuronide being dominant with delphinidin glucuronide in 24-h urine. As the blueberry juice used was devoid of pelargonidin aglycone or glycosides, the authors suggested that the presence of pelargonidin metabolites may be explained by an interconversion between anthocyanins. Although the detection of anthocyanins may indicate the consumption of certain berries, their low concentrations as well as their chemical instability appear to drastically limit their use as quantitative BFIs, and most likely also qualitative use.

Not only were anthocyanins found in biological fluids, but also a number of phenolic compounds, some of them being anthocyanin microbial metabolites [3, 12, 32, $50,53,112,134,136,137,153,160]$. In the study by Feliciano et al. [50], a total of 61 phenolic metabolites were quantified in plasma at baseline, of which 43 increased after acute or 1-month supplementation with blueberry drinks. Benzoic and catechol derivatives represented more than $80 \%$ of the changes in the phenolic profile after $2 \mathrm{~h}$ consumption on day 1 , whereas hippuric and benzoic derivatives were the main compounds that increased at 0 and $2 \mathrm{~h}$, respectively on day 30 . All of these metabolites, however, are common for a wide range of fruits and vegetables, and therefore cannot be considered as specific biomarkers of blueberry consumption.

Two studies used an untargeted approach aiming at exploring the metabolome changes after blueberry intake [2, 91]. Ancillotti et al. reported a high number of plasma and urine metabolites that were statistically significant after consumption of a single 25-g dose of Vaccinum myrtillus. The authors applied a robust analysis of MS/MS patterns for a reliable identification. However, they did not find metabolites specific enough for blueberry to serve as candidate BFI. Among the most discriminant metabolites were benzoic, ferulic, and phenylpropionic acid derivatives, as well as phenylvaleric acid and phenyl-valerolactone derivatives. 
Additionally, two anthocyanins, delphinidin hexoside and cyanidin hexoside, were detected in urine. Langer et al. similarly attempted to identify blueberry-derived metabolites in plasma and urine metabolomes $2 \mathrm{~h}$ after intake of fresh blueberries or juice. However, compound identifications were only putative, without acquisition of MS-MS spectra, and mostly non-specific phenolic acids were reported.

Based on the available studies, it is hard to propose any candidate BFI for blueberry. The presence of malvidin and its phase II metabolites in urine, together with other anthocyanins, may indicate blueberry intake. However, they are obviously not specific and robust enough to constitute a BFI. Another limitation is the very low concentration of these metabolites in biological fluids, frequently below the limit of detection. Further studies should definitely be undertaken in humans, considering other components than anthocyanins in blueberries. Application of untargeted metabolomics with a reliable and robust annotation process, may bring new insights on unforeseen markers of blueberry intake. In particular, the increasing availability of chemical standards, the enrichment of spectral libraries and new bioinformatics tools allowing accurate prediction of metabolism, and MS fragmentation will help in the future to improve the annotation of discriminating compounds, and possibly reveal unforeseen candidate BFIs for blueberry.

\section{Cranberries}

Cranberry is reported to have health benefits, including prevention of urinary tract infections, due to its high content in phytochemicals, including proanthocyadinins $\mathrm{A}$ and other polyphenols [54, 88, 125]. Although these health benefits have been recognized, a very limited number of studies have investigated the circulation of cranberry-derived metabolites in humans [7, 27, 49, 94, 108, 119, 175]. Among the publications included in this review, some focused on the analysis of structurally related anthocyanin metabolites $[27,108,119]$, which are present in very low amounts in plasma in comparison to their phenolic acid metabolites, while others quantified small phenolic acids after cranberry intake $[49,51,135,174,175,183]$. Interestingly, two studies used an untargeted approach to investigate changes in plasma and urine metabolome after consumption of cranberry juice [7, 94].

Four studies have focused on targeted analysis of anthocyanins and flavonols [27, 108, 119, 175]. Seven anthocyanins were found in plasma at low nanomole per litre levels after consumption of a single dose of $480 \mathrm{~mL}$ of cranberry juice, namely peonidin-3-O-galactoside > cyanidin-3-O-arabinoside $>$ cyanidin-3-O-galctoside $>$ peonidin-3-O-arabinoside $>$ cyanidin-3-O-glucoside $>$ peonidin-3-O-glucoside $>$ malvidin-3-O-glucoside [108] . The pattern of anthocyanin glucosides observed in urine was similar to that in plasma, with the exception of malvidin-3-O-glucoside, and generally reflected the relative concentrations present in the juice or the cranberry extract beverage $[27,108,119]$.

One study aimed at verifying whether proanthocyanin dimer A-2 could be a valid biomarker of cranberry intake. A progressive, weekly dosing schedule protocol was applied, involving 5 healthy females drinking for 7 weeks a cranberry juice at increasing frequencies [174]. While proanthocyanin dimer A-2 was quantifiable in urine, it did not appear to be excreted in a concentration that corresponded to the dosing schedule and intake of cranberry juice. Additionally, proanthocyanin dimer A-2 was not detected in all spot and 24-h urine samples of the three subjects. These data suggest that proanthocyanin dimer A-2 cannot be considered as a good candidate intake biomarker. Liu et al. [94] studied metabolome changes in plasma after cranberry juice intake, compared with apple juice intake, assuming that different types of procyanidins (A-type vs B-type, respectively) from the two juices can differently impact the human metabolome. Through the untargeted assay, several endogenous and exogenous metabolites were found to discriminate the two interventions. Among the metabolites differentiating cranberry intake from apple intake were quinic acid, catechol sulfate, 3,4-dihydroxyphenyl ethanol sulfate, coumaric acid sulfate, ferulic acid sulfate, citramalic acid, hydroxyoctadecanoic acid, hippuric acid, 2hydroxyhippuric acid, and vanilloylglycine (Table 2). Although among the annotated metabolites, none seem specific to cranberry, several discriminant features remained unidentified. Further efforts for more extensive and reliable annotation are required, to ensure full structure elucidation of all highly discriminant metabolites. Baron et al. [7] used a mixed untargeted and targeted approach to analyze urine samples from a randomized, double-blind, 2-arm repeated measure cross-over study with cranberry extract. Again, only non-specific small phenolics and valerolactones conjugated to sulfate and glucuronide moieties were identified among the discrimimant features (Table 2).

A wide range of different classes of phenolic acids and their sulfate and glucuronide conjugates have been quantified in plasma and urine after cranberry intake [49, 51, 127, 135, 175, 183]. Rodriguez-Mateos et al. [135] investigated the absorption, metabolism, and excretion of cranberry polyphenols in the plasma and urine of healthy young men after the consumption of $450 \mathrm{~mL}$ of cranberry juice, providing an increasing dose of total polyphenols: $409 \mathrm{mg}, 787 \mathrm{mg}, 1238 \mathrm{mg}, 1534 \mathrm{mg}$, and $1910 \mathrm{mg}$. In plasma, hippuric acid and catechol-O-sulfate were found at the highest concentrations, followed by 2 , 3-dihydroxybenzoic acid, phenylacetic acid, and others (see Table 2). The metabolite profile found in urine was 
very similar, with hippuric acid representing $64 \%$ of the excreted metabolites, while $14 \%$ were phenylacetic acids, followed by benzoic acids (9\%), catechols (5\%), valerolactones (4\%), and cinnamic acids (3\%). Total plasma, but not urinary excreted (poly)phenol metabolites, exhibited a linear dose response, driven by caffeic acid 4-O-ß-Dglucuronide, ferulic acid derivatives, and benzoic acid derivatives (see Table 2).

Recently, terpenes have gained considerable attention, being found at significant concentrations in cranberries [87]. Ursolic acid, a ubiquitous triterpenoid, was found as the dominant terpene in American cranberries (46-109 $\mathrm{mg} / 100 \mathrm{~g} \mathrm{FW})$, together with two other rare derivatives of ursolic acid: cis-3-O-p-hydroxycinnamoyl ursolic acid (12-16 mg/100 g FW) and trans-3-O-p-hydroxycinnamoyl ursolic acid (42-60 mg/100 g FW) [87]. Monotropein, 6,7dihydromonotropein, and other iridoid derivatives have also been described in cranberry [16]. More effort should be put on performance of the untargeted experiments, which have the potential to reveal new BFIs.

\section{Blackcurrant}

Blackcurrant phytochemicals have been found to offer a variety of beneficial effects, including immunomodulatory, antimicrobial, and anti-inflammatory actions and inhibition of low-density lipoprotein, while the high vitamin $C$ content of blackcurrant $(18-103 \mathrm{mg} / \mathrm{g}$ fresh weight) can help to achieve the recommended daily intake [56, 73, 77, 80, 109, 124, 130]. However, little is known about the recovery of blackcurrant metabolites in biofluids and no attempt to identify BFI has been made so far. Only a few studies have investigated the bioavailability of blackcurrant anthocyanins, which is of little relevance for identifying candidate BFIs. Two anthocyanins, namely delphinidin-3-rutinoside and cyanidin-3-rutinoside were reported in urine samples collected $2-4 \mathrm{~h}$ postprandially $([76,115]$; and [80]. Only one study reported anthocyanins in plasma Nakamura et al. [114] quantified the following compounds in decreasing order of concentration: delphinidin-3-rutinoside $>$ cyanidin-3-rutinoside $>$ delphinidin-3-glucoside $>$ cyanidin3 -glucoside, which was consistent with the respective quantities of the ingested anthocyanins (see Table 1 for details). Blackurrants have an interesting aroma profile with monoterpenoids being particularly high such as 3-carene, sabinene, humulene, $\alpha$ pinene, and terpinolene $[17,39,52]$ (see Supplementary Table 1). However, there are no investigations yet on the metabolism of aroma blackcurrant in humans. There is thus a critical need for data related to the impact of blackcurrant on the human metabolome, and for identification of blackcurrant metabolites that could act as potential biomarkers of intake.

\section{Strawberries}

Of the twenty investigations included for this review for strawberry, most used a targeted metabolomics approach to detect the low concentrations of anthocyanins in biological fluids, with the exception of one study [31], where an untargeted approach was used. As regards strawberry composition, pelargonidin-based anthocyanins and procyanidins are particularly high (see Supplementary Table 1) but not specific to strawberry, while furaneol and mesifurane are characteristic aroma compounds of strawberry.

Several studies reported pelargonidin metabolites in the urine metabolic profile after strawberry intake $[4,20,22$, $113,126]$. Mullen et al. identified four pelargonidin glucuronide isomers, pelargonidin-3-O-glucoside and pelargonidin aglycone in plasma and urine, while pelargonidin sulfate was detected only in urine. Similar profiles were found in urine by Carkeet et al. [22], Azzini et al. [4], Park et al. [126], Felgines et al. [48], Hollands et al. [75], and [20]. Three investigations considered dose-response effects $[22,48,75]$ and reported that urinary excretion of pelargonidin-3-glucoside and pelargonidin glucuronide isomers increased linearly with the dose of strawberry. However, the total urinary excretion of strawberry anthocyanin metabolites remained low corresponding to only $0.9-1.80 \%$ of the pelargonidin-3-glucoside ingested.

The profiles of pelargonidin metabolites in plasma were similar to those in urine $[5,41,113,179]$. Banaszewski et al. [5] characterized the postprandial plasma profile of strawberry anthocyanins, particularly pelargonidin-based anthocyanins, across 4 doses using targeted and untargeted analysis. Pelargonidin-O-glucuronide was found to be the primary pelargonidin metabolite and showed a dose-response relationship. However, while the maximum concentration in plasma and the area under the concentration-time curve increased with the dose, the concentrations decreased as the percentage of the dose, suggesting a possible saturation of the absorptive mechanisms or an increased efficiency in elimination. Apart from the glucuronide conjugate, qualitative Q-TOF LC/MS analysis revealed 33 other phenolic compounds, including a wide range of pelargonidin metabolites, such as pelargonidin sulfate, pelargonidin-3-O6" '-rhamnosylglucoside, carboxypyranopelargonidin-3-Oglucopyranoside, pelargonidin-3-(6"'-caffeoylglucoside), pelargonidin-3,5-diglucoside, pelargonidin-3-sambubioside, and pelargonidin-3-(6"'-malonylglucoside). Similarly, Edirisinghe et al. [41], who analyzed plasma using targeted LC-MS/MS, detected pelargonidin sulfate, pelargonidin-3$\mathrm{O}$-glucoside, and pelargonidin-3-O-glucuronide to be the most abundant metabolites following intake of a single portion of strawberries. Quantification of pelargonidin sulfate, pelargonidin glucoside and pelargonidin glucuronide showed concentrations in the low nanomole per litre range, with $\mathrm{T}_{\max }$ varying between 1.1 and $2 \mathrm{~h}[41,113,147]$. 
Urolithins have been the subject of investigations by several authors $[9,14,25,26,129,148,165]$, and their appearance in biological fluids was studied after strawberry intake in chronic and acute modes. In an acute study [25], the microbial metabolite urolithin B glucuronide was first detected along the urine fractions from 32 to $56 \mathrm{~h}$. Considerable inter-individual differences, probably associated with differences in colonic microbiota composition, were noted, identifying "high- and lowmetabolite excreters". Later, Truchado et al. [165] found urolithins in urine profiles after fresh strawberry intake, with urolithin A glucuronide as the predominant metabolite, followed by urolithin A, urolithin B, and urolithin $B$ glucuronide. All urolithins appeared in urine within a time ranging from 8 to $32 \mathrm{~h}$. Urolithin B and urolithin B glucuronide were detected in only 4 volunteers ( $20 \%$ of the total population), consistently with the reported fact that only part of the population synthesizes urolithin B; actually, only part of the population has the microbiota species that are able to realize all metabolic steps required to convert ellagitannins/ellagic acid into urolithin B. The mean urinary excretion of urolithin conjugates over $92 \mathrm{~h}$ reached $58 \pm 48 \%$ of the amount of total ellagic acid administered $(62 \mathrm{mg})$ after fresh strawberries and $57 \pm 52 \%$ after strawberry puree. On the other hand, no urolithin A was found in plasma after several days of strawberry consumption in two studies [14, 129], and only trace amounts of ellagic acid $(15.2 \pm 5.2 \mathrm{ng} / \mathrm{mL})$ were detected by Basu et al. [9]. Urolithins present several limitations as candidate BFIs. Walnuts and other fruits contain ellagitannins; thus, the specificity for strawberry is quite low, although strawberries are a very rich source of both simple and complex ellagitannins [67], and among the fruit containing ellagitannins, strawberries are the most widely consumed. Their main ellagitannin, agrimoniin, has been suggested to be one of the most widely present ellagitannins in the human diet due to strawberry intake [172], while casuarictin is the second characteristic strawberry ellagitannin [67]. The interindividual variation in urolithin production by the microbiota is also likely to affect their accuracy as quantitative BFIs. However, the interest of total urolithins as components of a multi-marker BFI for strawberries deserve further investigation, especially in observational setting.

Three studies suggested the importance of strawberry aroma compounds in the search for candidate BFIs. One untargeted experiment was performed by Cuparencu et al. [31], in which three furaneol and mesifurane metabolites were found in urine, namely furaneol glucuronide, furaneol sulfate, and mesifurane sulfate. This randomized controlled crossover meal study was designed to study urine kinetics after a single intake of $150 \mathrm{~g}$ of strawberry puree. Furaneol metabolites had already been the subject of investigation in 1997 by Roscher et al. [141], who reported furaneol glucuronide to be the main strawberry aroma metabolite in urine. In 2010, Henning et al. [74] quantified furaneol glucuronide using LC-MS/MS in both plasma and urine of 21 volunteers receiving $250 \mathrm{~g}$ of strawberries for 3 weeks. Plasma concentrations at $6 \mathrm{~h}$ were $148.9 \pm 223.8 \mathrm{nM}$, while in 0 12-h urine, $14.5 \mu \mathrm{mol} / \mathrm{g}$ creatinine were detected. Although fairly specific for strawberry, aroma metabolites have still not gained much attention as intake biomarkers. Furaneol was also found in pineapples, raspberries, or kiwi fruits; moreover, it is a product of Maillard reactions [151], which indicate cooking processes such as baking or frying. Therefore furaneol derivatives alone can hardly be proposed as BFI for strawberry intake.

In terms of biomarker validation criteria, pelargonidin and its conjugates, as well as utolithins achieve plausibility criterion, however fail at specificity. Aroma metabolites seem to be plausible and specific; however, there is a lack of information related to the concentration in biological fluids. Definitely more studies are required to understand ADME processes of aroma compounds in humans. To summarize, based on current knowledge, only a multi-metabolite biomarker panel could be suggested to indicate strawberry intake (see Fig. 2 with

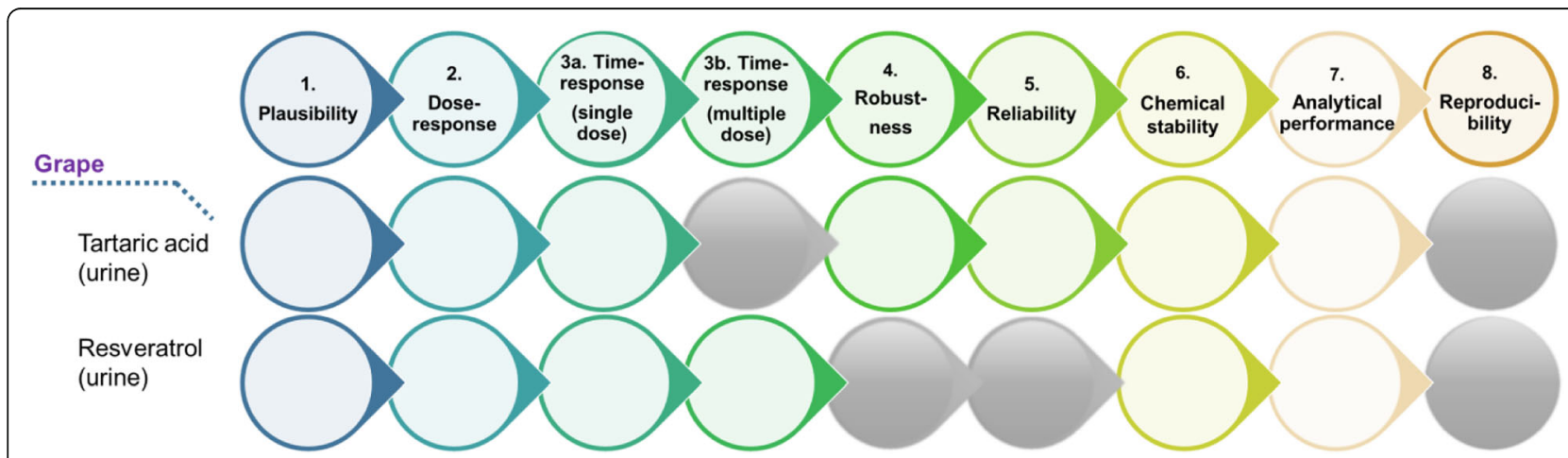

Fig. 2 Overview of the validation process and its application on candidate BFIs for grape 
validation scheme). Pelargonidin glucuronide together with urolithins and furaneol/mesifuran derivatives seem the most appropriate candidates; however, differences in excretion rates requires an intensive work to identify the most applicable biofluid and time for sample collection.

\section{Raspberries}

Raspberries have a phytochemical profile also rich in ellagitannins and anthocyanins, with sanguiin H-6 and lambertianin $\mathrm{C}$, as well as cyanidin-3-O-sophoroside, cyanidin-3-O-(2-O-glucosyl)rutinoside and cyanidin-3$O$-glucoside, being the most characteristic compounds. The specific aroma of raspberries is mainly due to the presence of 4-( $p$-hydroxyphenyl)-2-butanone, commonly called raspberry ketone.

There are no human studies considering the metabolism of raspberry aroma compounds, although such compounds seem to be very specific. The metabolism of raspberry ketones has been investigated in animals only: rats, guinea pigs, and rabbits [156]. Raspberry ketone was rapidly absorbed from the gastrointestinal tract after administration of a single dose of $1 \mathrm{mmol} / \mathrm{kg}(164 \mathrm{mg} / \mathrm{kg}$ bw) by oral gavage. The majority of ingested raspberry ketone was excreted in urine within the first $24 \mathrm{~h}$ as its phase II conjugated metabolites (glucuronide and sulfate forms). In addition to raspberry ketone phase II metabolites, a total of 14 other compounds were detected in urine. Of these, the reduction product 4-(4-hydroxyphenyl)butan-2-ol was the most abundant, followed by 4hydroxyphenylacetic acid, 2-(4-hydroxyphenyl)ethanol, 4-(3,4-dihydroxyphenyl)butan-2-one, and 4-(4-hydroxyphenyl)butan-2,3-diol. Total urinary recovery was close to $90 \%$ for the three animal species. Considering that raspberry ketone is specific to the raspberry and seems to be well absorbed, more effort is obviously required in the future to determine and characterize the metabolites of this particular aroma compound in human biological fluids. However, raspberry ketone is increasingly used as a food additive due to its pleasant flavour. Thus, its detection would have to be accompanied by other raspberry metabolites to reflect berry intake and not consumption of aromatized foods.

Metabolites of raspberry ellagitannins and anthocyanins have been the focus of investigations by several groups [70, 78, 98, 184]. In the study by Ludwig et al. [98], a total of 27 metabolites were detected in urine for over a 48-h collection period after a single intake of 300 $\mathrm{g}$ of raspberry, and 15 metabolites were found in plasma for over a 24-h collection period. Cumulative urine metabolite profile was dominated by derivatives of phenylpropanoic acid, urolithins, and ellagic acid, while mainly urolithins and ellagic acid derivatives were detected in plasma. The profiles of urolithin metabolites in both matrices were very similar, with urolithin A-sulfate as the dominant metabolite, followed by urolithin A-O-glucuronide, urolithin B-3-O-glucuronide, (iso)urolithin Asulfate-O-glucuronide, isourolithin A-O-glucuronide, dimethylellagic acid-O-glucuronide, characterized by $\mathrm{T}_{\text {max }}$ between 8 and $24 \mathrm{~h}$ and concentrations ranging between 3.5 and $450 \mathrm{nmol} / \mathrm{L}$. The presence of cyanidin-3$O$-glucoside in urine and plasma after raspberry intake was found at low nanomoles per litre levels by the three authors (González-Barrio et al. [70], Ludwig et al. [98], and Istas et al. [78]), confirming that native anthocyanins occur only at low trace amounts in biological fluids.

The other metabolites reported in urine and plasma after raspberry intake included mainly small phenolic acids belonging to benzoic, phenyl acetic, and hippuric acid derivatives, as well as ferulic and caffeic acid derivatives, ascorbic acid, and methylepicatechin sulfate. All these metabolites, however, occur frequently in urine after different nutritional interventions with plant foods, therefore cannot be considered as candidate BFIs for raspberry even as part of a multi-marker [78, 95, 98, 184].

There is a considerable need for more human studies focusing on raspberry intake. It might be speculated that raspberry ketone metabolites together with total urolithin metabolites in urine samples could be considered as potential candidates of indicative markers, or act as multi-metabolite biomarker panel; however, the topic remains widely open.

\section{Blackberries}

In the case of blackberries, investigations included in this review also mostly considered metabolites of anthocyanins and ellagitanins. Three studies were single-dose experiments involving healthy volunteers $[47,64,100]$, and in all of them, a metabolomics approach targeted on cyanidins and urolithins was applied for plasma and urine profiling.

Cyanidin metabolites were found in biological fluids by Felgines et al. [47], García-Muñoz et al. [64, 65], and Marques et al. [100]. In Marques' study, methyl cyanidin glucuronide and 3'methyl-cyanidin-3-glucoside were the main anthocyanin conjugates detected in both plasma and urine samples. Apart from these metabolites, other minor compounds were detected: cyanidin glucuronide, cyanidin glucoside sulfate, methyl cyanidin glucoside, cyanidin-3-glucoside-7-glucuronide, cyanidin rutinoside, and cyanidin-3-glucoside. For the metabolites mentioned above, $\mathrm{T}_{\max }$ varied between 66 and $120 \mathrm{~min}$, with $\mathrm{C}_{\max }$ concentrations between 11 and $272 \mathrm{ng} / \mathrm{mL}$. An investigation by Felgines et al. [47] and Tian et al. [161] confirmed the presence of cyanidin monoglucuronide and the low urinary excretion of native cyanidins such as cyanidin-3-rutinoside, cyanidin-3-glucoside, cyaniding sambubioside, and cyanidin-3-xylosylrutinoside.

García-Muñoz et al. [64, 65] studied ellagitannin metabolism in urine after the ingestion of $250 \mathrm{~mL}$ 
blackberry juice. It is known that the composition in ellagitannins and ellagic acid derivatives is very similar for blackberry and raspberry, as for all Rubus fruits [68]. The primary ellagitannin derivatives found in human urine were urolithin A glucuronide and urolithin B glucuronide, followed by urolithin $\mathrm{C}$ glucuronide, urolithin D glucuronide, urolithins A, B, C, D, M5, methyl urolithin A, ellagic acid, ellagic acid dimethyl ether glucuronide, ellagic acid dimethyl ether, and hexahydroxydiphenyl. However, the urolithin metabolite profiles varied considerably between volunteers, as already reported for other dietary sources of ellagitannins, as well as by Kresty et al. [89], who reported that urolithin A glucuronide and urolithin A-sulfate were detected in $85 \%$ and $60 \%$ of patients, respectively, following 12 and 26 weeks of lyophilized blackberry consumption. The fact that individuals do not respond in the same way after the ingestion of the same food complicates the interpretation of a measured value in terms of dose-response relationships.

Again, more studies in humans are required in order to identify the most specific metabolites for blackberry and better describe their kinetics in plasma and urine. Based on the aforementioned studies, it is difficult to speculate about which metabolites may be potential biomarkers of blackberry intake, while combination of urolithin metabolites in 24-h urine with cyanidin metabolites could only be a rough indicative marker for blackberry consumption, for the reasons already discussed for the other berries.

Given the high similarity of their qualitative composition, the Rubus berries could be evaluated as a combined group. A distinctive feature of the ellagitannins in the Rubus fruit is that they contain the sanguisorboyl group, a relatively uncommon ester between a galloyl group and a (S)-hexahydroxydiphenoyl moiety, which is positional isomeric as compared with the more widespread valoneoyl ester group. This building block is present at a concentration close to 50\% that of the ellagic acid [173]. Unfortunately, we are currently lacking data about the fate of this structure upon ingestion.

\section{Berries considered as one food type}

As showed above for various berry types, it is not easy to propose candidate BFIs. In most cases, berry-derived metabolites failed the specificity criterion. On the other hand, berries could be considered as a food group, and a panel of metabolites could indicate the consumption of berries without specification of the berry species. In such case, urolithins and anthocyanin metabolites, when present simultaneously in biological fluids, appear as the most relevant candidates. However, several strong limitations exist. One is the high individual variation in the production of urolithins, described with the three metabotypes A, B, and
$0[63,162,163]$. A way to overcome this issue would be to wisely sum the concentrations of the major urolithins reported in the different metabotypes: urolithin A, urolithin $\mathrm{B}$, urolithin $\mathrm{C}$, and urolithin $\mathrm{D}$, together with their glucuronide and sulfate conjugates [163]. Urolithins are produced by the opening and decarboxylation of one of the lactone rings of ellagic acid and the sequential removal of hydroxyl groups. The decarboxylation step leads first to formation of urolithin M-5, and from this urolithin D, urolithin M-6 through dehydroxylation at different positions. Further dihydroxylation lead to formation of three hydroxyurolithins: urolithin C, urolithin M-7, and two hydroxyurolithins: urolithin A and isourolithin A. Finally the monohydroxy urolithins are urolithin B and isourolithin B [45]. A number of studies investigating metabolism of ellagitannins reported urolithin A and B glucuronide and sulfate, as the main metabolites in urine and plasma, while urolithin $C$ and isourolithin A glucuronide were minor metabolites [28, $65,72,92,140,165]$. Considering anthocyanins, the following metabolites were the most frequently found in urine after different berry consumption: cyanidin glycosides, cyanidin glucuronide, malvidin glycosides, malvidin glucuronide, pelargonidin glycosides, and pelargonidin glucuronide. Delphinidin glycosides and glucuronide together with petunidin metabolites could be considered as minor metabolites. An appropriate collection of samples for both anthocyanins and urolithins should be carefully evaluated and may be difficult to implement, as these compounds have markedly different pharmacokinetics. Urolithins appear in plasma and urine for only several hours $(5 \mathrm{~h}$ and more) after an ellagitannin-containing meal, and are still detectable around $12-36 \mathrm{~h}$ [30], while anthocyanins have a $\mathrm{T}_{\max }$ of $1-2 \mathrm{~h}$ [30]. Then intensive investigations are required to verify whether such multi-metabolite panel could accurately indicate consumption of berries in large populations. Another important issue to consider is the presence of confounding factors such as consumption of other foods rich in ellagitanins and anthocyanins. Special attention should be put on nut intake, as these are a wellknown source of ellagitannins [58]. Regarding anthocyanin intake, among vegetables and fruits, red radish, red cabbage, black beans, eggplants, grapes, pomegranate, and cherries represent likely confounding factors [104].

\section{Grapes}

Among the studies selected for this review, only a few applied an untargeted metabolomics approach to obtain a holistic view of the metabolites associated with the intake of grape or grape extracts [66, 85, 168]. GarciaPerez et al. aimed to identify BFI and proposed a pipeline method using grape as an example. The method was based on the combination of an untargeted discovery approach in an acute study followed by a hypothesisdriven targeted analysis in a short-term dietary 
intervention. Using NMR, the authors first identified tartaric acid as the most discriminating metabolite in urine after red grape intake, while in a second step a targeted approach was used for quantification of this acid in an independent dietary intervention (Table 1). In the discovery study, the authors observed that other NMR signals from glucose, hippurate, and 4-hydroxyhippurate were also associated with grape intake; however, only tartaric acid increased proportionally in all participants with incremental grape intake over the duration of the study. Then they confirmed with a targeted analysis that tartaric acid excretion was elevated in 24-h urine samples after 4 days of consumption of grapes, reaching a level accounting for $19 \%$ of the dose ingested from grapes. The relationship was dose-dependent as after consumption of 50,100 , and $150 \mathrm{~g}$ of grapes, an average amount of $0.16,0.30$, and $0.49 \mathrm{mmol}$ of tartaric acid, respectively, was excreted in 24-h urine [66]. This observation complemented the results previously published by Lord et al. [97] which showed an increase in urinary tartrate excretion from 7.4 to $282 \mu \mathrm{g} / \mathrm{mg}$ of creatinine after an acute intake of $280 \mathrm{~mL}(10 \mathrm{oz})$ of grape juice and by

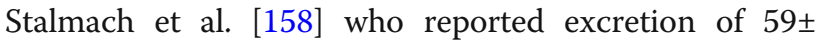
$14 \mu \mathrm{mol}$ over $24 \mathrm{~h}$ after intake of $350 \mathrm{~mL}$ of Concord grape. Some pharmacokinetics data are available for tartaric acid, showing that its excretion peaks between 4 and $8 \mathrm{~h}$ after grape intake, with a majority of the excretion occurring in the first $12 \mathrm{~h}$ [66].

Two other studies applied an untargeted metabolomics approach providing information on the changes occurring in 24-h urine fingerprints after an intervention with grape extracts for 2 or 4 weeks. In the first study on 58 subjects [168], authors showed differences in 18 metabolites detected by gas chromatography-mass spectrometry (GC-MS), corresponding to metabolites derived from host and gut microbiota metabolism of grape phenolic acids (Table 2). The other study was developed on 31 subjects after an acute or a 15-day supplementation with a drink containing grape skin extract [85]. The authors pointed out that the postprandial urinary metabolome determined by LC-MS after a single dose was characterized by phase II metabolites of polyphenols present in grape skin, whereas in 24-h urine samples collected after a prolonged consumption, phase II metabolites derived from microbial metabolism of flavanols were predominating (Table 2). However, the identified metabolites do not have a high specificity for grape consumption, since they were also detected in urine after consumption of other polyphenol-rich sources, such as cocoa, tea, red wine, or almond [144].

In term of validation of tartaric acid as BFI, the plausibility criterion seems fulfilled, as tartaric acid was described as the main organic acid in red and green grapes [66], as well as in wine [133]. Tartaric acid biosynthesis begins with L-ascorbic acid through the cleavage of a six-carbon intermediate between position $\mathrm{C} 4 / \mathrm{C} 5$. This is the predominate pathway in the Vitaceae plants [35, 146]. On the other hand, it can be present in some processed foods, such as mixed dishes $(0.42 \mathrm{~g} / \mathrm{kg})$, biscuits $(0.12-0.13 \mathrm{~g} / \mathrm{kg})$ or chocolate $(0.00-0.18 \mathrm{~g} / \mathrm{kg})$, where it is added as an acidifying agent. However, concentrations reported in these dietary sources were markedly lower than in grapes $(6-9 \mathrm{~g} / \mathrm{kg})$ and wine $[11,155]$. Actually, tartaric acid has also been reported in urine after wine intake [132, 169]. Consequently, tartaric acid could be considered as a candidate biomarker, not just for grapes but also for all grape-derived products including wine. The analytical performance, as well as chemical stability were proved in both targeted and untargeted experiments. Dose-response experiments confirm this validation criterion, while time-response for multiple doses and robustness in observational studies still have to be evaluated; see Fig. 2.

Beside tartaric acid, resveratrol metabolites can be considered as relevant candidate BFI for grape. Resveratrol is a natural stilbene mainly present in grapes and its derivatives including juices and wine. Being mainly extracted from the skins, where it is present both in the free forms ( $\underline{\text { is }}$ and $\underline{\text { trans }}$ ) and as their 3-glucosides (piceid) [101], resveratrol presence is higher in whole grapes and in red wines than in white wines and juices. Rotches-Ribalta et al. [143] provided a comprehensive overview of the resveratrol metabolic profile. The metabolism of resveratrol in humans involves the formation of glucuronides and sulfate conjugates, as well as conjugates of dihydroresveratrol (DHR), which is a metabolite derived from the hydrogenation of resveratrol by the action of the gut microbiota. Four studies showed increased levels of resveratrol and its derived metabolites after grape juice or grape extract consumption, mainly through acute studies [107, 121, 142, 143] (Table 1). Meng et al. [107] were not able to detect resveratrol in hydrolysed urine samples after the intake of 200 and $400 \mathrm{~mL}$ of grape juice, but its level was increased after doses of 600 and $1200 \mathrm{~mL}$, which suggests a dose-response relationship. Some of these studies provided pharmacokinetics data for resveratrol after grape intake. In one of them, after consumption of $1 \mathrm{~L}$ of grape juice, plasma concentrations of cis- and trans-resveratrol reached maximal levels $(36.5 \mathrm{ng} / \mathrm{mL}$ in hydrolysed samples, $0.16 \mu \mathrm{M}) 4 \mathrm{~h}$ after grape juice. Then their levels gradually decreased throughout the next $12 \mathrm{~h}$ and were no longer detectable in some subjects after $24 \mathrm{~h}$ [121]. Rotches-Ribalta et al. [142] also observed in nonhydrolysed samples that although resveratrol glucosides (piceid) can be detected in plasma as intact forms, they reached maximum concentrations $(4.5 \mathrm{ng} / \mathrm{mL}) 1 \mathrm{~h}$ after an intake of grape extract tablets, whereas after about 4 
h, much higher plasma concentrations (14.83-35.23 ng/ $\mathrm{mL}$ ) were observed for resveratrol glucuronides and after 7-8 h, even higher concentrations for DHR glucuronides $(23.06-85.70 \mathrm{ng} / \mathrm{mL})$. Plasma levels of resveratrol glucuronides showed two maximum peaks (at $4 \mathrm{~h}$ and $8 \mathrm{~h}$ ), suggesting an enterohepatic recycling [142]. Whereas only glucuronide conjugates were found in plasma samples; in urine samples, both glucuronide and sulfate conjugates were detected [142, 143]. In parallel with the plasma results, the highest excretions of DHR metabolites were achieved later than resveratrol phase II metabolites and up to $24 \mathrm{~h}$ after the intake of grape extract $[142,143]$. On the other hand, Ortuño et al. [121] observed that the urinary excretion of resveratrol isomers increased after the consumption of grape juice, reaching a peak at $0-4 \mathrm{~h}$ with a recovery of $14.2 \pm 5.9 \%$ of the dose $(0.6 \pm 0.3 \mu \mathrm{mol})$. Surprisingly, the microbial metabolite DHR was not detected in urine after grape intake, although in the same study, an increase of its urinary excretion was observed after wine consumption. It is important to keep in mind that a high variability in the amounts of resveratrol excreted in urine was observed within subjects, and this variability was even more important for microbial metabolites [142, 143]. In addition, the proportion of the various resveratrol metabolites may depend on the dose, with a higher proportion of sulfates compared to glucuronides when the ingested dose increases [142]. These aspects must be considered when formulating a quantitative threshold from which we could confirm that a person has ingested grape or not.

As regard to BFI validation (Fig. 2), the first plausibility criterion for resveratrol is fulfilled, as wines and grapes are the major dietary sources [181], although it is also present in other foods, such as apple, peanuts, pistachios, or berries. According to Farneti et al. [46], apples can be a possible source of dietary intake of resveratrol; however, resveratrol or its metabolites were not found so far in biological fluids after apple intake. Regarding the analytical performance for determining resveratrol metabolites in biological samples, Urpi-Sarda et al. [167] reported that resveratrol and its metabolites were stable in urine samples from subjects who consumed red wine under different storage and sample handling conditions, after freeze and thaw cycles and short- and long-term (5 years) storages. In parallel, quantification methods of these metabolites in biofluids showed good analytical validation parameters (ie., analytical variability, accuracy, sensitivity, specificity, precision, limits of detection, and quantification) [121, 142]. In summary, the use of the sum of resveratrol metabolites as BFI of grape might be suitable because of the significant increase after grape consumption, the specificity for this food and a known kinetics during the 24-h following the ingestion.
However, it would be highly recommended to develop further studies to evaluate more in depth the dose-response relationship and the inter-individual variation, especially for microbial metabolites. This will help to define a specific cutoff value or calibration curve to determine consumption levels, as it has been proposed for wine [182]. As the concentrations of resveratrol metabolites are rather low even after high doses, the minimum intake level measurable will have to be determined.

Except for tartaric acid and resveratrol, no other promising candidate BFI were retained for grapes. Many other studies with a targeted approach focused on the non-specific major polyphenols of grape, namely anthocyanins $[15,55,157]$, catechins, and procyanidins [99, $149,150,157]$. Other non-specific small phenolics, including a range of hydroxybenzoic acids (gallic acid, dihydroxybenzoic acid, vanillic acid, hydroxyhippuric acid, etc.), pyrogallol metabolites, hydroxyphenylacetic acids, or hydroxyphenylpropanoic acids $[158,177]$ which are either present in the grape or originate from gutmicrobiota metabolism have been reported in biofluids after grape intake. The list of these non-specific metabolites is reported in Table 2.

Among anthocyanidins, cyanidin, delphinidin, malvidin, peonidin, petunidin $[19,55,157]$, and their glucuronide conjugates [157] were identified in urine, plasma and/or ileal fluid after an acute intake of red grape juice (Table 2). However, anthocyanins are quite unstable compounds, and low bioavailability rates were found in all studies, with maximum detected levels found in the first hour after the intake of the test product and a very rapid elimination. Additionally, these metabolites have been reported after the intake of other anthocyanin-rich foods, such as berries $[15,19,55,157]$. All of these aspects obviously limit the usefulness of the anthocyaninmetabolites as biomarkers of grape intake, see Table 2.

Increased levels of a wide range of flavan-3-ols metabolites have also been observed (Table 2). Vinson et al. [171] and Lutz et al. [99] reported increased levels of epicatechin and catechin, determined in hydrolysed plasma and urine samples after intake of red grape seed extract and grape juice, respectively. Other reported metabolites are phase II conjugated forms of monomeric flavan-3-ols [157], as well as microbial-derived metabolites [158]. Sano et al. [149] detected procyanidin B1 in its intact form at low levels (nmol range) in human serum $2 \mathrm{~h}$ after intake of grape seed extract. However, as previously pointed out, these metabolites do not have enough specificity when evaluated individually since they can also increase after the intake of other flavan-3-ol food sources.

Another group of metabolites widely reported in the reviewed studies is that related to gallic acid. Some authors reported an increased excretion of this hydroxybenzoic acid 
$[79,99,157]$ or its metabolites methyl-gallic acid [131, 177] and pyrogallol [131, 158, 168] after grape consumption. Gallic acid can originate from the food itself or from the degradation of precursor compounds, in particular from the cleavage of galloylated monomeric flavan-3-ols by microbial catabolism (Monagas et al. [111]). Therefore, even if gallic acid metabolites are probably systematically present in biofluids after grape intake, they do not appear as valuable candidate BFIs for grape, if used alone, due to the major confounding of tea for example. Increased levels of quercetin and related-metabolites have also been reported in different studies after grape intake [24, 29, 33, 107], but these metabolites also increase after the intake of onions and tea, among other foods [144].

In light of these results, we can conclude that both tartaric acid and the sum of resveratrol metabolites, understood as glucuronide and sulfate conjugates, appear as the best candidate BFIs available for grapes. However, they could also reflect wine consumption. In this regard, Vázquez-Fresno et al. [169] proposed to use the combination of tartaric acid and ethyl glucuronide to monitor wine consumption. Therefore, this "tartrate-ethyl glucuronide" model could be implemented to differentiate grape intake from wine consumption.

\section{Conclusion}

The results yielded by the bibliographic survey on grape show the complexity lying on finding a unique biomarker of grape intake. Although many metabolites are strongly related to the consumption of this fruit, their variable presence in the matrix, their insufficient specificity and low bioavailability after the ingestion can limit their usefulness as a reliable biomarker of intake. Here, we suggest that the metabolites that could overcome these limitations are tartaric acid and the sum of resveratrol metabolites, associated with the non-detection of ethyl glucuronide or another alcohol biomarker as a confirmation that the detection of tartaric acid and resveratrol metabolites is due to a consumption of grapes as fruit and not as wine.

With regards to berries, considering the metabolites found in biological fluids and reported in this review, no metabolite can be suggested as a specific BFI for any particular berry. Berries share some phytochemicals such as anthocyanins and ellagitannins with other fruits or nuts, and therefore, such metabolites fail the specificity criterion-the first to be verified across the BFI validation system.

Regarding single berry types, a multi-metabolite biomarker panel could be proposed, to evaluate whether a combination of more than one metabolite has a sufficient predictive capacity. In such a way, urolithins derived from ellagic acid and ellagitannins, especially important for strawberry (and Rubus) fruits, together with pelargonidin metabolites and specific aroma compounds such as furaneol/ mesifurane conjugated to sulfate or glucuronides moieties could be associated with strawberry intake. Correspondingly, urolithins together with raspberry ketone metabolites could be associated with raspberry intake. The human metabolism of aroma compounds, especially furaneol, mesofurane for strawberries, or raspberry ketones for raspberries require more investigations regarding dose-response relationship, quantification levels, and possible interindividual variability. Profiling of volatile food compounds is gaining more and more interest, not only in field of food quality and food safety, and could be of relevance for the discovery of novel specific BFIs for berries. A tentative proposal of multimetabolite panels reflecting specific berry intake is shown in Fig. 3. It is clear that all of them require additional analysis and validation before they can be considered applicable BFIs for berries.

Of the 104 studies reported in this review, only seven applied an untargeted metabolomics approach, and fewer had the objective to identify specific BFIs for berries. Despite the fact that considerable progress has been made in the understanding of the metabolism of berry phytochemicals in humans, there are still important gaps in our knowledge on the ADME in humans of components other than anthocyanins and ellagitannins. In particular, aroma compounds definitely require further attention. Untargeted metabolomics in controlled intervention studies will be an approach of choice for making progress in this field, provided that important efforts are devoted to identify the discriminating metabolites. Metabolite identification remains the bottleneck of biomarker discovery studies applying metabolomics. A profound knowledge of the detailed composition of foods and food products is absolutely required to find a link between metabolites circulating in biological fluids and the food constituents from which they derive. Furthermore, the use of bioinformatics tools BioTransformer (http:// biotransformer.ca/) [38], Meteor Nexus (Lhasa Limited, UK), or ADMET Predictor (Simulation Plus, Lancaster, CA, USA) for in silico prediction of metabolism and for supporting the interpretation of analytical data can greatly facilitate that arduous step of metabolite identification.

Finally, as it becomes more and more evident than specific simple biomarkers will not be found for every type of food, complex modelization systems will have to be developed to allow the correct interpretation of combinations of multimetabolites biomarkers, taking into account the possible intake of confounders. 


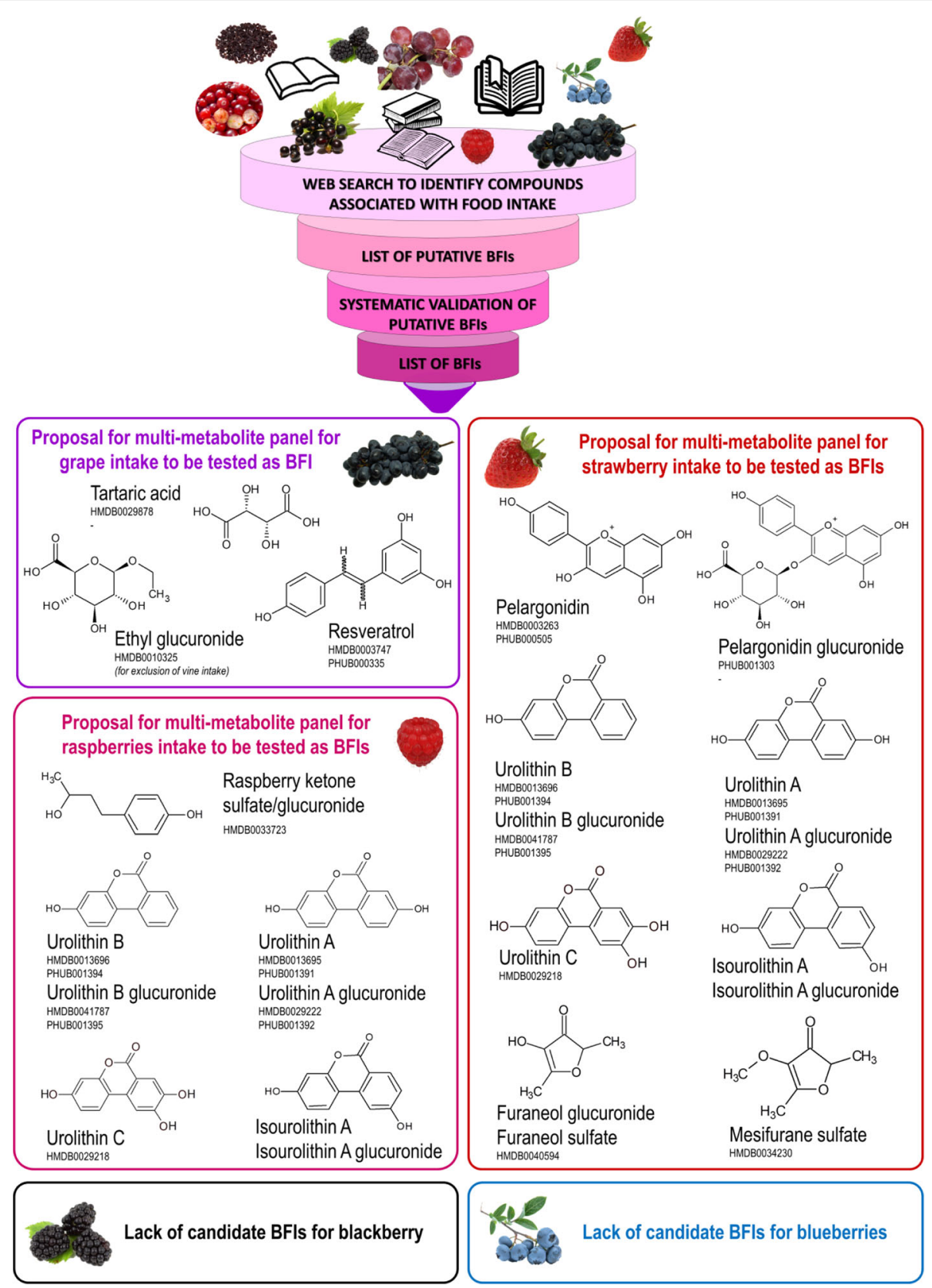

Fig. 3 Scheme of literature evaluation process for grapes and berries with BFls and potential indicative metabolites

\section{Supplementary information}

Supplementary information accompanies this paper at https://doi.org/10. 1186/s12263-020-00675-z.

Additional file 1.

\section{Authors' contributions}

This manuscript was drafted by MU, MGA, and CM. All other authors critically commented the manuscript. The authors read and approved the final manuscript.

\section{Funding}

This study was financially supported as a part of the Food Biomarkers Alliance (FoodBall) project and the FoodPhyt project, funded by National Research Councils under the umbrella of the Joint Programming Initiative a Healthy Diet for a Healthy Life (JPI HDHL), with grants from the Italian national funding organization, Ministry of Education, University and Research, MIUR (decreto n.2075 of 18/09/2015) to MU and FM; from the French Agence Nationale de la Recherche (\#ANR-14-HDHL-0002-02 and ANR-19$\mathrm{HDH} 2-0002)$ to NVM and $\mathrm{CM}$; a grant from the French Agence Nationale de la Recherche ANR-19-HDH2-0002-01 to CM; a grant from the Ministry of Economy and Competitiveness (MINECO) (PCIN-2014-133-MINECO Spain); an award from the Generalitat de Catalunya's Agency AGAUR (2017SGR1566); and funds from CIBERFES (co-funded by the FEDER Program from EU) to MGA and RL. 


\section{Availability of data and materials}

Not applicable

\section{Ethics approval and consent to participate}

Not applicable

\section{Consent for publication}

Not applicable

\section{Competing interests}

The authors declare that they have no competing interests.

\section{Author details}

${ }^{1}$ Fondazione Edmund Mach, Research and Innovation Centre Food Quality and Nutrition, Via Mach 1, 38010 San Michele all'Adige, Italy. ${ }^{2}$ Center for Omics Sciences, Proteomics and Metabolomics Facility - ProMeFa, IRCCS San Raffaele Scientific Institute, Milan, Italy. ${ }^{3}$ Biomarkers and Nutrimetabolomic Laboratory, Department of Nutrition, Food Sciences and Gastronomy, Food Technology Reference Net (XaRTA), Nutrition and Food Safety Research Institute (INSA-UB), Faculty of Pharmacy and Food Sciences, University of Barcelona, Barcelona, Spain. ${ }^{4}$ CIBER de Fragilidad y Envejecimiento Saludable (CIBERFES), Instituto de Salud Carlos III, Barcelona, Spain. ${ }^{5}$ Université Clermont Auvergne, INRAE, UNH, F-63000, Clermont-Ferrand, France. ${ }^{6}$ Department of Nutrition, Exercise and Sports, University of Copenhagen, Copenhagen, Denmark. 'Dirección de Nutrición, Instituto Nacional de Ciencias Médicas y Nutrición Slavador Zubiran, Mexico City, Mexico. ${ }^{8}$ Department of Cellular, Computational and Integrative Biology, $\mathrm{ClBIO}$, University of Trent, Trento, Italy

\section{Received: 3 April 2020 Accepted: 20 August 2020}

\section{Published online: 23 September 2020}

\section{References}

1. Afshin A, Sur PJ, Fay KA, et al. Health effects of dietary risks in 195 countries, 1990-2017: a systematic analysis for the Global Burden of Disease Study 2017. Lancet. 2019;393:1958-72. https://doi.org/10.1016/S01406736(19)30041-8.

2. Ancillotti C, Ulaszewska M, Mattivi F, Del Bubba M. Untargeted metabolomics analytical strategy based on liquid chromatography/ electrospray ionization linear ion trap quadrupole/orbitrap mass spectrometry for discovering new polyphenol metabolites in human biofluids after acute ingestion of Vaccinium myrtillus berry supplement. J Am Soc Mass Spectrom. 2019;30:381-402. https://doi.org/10.1007/s13361018-2111-y.

3. Arevström L, Bergh C, Landberg $R$, et al. Freeze-dried bilberry (Vaccinium myrtillus) dietary supplement improves walking distance and lipids after myocardial infarction: an open-label randomized clinical trial. Nutr Res. 2019; 62:13-22. https://doi.org/10.1016/j.nutres.2018.11.008.

4. Azzini $E$, Vitaglione $P$, Intorre $F$, et al. Bioavailability of strawberry antioxidants in human subjects. Br J Nutr. 2010;104:1165-73. https://doi.org/ 10.1017/S000711451000187X.

5. Banaszewski K, Park E, Edirisinghe I, et al. A pilot study to investigate bioavailability of strawberry anthocyanins and characterize postprandial plasma polyphenols absorption patterns by Q-TOF LC/MS in humans. J Berry Res. 2013;3:113-26. https://doi.org/10.3233/JBR-130048.

6. Bardagjy AS, Hu Q, Giebler KA, et al. Effects of grape consumption on biomarkers of inflammation, endothelial function, and PBMC gene expression in obese subjects. Arch Biochem Biophys. 2018;646:145-52. https://doi.org/10.1016/j.abb.2018.04.003.

7. Baron $G$, Altomare $A$, Regazzoni $L$, et al. Profiling Vaccinium macrocarpon components and metabolites in human urine and the urine ex-vivo effect on Candida albicans adhesion and biofilm-formation. Biochem Pharmacol 2020:173:113726. https://doi.org/10.1016/j.bcp.2019.113726.

8. Basu A, Lyons TJ. Strawberries, blueberries, and cranberries in the metabolic syndrome: clinical perspectives. J Agric Food Chem. 2012;60:5687-92. https://doi.org/10.1021/jf203488k.

9. Basu A, Wilkinson M, Penugonda K, et al. Freeze-dried strawberry powder improves lipid profile and lipid peroxidation in women with metabolic syndrome: baseline and post intervention effects. Nutr J. 2009:8:1-7. https:// doi.org/10.1186/1475-2891-8-43.
10. Beattie J, Crozier A, Duthie G. Potential health benefits of berries. Curr Nutr Food Sci. 2005;1:71-86. https://doi.org/10.2174/1573401052953294.

11. Bemrah $N$, Vin $K$, Sirot V, et al. Assessment of dietary exposure to annatto (E160b), nitrites (E249-250), sulphites (E220-228) and tartaric acid (E334) in the French population: the second French total diet study. Food Addit Contam Part A. 2012;29:875-85. https://doi.org/10.1080/19440049.2012.658525.

12. Bensalem J, Dudonné S, Etchamendy N, et al. Polyphenols from grape and blueberry improve episodic memory in healthy elderly with lower level of memory performance: a bicentric double-blind, randomized, placebocontrolled clinical study. Journals Gerontol Ser A. 2019;74:996-1007. https:// doi.org/10.1093/gerona/gly166.

13. Bharat D, Cavalcanti RRM, Petersen C, et al. Blueberry metabolites attenuate lipotoxicity-induced endothelial dysfunction. Mol Nutr Food Res. 2018;62: 1700601 https://doi.org/10.1002/mnfr.201700601

14. Bialasiewicz P, Prymont-Przyminska A, Zwolinska A, et al. Addition of strawberries to the usual diet decreases resting chemiluminescence of fasting blood in healthy subjects - possible health-promoting effect of these fruits consumption. J Am Coll Nutr. 2014;33:274-87 https://doi.org/10.1080/ 07315724.2013 .870502

15. Bitsch $R$, Netzel M, Frank T, et al. Bioavailability and biokinetics of anthocyanins from red grape juice and red wine. J Biomed Biotechnol. 2004;2004:293-8 https://doi.org/10.1155/S1110724304403106.

16. Blumberg JB, Camesano TA, Cassidy A, et al. Cranberries and their bioactive constituents in human health. Adv Nutr. 2013;4:618-32 https://doi.org/10. 3945/an.113.004473.

17. Boccorh RK, Paterson A, Piggott JR. Extraction of aroma components to quantify overall sensory character in a processed blackcurrant (Ribes nigrum L.) concentrate. Flavour Fragr J. 2002;17:385-91 https://doi.org/10.1002/ffj.1111.

18. Bravo L. Polyphenols: chemistry, dietary sources, metabolism, and nutritional significance. Nutr Rev. 2009:56:317-33 https://doi.org/10.1111/j.1753-4887. 1998.tb01670.x.

19. Bub A, Watzl B, Heeb D, et al. Malvidin-3-glucoside bioavailability in humans after ingestion of red wine, dealcoholized red wine and red grape juice. Eur J Nutr. 2001;40:113-20 https://doi.org/10.1007/s003940170011.

20. Cahyana Y, Gordon MH, Gibson TM. Urinary excretion of anthocyanins following consumption of strawberry and red grape juice. Int J Vitam Nutr Res. 2019;89:29-36 https://doi.org/10.1024/0300-9831/a000546.

21. Cantos E, Espín JC, Tomás-Barberán FA. Varietal differences among the polyphenol profiles of seven table grape cultivars studied by LC-DAD-MS -MS. J Agric Food Chem. 2002;50:5691-6 https://doi.org/10.1021/jf0204102.

22. Carkeet C, Clevidence BA, Novotny JA (2008) Anthocyanin excretion by humans increases linearly with increasing strawberry dose 1. J Nutr 138: 897-902. https://doi.org/138/5/897 [pii]

23. Castello F, Costabile G, Bresciani L, et al. Bioavailability and pharmacokinetic profile of grape pomace phenolic compounds in humans. Arch Biochem Biophys. 2018;646:1-9 https://doi.org/10.1016/j.abb.2018.03.021

24. Castilla $P$, Echarri $R$, Dávalos $A$, et al. Concentrated red grape juice exerts antioxidant, hypolipidemic, and antiinflammatory effects in both hemodialysis patients and healthy subjects. Am J Clin Nutr. 2006:84:252-62 https://doi.org/10.1093/ajcn/84.1.252.

25. Cerdá B, Periago P, Espín JC, Tomás-Barberán FA. Identification of urolithin A as a metabolite produced by human colon microflora from ellagic acid and related compounds. J Agric Food Chem. 2005a:53:5571-6 https://doi.org/10. 1021/jf050384i.

26. Cerdá B, Tomás-Barberán FA, Espín JC. Metabolism of antioxidant and chemopreventive ellagitannins from strawberries, raspberries, walnuts, and oak-aged wine in humans: identification of biomarkers and individual variability. J Agric Food Chem. 2005b;53:227-35 https://doi.org/10.1021/ jf049144d.

27. Chew B, Mathison B, Kimble L, et al. Chronic consumption of a low calorie, high polyphenol cranberry beverage attenuates inflammation and improves glucoregulation and HDL cholesterol in healthy overweight humans: a randomized controlled trial. Eur J Nutr. 2019:58:1223-35 https://doi.org/10. 1007/s00394-018-1643-z

28. Cortés-Martín A, García-Villalba R, González-Sarrías A, et al. The gut microbiota urolithin metabotypes revisited: the human metabolism of ellagic acid is mainly determined by aging. Food Funct. 2018;9:4100-6 https://doi.org/10.1039/C8FO00956B.

29. Costabile G, Vitale M, Luongo D, et al. Grape pomace polyphenols improve insulin response to a standard meal in healthy individuals: a pilot study. Clin Nutr. 2019;38:2727-34 https://doi.org/10.1016/j.clnu.2018.11.028. 
30. Crozier A, Del D, Clifford MN. Molecular aspects of medicine bioavailability of dietary flavonoids and phenolic compounds. Mol Aspects Med. 2010;31: 446-67 https://doi.org/10.1016/j.mam.2010.09.007.

31. Cuparencu CS, Andersen MBS, Gürdeniz G, et al. Identification of urinary biomarkers after consumption of sea buckthorn and strawberry, by untargeted LC-MS metabolomics: a meal study in adult men. Metabolomics. 2016;12:1-20 https://doi.org/10.1007/s11306-015-0934-0.

32. Curtis PJ, van der Velpen $V$, Berends $L$, et al. Blueberries improve biomarkers of cardiometabolic function in participants with metabolic syndrome-results from a 6-month, double-blind, randomized controlled trial. Am J Clin Nutr. 2019;109:1535-45 https://doi.org/10.1093/ajcn/nqy380.

33. Dávalos A, Castilla P, Gómez-Cordovés C, Bartolomé B. Quercetin is bioavailable from a single ingestion of grape juice. Int J Food Sci Nutr. 2006; 57:391-8 https://doi.org/10.1080/09637480600858662.

34. de Mello VD, Lankinen MA, Lindström J, et al. Fasting serum hippuric acid is elevated after bilberry (Vaccinium myrtillus) consumption and associates with improvement of fasting glucose levels and insulin secretion in persons at high risk of developing type 2 diabetes. Mol Nutr Food Res. 2017;61: 1700019 https://doi.org/10.1002/mnfr.201700019.

35. DeBolt S, Cook DR, Ford CM. L-Tartaric acid synthesis from vitamin C in higher plants. Proc Natl Acad Sci. 2006;103:5608-13 https://doi.org/10.1073/ pnas.0510864103.

36. Del Bo et al. Blanching Improves Anthocyanin Absorption from Highbush Blueberry (Vaccinium corymbosum L.) Puré e in Healthy Human Volunteers: A Pilot Study. 2012. https://doi.org/10.1021/jf3021333.

37. Del Bo et al. Blanching Improves Anthocyanin Absorption from Highbush Blueberry (Vaccinium corymbosum L.) Puré e in Healthy Human Volunteers: A Pilot Study. 2012.https://doi.org/10.1021/jf3021333.

38. Djoumbou-Feunang Y, Fiamoncini J, Gil-de-la-Fuente A, et al. BioTransformer: a comprehensive computational tool for small molecule metabolism prediction and metabolite identification. J Cheminform. 2019; 11:2 https://doi.org/10.1186/s13321-018-0324-5.

39. Đorđević BS, Pljevljakušić DS, Šavikin KP, et al. Essential oil from blackcurrant buds as chemotaxonomy marker and antimicrobial agent. Chem Biodivers. 2014;11:1228-40 https://doi.org/10.1002/cbdv.201400039.

40. Dragsted LO, Gao Q, Scalbert A, et al. Validation of biomarkers of food intake_critical assessment of candidate biomarkers. Genes Nutr. 2018;13:14 https://doi.org/10.1186/s12263-018-0603-9.

41. Edirisinghe I, Banaszewski K, Cappozzo J, et al. Strawberry anthocyanin and its association with postprandial inflammation and insulin. Br J Nutr. 2011; 106:913-22 https://doi.org/10.1017/S0007114511001176.

42. EFSA SRO. Evaluation of the FoodEx, the food classification system applied to the development of the EFSA Comprehensive European Food Consumption Database. EFSA J. 2011;9:1970 https://doi.org/10.2903/j.efsa. 2011.1970.

43. Erlund I, Koli R, Alfthan G, et al. Favorable effects of berry consumption on platelet function, blood pressure, and HDL cholesterol. Am J Clin Nutr. 2008; 87:323-31 https://doi.org/10.1093/ajcn/87.2.323.

44. Erlund I, Marniemi J, Hakala P, et al. Consumption of black currants, lingonberries and bilberries increases serum quercetin concentrations. Eur J Clin Nutr. 2003;57:37-42 https://doi.org/10.1038/sj.ejcn.1601513.

45. Espín JC, Larrosa M, García-Conesa MT, Tomás-Barberán F. Biological significance of urolithins, the gut microbial ellagic acid-derived metabolites: the evidence so far. Evidence-Based Complement Altern Med. 2013;2013:115 https://doi.org/10.1155/2013/270418.

46. Farneti $B$, Masuero D, Costa F, et al. Is there room for improving the nutraceutical composition of apple? J Agric Food Chem. 2015;63:2750-9 https://doi.org/10.1021/acs.jafc.5b00291.

47. Felgines $\mathrm{C}$, Talavera S, Texier $\mathrm{O}$, et al. Blackberry anthocyanins are mainly recovered from urine as methylated and glucuronidated conjugates in humans. J Agric Food Chem. 2005;53:7721-7 https://doi.org/10.1021/ jf051092k.

48. Felgines $C$, Verine $S, R a T$, et al. Human nutrition and metabolism strawberry anthocyanins are recovered in urine as glucuro- and sulfoconjugates in humans. J Nutr. 2003;133:1296-301.

49. Feliciano RP, Boeres A, Massacessi $L$, et al. Identification and quantification of novel cranberry-derived plasma and urinary (poly)phenols. Arch Biochem Biophys. 2016a;599:31-41 https://doi.org/10.1016/j.abb.2016.01.014.

50. Feliciano RP, Istas G, Heiss C, Rodriguez-Mateos A. Plasma and urinary phenolic profiles after acute and repetitive intake of wild blueberry. Molecules. 2016b;21:14-6 https://doi.org/10.3390/molecules21091120.
51. Feliciano RP, Mills CE, Istas G, et al. Absorption, metabolism and excretion of cranberry (poly)phenols in humans: a dose response study and assessment of inter-individual variability. Nutrients. 2017;9 https://doi.org/10.3390/ nu9030268.

52. Filly A, Fabiano-Tixier A-S, Lemasson $\mathrm{Y}$, et al. Extraction of aroma compounds in blackcurrant buds by alternative solvents: theoretical and experimental solubility study. Comptes Rendus Chim. 2014;17:1268-75 https://doi.org/10.1016/j.crci.2014.03.013.

53. Fleschhut J, Kratzer F, Rechkemmer G, Kulling SE. Stability and biotransformation of various dietary anthocyanins in vitro. Eur J Nutr. 2006; 45:7-18 https://doi.org/10.1007/s00394-005-0557-8.

54. Foo LY, Lu Y, Howell AB, Vorsa N. A-type proanthocyanidin trimers from cranberry that inhibit adherence of uropathogenic $\mathrm{p}$-fimbriated Escherichia coli. J Nat Prod. 2000;63:1225-8 https://doi.org/10.1021/np000128u.

55. Frank T, Netzel M, Strass G, et al. Bioavailability of anthocyanidin-3glucosides following consumption of red wine and red grape juice. Can J Physiol Pharmacol. 2003;81:423-35 https://doi.org/10.1139/y03-038.

56. Garbacki N, Tits M, Angenot L, Damas J. Inhibitory effects of proanthocyanidins from Ribes nigrum leaves on carrageenin acute inflammatory reactions induced in rats. BMC Pharmacol. 2004;4:25 https:// doi.org/10.1186/1471-2210-4-25.

57. Garcia-Aloy M, Andres-Lacueva C. Food intake biomarkers for increasing the efficiency of dietary pattern assessment through the use of metabolomics: unforeseen research requirements for addressing current gaps. J Agric Food Chem. 2018;66:5-7 https://doi.org/10.1021/acs.jafc.7b05586.

58. Garcia-Aloy M, Hulshof PJM, Estruel-Amades S, et al. Biomarkers of food intake for nuts and vegetable oils: an extensive literature search. Genes Nutr. 2019;14:7 https://doi.org/10.1186/s12263-019-0628-8.

59. Garcia-Aloy M, Llorach R, Urpi-Sarda M, et al. Novel multimetabolite prediction of walnut consumption by a urinary biomarker model in a freeliving population: the PREDIMED Study. J Proteome Res. 2014;13:3476-83 https://doi.org/10.1021/pr500425r.

60. Garcia-Aloy M, Llorach R, Urpi-Sarda M, et al. A metabolomics-driven approach to predict cocoa product consumption by designing a multimetabolite biomarker model in free-living subjects from the PREDIMED study. Mol Nutr Food Res. 2015a;59:212-20 https://doi.org/10.1002/mnfr.201400434.

61. Garcia-Aloy M, Llorach R, Urpi-Sarda M, et al. Nutrimetabolomics fingerprinting to identify biomarkers of bread exposure in a free-living population from the PREDIMED study cohort. Metabolomics. 2015b;11:15565 https://doi.org/10.1007/s11306-014-0682-6.

62. Garcia-Aloy M, Rabassa M, Casas-Agustench $P$, et al. Novel strategies for improving dietary exposure assessment: multiple-data fusion is a more accurate measure than the traditional single-biomarker approach. Trends Food Sci Technol. 2017;69:220-9 https://doi.org/10.1016/j.tifs.2017.04.013.

63. García-Mantrana I, Calatayud M, Romo-Vaquero M, et al. Urolithin metabotypes can determine the modulation of gut microbiota in healthy individuals by tracking walnuts consumption over three days. Nutrients. 2019;11:2483 https://doi.org/10.3390/nu11102483.

64. García-Muñoz C, Hernández L, Pérez A, Vaillant F. Diversity of urinary excretion patterns of main ellagitannins' colonic metabolites after ingestion of tropical highland blackberry (Rubus adenotrichus) juice. Food Res Int. 2014a;55:161-9 https://doi.org/10.1016/j.foodres.2013.10.049.

65. García-Muñoz C, Hernández L, Pérez A, Vaillant F. Diversity of urinary excretion patterns of main ellagitannins' colonic metabolites after ingestion of tropical highland blackberry (Rubus adenotrichus) juice. Food Res Int. 2014b;55:161-9 https://doi.org/10.1016/j.foodres.2013.10.049.

66. Garcia-Perez I, Posma JM, Chambers ES, et al. An analytical pipeline for quantitative characterization of dietary intake: application to assess grape intake. J Agric Food Chem. 2016;64:2423-31 https://doi.org/10.1021/acs.jafc. 5 b05878.

67. Gasperotti M, Masuero D, Guella G, et al. Evolution of ellagitannin content and profile during fruit ripening in Fragaria spp. J Agric Food Chem. 2013; 61:8597-607 https://doi.org/10.1021/jf402706h.

68. Gasperotti M, Masuero D, Vrhovsek U, et al. Profiling and accurate quantification of Rubus ellagitannins and ellagic acid conjugates using direct UPLC-Q-TOF HDMS and HPLC-DAD analysis. J Agric Food Chem. 2010;58:4602-16 https://doi.org/10.1021/jf904543w.

69. Gil-Sánchez I, Esteban-Fernández A, González de Llano D, et al. Supplementation with grape pomace in healthy women: changes in biochemical parameters, gut microbiota and related metabolic biomarkers. J Funct Foods. 2018;45:34-46 https://doi.org/10.1016/j.jf.2018.03.031. 
70. González-Barrio R, Borges G, Mullen W, Crozier A. Bioavailability of anthocyanins and ellagitannins following consumption of raspberries by healthy humans and subjects with an ileostomy. J Agric Food Chem. 2010; 58:3933-9 https://doi.org/10.1021/jf100315d.

71. González-Flores D, Gamero E, Garrido M, et al. Urinary 6-sulfatoxymelatonin and total antioxidant capacity increase after the intake of a grape juice $\mathrm{cv}$. Tempranillo stabilized with HHP. Food Funct. 2012;3:34-9 https://doi.org/10. 1039/C1FO10146C

72. González-Sarrías A, García-Villalba R, Romo-Vaquero M, et al. Clustering according to urolithin metabotype explains the interindividual variability in the improvement of cardiovascular risk biomarkers in overweightobese individuals consuming pomegranate: a randomized clinical trial. Mol Nutr Food Res. 2017;61:1600830 https://doi.org/10.1002/mnfr. 201600830.

73. Heinonen IM, Meyer AS, Frankel EN. Antioxidant activity of berry phenolics on human low-density lipoprotein and liposome oxidation. J Agric Food Chem. 1998;46:4107-12 https://doi.org/10.1021/jf980181c.

74. Henning SM, Seeram NP, Zhang Y, et al. Strawberry consumption is associated with increased antioxidant capacity in serum. J Med Food. 2010; 13:116-22 https://doi.org/10.1089/jmf.2009.0048.

75. Hollands W, Brett GM, Dainty JR, et al. Urinary excretion of strawberry anthocyanins is dose dependent for physiological oral doses of fresh fruit. Mol Nutr Food Res. 2008a;52:1097-105 https://doi.org/10.1002/mnfr. 200700372.

76. Hollands W, Brett GM, Radreau P, et al. Processing blackcurrants dramatically reduces the content and does not enhance the urinary yield of anthocyanins in human subjects. Food Chem. 2008b;108:869-78 https://doi. org/10.1016/j.foodchem.2007.11.052.

77. Hurst SM, McGhie TK, Cooney JM, et al. Blackcurrant proanthocyanidins augment IFN-y-induced suppression of IL-4 stimulated CCL26 secretion in alveolar epithelial cells. Mol Nutr Food Res. 2010;54:S159-70 https://doi.org/ 10.1002/mnfr.200900297.

78. Istas G, Feliciano RP, Weber T, et al. Plasma urolithin metabolites correlate with improvements in endothelial function after red raspberry consumption: a double-blind randomized controlled trial. Arch Biochem Biophys. 2018;651:43-51 https://doi.org/10.1016/j.abb.2018.05.016.

79. Ito H, Gonthier M, Manach C, et al (2005) Polyphenol levels in human urine after intake of six different polyphenol-rich beverages. 500-509. https://doi. org/10.1079/BJN20051522.

80. Jin $Y$, Alimbetov D, George T, et al. A randomised trial to investigate the effects of acute consumption of a blackcurrant juice drink on markers of vascular reactivity and bioavailability of anthocyanins in human subjects. Eur J Clin Nutr. 2011;65:849-56 https://doi.org/10.1038/ejcn.2011.55.

81. Kalt W, Liu Y, McDonald JE, et al. Anthocyanin metabolites are abundant and persistent in human urine. J Agric Food Chem. 2014;62:3926-34 https:// doi.org/10.1021/jf500107j.

82. Kalt W, McDonald JE, Liu Y, Fillmore SAE. Flavonoid metabolites in human urine during blueberry anthocyanin intake. J Agric Food Chem. 2017a;65: 1582-91 https://doi.org/10.1021/acs.jafc.6b05455.

83. Kalt W, McDonald JE, Vinqvist-Tymchuk MR, et al. Human anthocyanin bioavailability: effect of intake duration and dosing. Food Funct. 2017b;8: 4563-9 https://doi.org/10.1039/C7FO01074E.

84. Kanellos PT, Kaliora AC, Tentolouris NK, et al. A pilot, randomized controlled trial to examine the health outcomes of raisin consumption in patients with diabetes. Nutrition. 2014;30:358-64 https://doi.org/10. 1016/j.nut.2013.07.020

85. Khymenets $\mathrm{O}$, Andres-Lacueva C, Urpi-Sarda M, et al. Metabolic fingerprint after acute and under sustained consumption of a functional beverage based on grape skin extract in healthy human subjects. Food Funct. 2015;6: 1288-98 https://doi.org/10.1039/C4FO00684D.

86. Kim Y, Huh I, Kim J, et al. Integration of traditional and metabolomics biomarkers identifies prognostic metabolites for predicting responsiveness to nutritional intervention against oxidative stress and inflammation. Nutrients. 2017;9:233 https://doi.org/10.3390/nu9030233.

87. Kondo M, MacKinnon SL, Craft CC, et al. Ursolic acid and its esters: occurrence in cranberries and other Vaccinium fruit and effects on matrix metalloproteinase activity in DU145 prostate tumor cells. J Sci Food Agric. 2011;91:789-96 https://doi.org/10.1002/jsfa.4330.

88. Kontiokari T. Randomised trial of cranberry-lingonberry juice and Lactobacillus GG drink for the prevention of urinary tract infections in. BMJ. 2001;322:-1571 https://doi.org/10.1136/bmj.322.7302.1571.
89. Kresty LA, Fromkes JJ, Frankel WL, et al. A phase I pilot study evaluating the beneficial effects of black raspberries in patients with Barrett's esophagus. Oncotarget. 2018;9 https://doi.org/10.18632/oncotarget.10457.

90. Krikorian R, Nash TA, Shidler MD, et al. Concord grape juice supplementation improves memory function in older adults with mild cognitive impairment. Br J Nutr. 2010;103:730-4 https://doi.org/10.1017/ S0007114509992364

91. Langer S, Kennel A, Lodge JK. The influence of juicing on the appearance of blueberry metabolites $2 \mathrm{~h}$ after consumption: a metabolite profiling approach. Br J Nutr. 2018;119:1233-44 https://doi.org/10.1017/ S0007114518000855.

92. Larrosa M, González-Sarrías A, García-Conesa MT, et al. Urolithins, ellagic acid-derived metabolites produced by human colonic microflora, exhibit estrogenic and antiestrogenic activities. J Agric Food Chem. 2006;54:161120 https://doi.org/10.1021/jf0527403.

93. Lekakis J, Rallidis LS, Andreadou I, et al. Polyphenolic compounds from red grapes acutely improve endothelial function in patients with coronary heart disease. Eur J Cardiovasc Prev Rehabil. 2005;12:596-600 https://doi.org/10. 1097/00149831-200512000-00013.

94. Liu H, Garrett TJ, Su Z, et al. UHPLC-Q-Orbitrap-HRMS-based global metabolomics reveal metabolome modifications in plasma of young women after cranberry juice consumption. J Nutr Biochem. 2017:45:67-76 https://doi.org/10.1016/j.jnutbio.2017.03.007.

95. Lloyd AJ, Favé G, Beckmann M, et al. Use of mass spectrometry fingerprinting to identify urinary metabolites after consumption of specific foods. Am J Clin Nutr. 2011;94:981-91 https://doi.org/10.3945/ajen.111. 017921.

96. Lord R, Burdette C, Bralley J. Urinary markers of yeast overgrowth. Integr Med. 2004;3:24-9.

97. Lord RS, Burdette CK, Bralley JA. Significance of urinary tartaric acid. Clin Chem. 2005;51:672-3 https://doi.org/10.1373/clinchem.2004.036368.

98. Ludwig IA, Mena P, Calani L, et al. New insights into the bioavailability of red raspberry anthocyanins and ellagitannins. Free Radic Biol Med. 2015;89: 758-69 https://doi.org/10.1016/j.freeradbiomed.2015.10.400.

99. Lutz M, Castro E, García L, Henríquez C. Bioavailability of phenolic compounds in grape juice cv. Autumn Royal. CyTA - J Food. 2014;12:48-54 https://doi.org/10.1080/19476337.2013.793213.

100. Marques C, Fernandes I, Norberto S, et al. Pharmacokinetics of blackberry anthocyanins consumed with or without ethanol: a randomized and crossover trial. Mol Nutr Food Res. 2016;60:2319-30 https://doi.org/10.1002/ mnfr.201600143.

101. Mattivi F, Reniero F, Korhammer S. Isolation, characterization, and evolution in red wine vinification of resveratrol monomers. J Agric Food Chem. 1995; 43:1820-3 https://doi.org/10.1021/jf00055a013.

102. Mattivi F, Zulian C, Nicolini G, Valenti L. Wine, biodiversity, technology, and antioxidants. Ann N Y Acad Sci. 2002;957:37-56 https://doi.org/10.1111/j. 1749-6632.2002.tb02904.x.

103. Mazza G, Kay CD, Cottrell T, Holub BJ. Absorption of anthocyanins from blueberries and serum antioxidant status in human subjects. J Agric Food Chem. 2002;50:7731-7 https://doi.org/10.1021/jf020690l.

104. Mazza G, Miniati E. Anthocyanins in fruits, vegetables, and grains: CRC Press; 1994

105. McGhie TK, Ainge GD, Barnett LE, et al. Anthocyanin glycosides from berry fruit are absorbed and excreted unmetabolized by both humans and rats. J Agric Food Chem. 2003:51:4539-48 https://doi.org/10.1021/ jf026206w.

106. McNamara RK, Kalt W, Shidler MD, et al. Cognitive response to fish oil, blueberry, and combined supplementation in older adults with subjective cognitive impairment. Neurobiol Aging. 2018;64:147-56 https://doi.org/10. 1016/j.neurobiolaging.2017.12.003.

107. Meng X, Maliakal P, Lu H, et al. Urinary and plasma levels of resveratrol and quercetin in humans, mice, and rats after ingestion of pure compounds and grape juice. J Agric Food Chem. 2004;52:935-42 https://doi.org/10.1021/ jf030582e.

108. Milbury PE, Vita J a, Blumberg JB (2010) Anthocyanins are bioavailable in humans following an acute dose of cranberry juice. J Nutr 140:1099-1104. https://doi.org/10.3945/jn.109.117168.

109. Miller NJ, Rice-Evans CA. The relative contributions of ascorbic acid and phenolic antioxidants to the total antioxidant activity of orange and apple fruit juices and blackcurrant drink. Food Chem. 1997:60:331-7 https://doi. org/10.1016/S0308-8146(96)00339-1. 
110. Miller V, Yusuf S, Chow CK, et al. Availability, affordability, and consumption of fruits and vegetables in 18 countries across income levels: findings from the Prospective Urban Rural Epidemiology (PURE) study. Lancet Glob Heal. 2016:4:e695-703 https://doi.org/10.1016/S2214-109X(16)30186-3.

111. Monagas et al. Insights into the metabolism and microbial biotransformation of dietary flavan-3-ols and the bioactivity of their metabolites. 2010. https://doi.org/10.1039/COFO00132E.

112. Mueller $D$, Jung $K$, Winter $M$, et al. Human intervention study to investigate the intestinal accessibility and bioavailability of anthocyanins from bilberries. Food Chem. 2017;231:275-86 https://doi.org/10.1016/j.foodchem.2017.03. 130.

113. Mullen W, Edwards CA, Serafini M, Crozier A. Bioavailability of pelargonidin3-O-glucoside and its metabolites in humans following the ingestion of strawberries with and without cream. J Agric Food Chem. 2008:56:713-9 https://doi.org/10.1021/jf072000p.

114. Nakamura Y, Matsumoto $H$, Morifuji M, et al. Development and validation of a liquid chromatography tandem mass spectrometry method for simultaneous determination of four anthocyanins in human plasma after black currant anthocyanins ingestion. J Agric Food Chem. 2010;58:1174-9 https://doi.org/10.1021/jf9027365.

115. Netzel M, Strass G, Janssen M, et al. Bioactive anthocyanins detected in human urine after ingestion of blackcurrant juice. J Environ Pathol Toxicol Oncol. 2001;20:89-95 https://doi.org/10.1615/JEnvironPatholToxicolOncol. v20.12.20.

116. Nile SH, Park SW. Edible berries: Bioactive components and their effect on human health. Nutrition. 2014;30:134-44 https://doi.org/10.1016/j.nut.2013. 04.007.

117. Novotny JA, Chen T-Y, Terekhov Al, et al. The effect of obesity and repeated exposure on pharmacokinetic response to grape polyphenols in humans. Mol Nutr Food Res. 2017:61:1700043 https://doi.org/10.1002/mnfr. 201700043.

118. O'Byrne DJ, Devaraj S, Grundy SM, Jialal I. Comparison of the antioxidant effects of Concord grape juice flavonoids a-tocopherol on markers of oxidative stress in healthy adults. Am J Clin Nutr. 2002;76:1367-74 https:// doi.org/10.1093/ajcn/76.6.1367.

119. Ohnishi R, Ito $H$, Kasajima N, et al. Urinary excretion of anthocyanins in humans after cranberry juice ingestion. Biosci Biotechnol Biochem. 2006;70: 1681-7 https://doi.org/10.1271/bbb.60023.

120. Olas B. Berry phenolic antioxidants - implications for human health? Front Pharmacol. 2018;9 https://doi.org/10.3389/fphar.2018.00078.

121. Ortuño J, Covas M-I, Farre M, et al. Matrix effects on the bioavailability of resveratrol in humans. Food Chem. 2010;120:1123-30 https://doi.org/10. 1016/j.foodchem.2009.11.032.

122. Paller CJ, Rudek MA, Zhou XC, et al. A phase I study of muscadine grape skin extract in men with biochemically recurrent prostate cancer: safety, tolerability, and dose determination. Prostate. 2015;75:1518-25 https://doi. org/10.1002/pros.23024.

123. Pan et al. Beneficial regulation of metabolic profiles by black raspberries in human colorectal cancer patients. 2015. https://doi.org/10.1158/1940-6207. CAPR-15-0065.

124. Pantelidis G, Vasilakakis M, Manganaris G, Diamantidis G. Antioxidant capacity, phenol, anthocyanin and ascorbic acid contents in raspberries, blackberries, red currants, gooseberries and Cornelian cherries. Food Chem. 2007;102:777-83 https://doi.org/10.1016/j.foodchem.2006.06.021.

125. Pappas E, Schaich KM. Phytochemicals of cranberries and cranberry products: characterization, potential health effects, and processing stability. Crit Rev Food Sci Nutr. 2009;49:741-81 https://doi.org/10.1080/10408390802145377.

126. Park $\mathrm{E}$, Edirisinghe I, Wei $\mathrm{H}$, et al. A dose-response evaluation of freeze-dried strawberries independent of fiber content on metabolic indices in abdominally obese individuals with insulin resistance in a randomized, single-blinded, diet-controlled crossover trial. Mol Nutr Food Res. 2016;60: 1099-109 https://doi.org/10.1002/mnfr.201500845.

127. Peron G, Sut S, Pellizzaro A, et al. The antiadhesive activity of cranberry phytocomplex studied by metabolomics: intestinal PAC-A metabolites but not intact PAC-A are identified as markers in active urines against uropathogenic Escherichia coli. Fitoterapia. 2017;122:67-75 https://doi.org/ 10.1016/j.fitote.2017.08.014.

128. Praticò G, Gao Q, Scalbert A, et al. Guidelines for Biomarker of Food Intake Reviews (BFIRev): how to conduct an extensive literature search for biomarker of food intake discovery. Genes Nutr. 2018;13:3 https://doi.org/10. 1186/s12263-018-0592-8.
129. Prymont-Przyminska A, Zwolinska A, Sarniak A, et al. Consumption of strawberries on a daily basis increases the non-urate 2,2-diphenyl-1-picrylhydrazyl (DPPH) radical scavenging activity of fasting plasma in healthy subjects. J Clin Biochem Nutr. 2014;55:48-55 https://doi.org/10.3164/jcbn.13-93.

130. Puupponen-Pimia R, Nohynek L, Meier C, et al. Antimicrobial properties of phenolic compounds from berries. J Appl Microbiol. 2001;90:494-507 https://doi.org/10.1046/j.1365-2672.2001.01271.x.

131. Ras RT, Zock PL, Zebregs YEMP, et al. Effect of polyphenol-rich grape seed extract on ambulatory blood pressure in subjects with pre- and stage I hypertension. Br J Nutr. 2013;110:2234-41 https://doi.org/10.1017/ S000711451300161X.

132. Regueiro J, Vallverdú-Queralt A, Simal-Gándara J, et al. Urinary tartaric acid as a potential biomarker for the dietary assessment of moderate wine consumption: a randomised controlled trial. Br J Nutr. 2014;111:1680-5 https://doi.org/10.1017/S0007114513004108.

133. Ribereau-Gayon P, Dubourdieu D, Doneche B, Lonvaud A. The microbiology of wine and vinifications. Inc The Chemis: John Wiley Sons; 2006.

134. Rodriguez-Mateos A, del Pino-García R, George TW, et al. Impact of processing on the bioavailability and vascular effects of blueberry (poly)phenols. Mol Nutr Food Res. 2014;58:1952-61 https://doi.org/10.1002/ mnfr.201400231.

135. Rodriguez-Mateos A, Feliciano RP, Boeres A, et al. Cranberry (poly)phenol metabolites correlate with improvements in vascular function: a doubleblind, randomized, controlled, dose-response, crossover study. Mol Nutr Food Res. 2016a;60:2130-40 https://doi.org/10.1002/mnfr.201600250.

136. Rodriguez-Mateos A, Feliciano RP, Cifuentes-Gomez T, Spencer JPE. Bioavailability of wild blueberry (poly)phenols at different levels of intake. J Berry Res. 2016b;6:137-48 https://doi.org/10.3233/JBR-160123.

137. Rodriguez-Mateos $A$, Istas $G$, Boschek $L$, et al. Circulating anthocyanin metabolites mediate vascular benefits of blueberries: insights from randomized controlled trials, metabolomics, and nutrigenomics. Journals Gerontol Ser A. 2019;74:967-76 https://doi.org/10.1093/gerona/glz047.

138. Rodriguez Lanzi C, Perdicaro DJ, Antoniolli A, et al. Phenolic metabolites in plasma and tissues of rats fed with a grape pomace extract as assessed by liquid chromatography-tandem mass spectrometry. Arch Biochem Biophys. 2018;651:28-33 https://doi.org/10.1016/j.abb.2018.05.021.

139. Röhrig T, Kirsch V, Schipp D, et al. Absorption of anthocyanin rutinosides after consumption of a blackcurrant ( Ribes nigrum L.) Extract. J Agric Food Chem. 2019;67:6792-7 https://doi.org/10.1021/acs.jafc.9b01567.

140. Romo-Vaquero M, García-Villalba R, González-Sarrías A, et al. Interindividual variability in the human metabolism of ellagic acid: Contribution of Gordonibacter to urolithin production. J Funct Foods. 2015;17:785-91 https://doi.org/10.1016/j.jf.2015.06.040.

141. Roscher $\mathrm{R}$, Koch $\mathrm{H}$, Herderich $\mathrm{M}$, et al. Identification of 2,5-dimethyl-4hydroxy-3[2H]-furanone $\beta$-d-glucuronide as the major metabolite of a strawberry flavour constituent in humans. Food Chem Toxicol. 1997;35:77782 https://doi.org/10.1016/S0278-6915(97)00055-0.

142. Rotches-Ribalta M, Andres-Lacueva C, Estruch $R$, et al. Pharmacokinetics of resveratrol metabolic profile in healthy humans after moderate consumption of red wine and grape extract tablets. Pharmacol Res. 2012;66: 375-82 https://doi.org/10.1016/j.phrs.2012.08.001.

143. Rotches-Ribalta M, Urpi-Sarda M, Martí MM, et al. Resveratrol metabolic fingerprinting after acute and chronic intakes of a functional beverage in humans. Electrophoresis. 2014;35:1637-43 https://doi.org/10.1002/elps. 201300262.

144. Rothwell JA, Urpi-Sarda M, Boto-Ordonez M, et al (2012) Phenol-Explorer 2.0: a major update of the Phenol-Explorer database integrating data on polyphenol metabolism and pharmacokinetics in humans and experimental animals. Database 2012:bas031-bas031. https://doi.org/10.1093/database/bas031.

145. Russel et al. Selective bio-availability of phenolic acids from Scottish strawberries. 2009. https://doi.org/10.1002/mnfr.200800302.

146. Saito K. Metabolism of I-threotetruronic acid by Pelargonium crispum. Phytochemistry. 1992;31:1219-22 https://doi.org/10.1016/00319422(92)80264-F.

147. Sandhu AK, Huang Y, Xiao D, et al. Pharmacokinetic characterization and bioavailability of strawberry anthocyanins relative to meal intake. J Agric Food Chem. 2016;64:4891-9 https://doi.org/10.1021/acs.jafc.6b00805.

148. Sandhu AK, Miller MG, Thangthaeng N, et al. Metabolic fate of strawberry polyphenols after chronic intake in healthy older adults. Food Funct. 2018;9: 96-106 https://doi.org/10.1039/C7F001843F. 
149. SANO A, YAMAKOSHI J, TOKUTAKE S, et al. Procyanidin B1 is detected in human serum after intake of proanthocyanidin-rich grape seed extract. Biosc Biotechnol Biochem. 2003;67:1140-3 https:/doi.org/10.1271/bbb.67.1140.

150. Sasot G, Martínez-Huélamo M, Vallverdú-Queralt $A$, et al. Identification of phenolic metabolites in human urine after the intake of a functional food made from grape extract by a high resolution LTQ-Orbitrap-MS approach. Food Res Int. 2017;100:435-44 https://doi.org/10.1016/j.foodres.2017.01.020.

151. Schwab W. Natural 4-Hydroxy-2,5-dimethyl-3(2H)-furanone (Furaneol ${ }^{\circledast}$ ). Molecules. 2013;18:6936-51 https://doi.org/10.3390/molecules18066936.

152. Shukitt-Hale B, Carey AN, Jenkins D, et al. Beneficial effects of fruit extracts on neuronal function and behavior in a rodent model of accelerated aging. Neurobiol Aging. 2007;28:1187-94 https://doi.org/10.1016/j.neurobiolaging. 2006.05.031.

153. Sobolev, Ciampa, Ingallina, et al (2019) Blueberry-based meals for obese patients with metabolic syndrome: a multidisciplinary metabolomic pilot study. Metabolites 9:138. https://doi.org/10.3390/metabo9070138.

154. Spencer JPE. The impact of fruit flavonoids on memory and cognition. Br J Nutr. 2010;104:S40-7 https://doi.org/10.1017/S0007114510003934.

155. Spiller M, Spiller GA (2001) Tartaric acid content of foods. In Dietary Fiber in Human Nutrition. Boca Raton, FL CRC Press 681.

156. Sporstø| S, Scheline RR. The metabolism of 4-(4-hydroxyphenyl)butan-2-one (raspberry ketone) in rats, guinea-pigs and rabbits. Xenobiotica. 1982;12: 249-57 https://doi.org/10.3109/00498258209052463.

157. Stalmach A, Edwards CA, Wightman JD, Crozier A. Gastrointestinal stability and bioavailability of (poly)phenolic compounds following ingestion of Concord grape juice by humans. Mol Nutr Food Res. 2012;56:497-509 https://doi.org/10.1002/mnfr.201100566.

158. Stalmach A, Edwards CA, Wightman JD, Crozier A. Colonic catabolism of dietary phenolic and polyphenolic compounds from Concord grape juice. Food Funct. 2013:4:52-62 https://doi.org/10.1039/C2FO30151B.

159. Szajdek A, Borowska EJ. Bioactive compounds and health-promoting properties of berry fruits: a review. Plant Foods Hum Nutr. 2008;63:147-56 https://doi.org/10.1007/s11130-008-0097-5.

160. Tang JS, Vissers MCM, Anderson RF, et al. Bioavailable blueberry-derived phenolic acids at physiological concentrations enhance Nrf2-regulated antioxidant responses in human vascular endothelial cells. Mol Nutr Food Res. 2018;62:1700647 https://doi.org/10.1002/mnfr.201700647.

161. Tian Q, Giusti MM, Stoner GD, Schwartz SJ. Urinary excretion of black raspberry (Rubus occidentalis) anthocyanins and their metabolites. J Agric Food Chem. 2006;54:1467-72 https://doi.org/10.1021/jf052367z

162. Tomás-Barberán FA, García-Villalba R, González-Sarrías A, et al. Ellagic acid metabolism by human gut microbiota: consistent observation of three urolithin phenotypes in intervention trials, independent of food source, age, and health status. J Agric Food Chem. 2014;62:6535-8 https://doi.org/10. 1021/jf5024615.

163. Tomás-Barberán FA, González-Sarrías A, García-Villalba R, et al. Urolithins, the rescue of "old" metabolites to understand a "new" concept: metabotypes as a nexus among phenolic metabolism, microbiota dysbiosis, and host health status. Mol Nutr Food Res. 2017;61:1500901 https://doi.org/10.1002/mnfr. 201500901.

164. Torronen R, McDougall GJ, Dobson G, et al. Fortification of blackcurrant juice with crowberry: impact on polyphenol composition, urinary phenolic metabolites, and postprandial glycemic response in healthy subjects. Funct Foods. 2012;4:746-56 https://doi.org/10.1016/j.jf.2012.05.001.

165. Truchado P, Larrosa M, García-Conesa MT, et al. Strawberry processing does not affect the production and urinary excretion of urolithins, ellagic acid metabolites, in humans. J Agric Food Chem. 2012;60:5749-54 https://doi. org/10.1021/jf203641r.

166. Ulaszewska M, Vázquez-Manjarrez N, Garcia-Aloy M, et al. Food intake biomarkers for apple, pear, and stone fruit. Genes Nutr. 2018;13:29 https:// doi.org/10.1186/s12263-018-0620-8

167. Urpi-Sarda M, Zamora-Ros R, Lamuela-Raventos R, et al. HPLC-tandem mass spectrometric method to characterize resveratrol metabolism in humans. Clin Chem. 2007:53:292-9 https://doi.org/10.1373/clinchem.2006.071936.

168. van Dorsten FA, Grün CH, van Velzen EJJ, et al. The metabolic fate of red wine and grape juice polyphenols in humans assessed by metabolomics. Mol Nutr Food Res. 2009;54:897-908 https://doi.org/10.1002/mnfr. 200900212

169. Vázquez-Fresno R, Llorach R, Urpi-Sarda M, et al. An NMR metabolomics approach reveals a combined-biomarkers model in a wine interventional trial with validation in free-living individuals of the PREDIMED study. Metabolomics. 2015;11:797-806 https://doi.org/10.1007/s11306-014-0735-x

170. Verzera A, Tripodi G, Dima G, et al. Leaf removal and wine composition of Vitis vinifera L. Cv. Nero d'Avola: the volatile aroma constituents. J Sci Food Agric. 2016;96:150-9 https://doi.org/10.1002/jsfa.7075.

171. Vinson JA, Proch J, Bose P. MegaNatural ${ }^{\circledR}$ Gold grapeseed extract: in vitro antioxidant and in vivo human supplementation studies. J Med Food. 2001; 4:17-26 https://doi.org/10.1089/10966200152053677.

172. Vrhovsek U, Guella G, Gasperotti M, et al. Clarifying the Identity of the main ellagitannin in the fruit of the strawberry, Fragaria vesca and Fragaria ananassa Duch. J Agric Food Chem. 2012;60:2507-16 https://doi.org/10. 1021/jf2052256

173. Vrhovsek U, Palchetti A, Reniero F, et al. Concentration and mean degree of polymerization of Rubus ellagitannins evaluated by optimized acid methanolysis. J Agric Food Chem. 2006;54:4469-75 https://doi.org/10.1021/ jf060404w.

174. Walsh JM, Ren X, Zampariello C, et al. Liquid chromatography with tandem mass spectrometry quantification of urinary proanthocyanin A2 dimer and its potential use as a biomarker of cranberry intake. J Sep Sci. 2016;39:342-9 https://doi.org/10.1002/jssc.201500922.

175. Wang C, Zuo Y, Vinson JA, Deng Y. Absorption and excretion of cranberryderived phenolics in humans. Food Chem. 2012;132:1420-8 https://doi.org/ 10.1016/j.foodchem.2011.11.131.

176. Wang $Y$, Singh AP, Nelson HN, et al. Urinary clearance of cranberry flavonol glycosides in humans. J Agric Food Chem. 2016;64:7931-9 https://doi.org/ 10.1021/acs.jafc.6b03611.

177. Ward NC, Croft KD, Puddey IB, Hodgson JM. Supplementation with grape seed polyphenols results in increased urinary excretion of 3hydroxyphenylpropionic acid, an important metabolite of proanthocyanidins in humans. J Agric Food Chem. 2004;52:5545-9 https:// doi.org/10.1021/jf049404r.

178. Wu X, Cao G, Prior RL. Absorption and metabolism of anthocyanins in elderly women after consumption of elderberry or blueberry. J Nutr. 2002; 132:1865-71 https://doi.org/10.1093/jn/132.7.1865.

179. Xiao D, Sandhu A, Huang $Y$, et al. The effect of dietary factors on strawberry anthocyanins oral bioavailability. Food Funct. 2017;8:3970-9 https://doi.org/ 10.1039/C7FO00885F.

180. Yang J, Xiao Y-Y. Grape phytochemicals and associated health benefits. Crit Rev Food Sci Nutr. 2013;53:1202-25 https://doi.org/10.1080/10408398.2012. 692408.

181. Zamora-Ros R, Andres-Lacueva C, Lamuela-Raventós RM, et al. Concentrations of resveratrol and derivatives in foods and estimation of dietary intake in a Spanish population: European Prospective Investigation into Cancer and Nutrition (EPIC)-Spain cohort. Br J Nutr. 2008:100:188-96 https://doi.org/10.1017/S0007114507882997

182. Zamora-Ros R, Urpí-Sardà M, Lamuela-Raventós RM, et al. Resveratrol metabolites in urine as a biomarker of wine intake in free-living subjects: the PREDIMED Study. Free Radic Biol Med. 2009;46:1562-6 https://doi.org/ 10.1016/j.freeradbiomed.2008.12.023.

183. Zhang K, Zuo Y. GC-MS determination of flavonoids and phenolic and benzoic acids in human plasma after consumption of cranberry juice. J Agric Food Chem. 2004;52:222-7 https://doi.org/10.1021/jf035073r.

184. Zhang X, Sandhu A, Edirisinghe I, Burton-Freeman B (2018) An exploratory study of red raspberry (Rubus idaeus L.) (poly)phenols/metabolites in human biological samples. Food Funct 9:806-818. https://doi.org/10.1039/ C7FO00893G.

185. Zhong S, Sandhu A, Edirisinghe I, Burton-Freeman B. Characterization of wild blueberry polyphenols bioavailability and kinetic profile in plasma over 24-h period in human subjects. Mol Nutr Food Res. 2017;61:1700405 https:// doi.org/10.1002/mnfr.201700405.

\section{Publisher's Note}

Springer Nature remains neutral with regard to jurisdictional claims in published maps and institutional affiliations. 